\title{
Stability of Black Holes and Black Branes
}

\author{
Stefan Hollands ${ }^{1 *}$ and Robert M. Wald ${ }^{2 \dagger}$ \\ ${ }^{1}$ School of Mathematics, Cardiff University, \\ Cardiff, United Kingdom \\ ${ }^{2}$ Enrico Fermi Institute and Department of Physics, \\ The University of Chicago, Chicago, IL 60637, USA
}

September 5, 2012

\begin{abstract}
We establish a new criterion for the dynamical stability of black holes in $D \geq 4$ spacetime dimensions in general relativity with respect to axisymmetric perturbations: Dynamical stability is equivalent to the positivity of the canonical energy, $\mathcal{E}$, on a subspace, $\mathcal{T}$, of linearized solutions that have vanishing linearized ADM mass, momentum, and angular momentum at infinity and satisfy certain gauge conditions at the horizon. This is shown by proving that-apart from pure gauge perturbations and perturbations towards other stationary black holes $-\mathcal{E}$ is nondegenerate on $\mathcal{T}$ and that, for axisymmetric perturbations, $\mathcal{E}$ has positive flux properties at both infinity and the horizon. We further show that $\mathcal{E}$ is related to the second order variations of mass, angular momentum, and horizon area by $\mathcal{E}=\delta^{2} M-\sum_{A} \Omega_{A} \delta^{2} J_{A}-\frac{\kappa}{8 \pi} \delta^{2} A$, thereby establishing a close connection between dynamical stability and thermodynamic stability. Thermodynamic instability of a family of black holes need not imply dynamical instability because the perturbations towards other members of the family will not, in general, have vanishing linearized ADM mass and/or angular momentum. However, we prove that for any black brane corresponding to a thermodynamically unstable black hole, sufficiently long wavelength perturbations can be found with $\mathcal{E}<0$ and vanishing linearized ADM quantities. Thus, all black branes corresponding to thermodynmically unstable black holes are dynamically unstable, as conjectured by Gubser and Mitra. We also prove that positivity of $\mathcal{E}$ on $\mathcal{T}$ is equivalent to the satisfaction of a "local Penrose inequality," thus showing that satisfaction of this local Penrose inequality is necessary and sufficient for dynamical stability. Although we restrict our considerations in this paper to vacuum general relativity, most of the results of this paper are derived using general Lagrangian and Hamiltonian methods and therefore can be straightforwardly generalized to allow for the presence of matter fields and/or to the case of an arbitrary diffeomorphism covariant gravitational action.
\end{abstract}

*HollandsS@cardiff.ac.uk

†rmwa@uchicago.edu 


\section{Contents}

1 Introduction 2

2 Canonical Energy 8

2.1 Gauge choice near the horizon . . . . . . . . . . . . . . 8

2.2 First variation formulas . . . . . . . . . . . . . . . . 12

2.3 Second variations and canonical energy . . . . . . . . . . . . . 16

2.4 Evaluation of canonical energy . . . . . . . . . . . . . . . . . 21

3 Flux of canonical energy at infinity and the horizon 25

4 Stability and instability 28

4.1 The Hilbert space $\mathcal{V}$ and the degeneracies of $\mathcal{E} \ldots \ldots \ldots . \ldots 29$

4.2 Stability and instability arguments . . . . . . . . . . . . 33

5 Proof of the Gubser-Mitra conjecture 35

6 Equivalence to the local Penrose inequality 42

A Proof of Lemma 2 44

B Proof of lemmas 3 and 4

C Strengthened version of property (3) of Proposition 5

\section{Introduction}

It is of considerable interest to determine the linear stability of black holes in $D$-dimensional general relativity. It is also of interest to determine the linear stability of the corresponding black branes in $(D+p)$-dimensions, i.e., spacetimes $\mathscr{M} \times \mathbb{T}^{p}$ with metric of the form

$$
\mathrm{d} \tilde{s}_{D+p}^{2}=\mathrm{d} s_{D}^{2}+\sum_{i=1}^{p} \mathrm{~d} z_{i}^{2} .
$$

where $\mathrm{d} s_{D}^{2}$ is a black hole metric. One can analyze this issue by writing out the linearized Einstein equation off of the black hole or black brane background spacetime. One way of establishing linear stability is to find a suitable positive definite conserved norm for perturbations. Linear instability can be established by finding a solution for which some gauge independent quantity has unbounded growth in time. However, even in the very simplest cases - such as the Schwarzschild black hole [33, 40] and the Schwarzschild black string [16] - it is highly nontrivial to carry out the decoupling of equations and the fixing of gauge needed to determine stability or instability directly from the equations of motion. It is particularly difficult to analyze stability when the background is stationary but not static. In the case of a Kerr black hole in 4-dimensions, the Teukolsky formalism [38] reduces the coupled system of linear perturbation equations to a single equation for a gauge invariant, complex variable, but there is no known formalism of comparable power 
to decouple the equations in other cases, including the higher dimensional Myers-Perry analogs of Kerr; see [8, 9, 7] and references therein. Thus, it would be extremely useful to have a stability criterion for black holes and black branes that does not require one to perform a complete analysis of the linearized perturbation equations.

For the case of black branes, a simple criterion for stability was proposed in [18, 19], based on the analogy between laws of black hole mechanics and the laws of thermodynamics. We now describe their criterion. Let us assume for simplicity that we are given a stationary, asymptotically flat black hole solution with compact event horizon cross section, in vacuum general relativity (no matter fields) in $D$ spacetime dimensions. By the rigidity theorem [21, 27, we may assume that our solution is not only stationary and asymptotically flat, but has additional commuting rotational Killing fields $\psi_{1}^{a}, \ldots, \psi_{N}^{a}$, with associated angular momenta $J_{1}, \ldots, J_{N}$, where $N \leq\left\lfloor\frac{D-1}{2}\right\rfloor$. Suppose now that we do not just have one such solution, but - as usually happens - a family of solutions parameterized by the mass $M$, and the angular momenta $J_{1}, \ldots, J_{N}$, such as the Myers-Perry family [29]. As usual, we identify the entropy as $S=\frac{1}{4} A$, where $A=A\left(M, J_{1}, \ldots, J_{N}\right)$ is the area of the horizon. Consider the Hessian matrix of $S$,

$$
\operatorname{Hess}_{S}=\left(\begin{array}{cc}
\frac{\partial^{2} S}{\partial M^{2}} & \frac{\partial^{2} S}{\partial J_{A} \partial M} \\
\frac{\partial^{2} S}{\partial M \partial J_{B}} & \frac{\partial^{2} S}{\partial J_{A} \partial J_{B}}
\end{array}\right) .
$$

Then by analogy with the thermodynamical properties of ordinary laboratory type systems, the signs of the eigenvalues of $\operatorname{Hess}_{S}$ might be expected to be related to the stability of the system: For normal laboratory-type systems we expect the system to be unstable if the Hessian has a positive eigenvalue, which would correspond to a negative heat capacity. Therefore, one might expect that negativity of the Hessian, eq. (2), would be a necessary condition for black hole stability. However, this is clearly false: For example, the 4-dimensional Schwarzschild black hole has negative heat capacity, since its area is $A=16 \pi M^{2}$, so $\partial^{2} S / \partial M^{2}>0$. However, despite its negative heat capacity, the Schwarzschild black hole is well known to be linearly stable. Thus, for black holes, thermodynamic stability with respect to perturbations to other stationary black holes cannot be necessary for dynamical stability. Nevertheless, there is no obvious counterexample to the possibility that thermodynamic stability could be necessary for black brane stability, since, in particular, the Schwarzschild black string is known to be unstable [16]. Therefore, suppose we exclude consideration of black holes and restrict attention to black brane spacetimes of the form (1), where the $z_{i}$ coordinates are periodically identified with length $l$, so that horizon cross-sections are compact and have a well defined area. The Gubser-Mitra conjecture states that any such black brane spacetime is unstable for sufficiently large $l$ if the Hessian matrix (of the original $D$-dimensional black hole family) has a positive eigenvalue. In this paper, we will show that this conjecture follows as a consequence of a more fundamental dynamical stability criterion that we shall establish.

Another simple possible stability criterion that is applicable to black holes is the "local Penrose inequality," discussed recently in [10]. We reformulate this criterion as follows: Suppose, as above, we have a family of stationary, axisymmetric black hole spacetimes with mass $M$ and angular momenta $J_{1}, \ldots, J_{N}$. Since surface gravity cannot be negative, it follows immediately from the first law of black hole mechanics that the horizon

\footnotetext{
${ }^{1}$ In the presence of matter fields, there may be additional conserved charges.
} 
area, $\bar{A}$, must be a non-decreasing function of $M$ at fixed $J_{1}, \ldots, J_{N}$. Let $g_{a b}(\lambda)$ be an arbitrary smooth one-parameter family of axisymmetric metrics with $g_{a b}(0)$ being equal to a member of this family with non-zero surface gravity. Let $B$ denote the bifurcation surface of the Killing horizon of $g_{a b}(0)$. Let $\mathcal{A}(\lambda)$ denote the area of the apparent horizon of $g_{a b}(\lambda)$ on an initial slice passing through $B$. As will be shown in subsection 2.1 below, to first order in $\lambda$ the event horizon coincides with the apparent horizon. To second order in $\lambda$, the event horizon need not coincide with the apparent horizon-assuming cosmic censorship, it cannot lie inside the apparent horizon but it may lie outside of it. Nevertheless, to second order, the only contribution to the difference between the area, $A$, of the event horizon and $\mathcal{A}$ arises from the (second order) displacement of the event horizon relative to the apparent horizon in the background metric $g_{a b}(0)$. However, in the background metric, $B$ is an extremal surface 2 . It follows that to second order, we have $A(\lambda)=\mathcal{A}(\lambda)$. Now, let $\bar{g}_{a b}(\lambda)$ be the one parameter family of metrics in the black hole family such that $\bar{M}(\lambda), \bar{J}_{1}(\lambda), \ldots, \bar{J}_{N}(\lambda)$ agrees with the corresponding ADM mass and angular momenta $M(\lambda), J_{1}(\lambda), \ldots, J_{N}(\lambda)$ of $g_{a b}(\lambda)$. Then, by the first law of black hole mechanics together with the fact that the bifurcation surface of $g_{a b}(0)$ has vanishing extrinsic curvature, we automatically have $\left.(d \mathcal{A} / d \lambda)\right|_{\lambda=0}=\left.(d \overline{\mathcal{A}} / d \lambda)\right|_{\lambda=0}$. However, suppose that $\left.\delta^{2} \mathcal{A} \equiv\left(d^{2} \mathcal{A} / d \lambda^{2}\right)\right|_{\lambda=0}>\left.\delta^{2} \overline{\mathcal{A}} \equiv\left(d^{2} \overline{\mathcal{A}} / d \lambda^{2}\right)\right|_{\lambda=0}$. Then, since horizon area is nondecreasing with time (assuming the validity of cosmic censorship), $M$ is non-increasing with time (by positivity of Bondi flux), and $J_{A}$ is conserved (by axisymmetry), we obtain a contradiction with the possibility that $g_{a b}(\lambda)$ could "settle down" at late times to a black hole solution in the original family. This strongly suggests that satisfaction of the "local Penrose inequality"

$$
\delta^{2} \mathcal{A} \leq \delta^{2} \overline{\mathcal{A}}
$$

should be a necessary condition for stability of any black hole member of a family. This argument does not suggest that satisfaction of the local Penrose inequality should be sufficient for stability with respect to axisymmetric perturbations, but evidence in favor of this conjecture has been presented in [10]. In this paper, we will prove that our more fundamental stability criterion implies that satisfaction of the local Penrose inequality is necessary and sufficient for black hole stability with respect to axisymmetric perturbations.

We now describe our main results. We consider stationary, asymptotically flat black holes in $D \geq 4$ spacetime dimensions in vacuum general relativity. In section 5 , we will also consider the corresponding black branes in $D+p$ dimensions. By the rigidity theorem [21, 27], the event horizon of the black hole must be a Killing horizon, and, if rotating, the spacetime must be axisymmetric (possibly with respect to multiple rotational planes if $D \geq 5$ ). We restrict consideration to the case where the surface gravity, $\kappa$, of the event horizon is non-vanishing. In the case of a nonrotating black hole, our results apply to arbitrary, smooth, asymptotically flat solutions of the linearized field equations, but for a rotating stationary, axisymmetric black hole, some of our results apply only to perturbations that preserve axisymmetry in the rotational planes that have nonvanishing angular velocity, $\Omega_{A}$, of the horizon. For perturbations of the black hole, we define a quantity $\mathcal{E}$ - called the canonical energy - which is quadratic in the perturbation and given by an integral over a Cauchy surface, $\Sigma$, for the exterior region. (Thus, $\Sigma$ extends from spatial

\footnotetext{
${ }^{2}$ This follows from the fact that both its outgoing and ingoing expansion vanish.
} 
infinity to the bifurcation surface, $B$, of the black hole.) We will establish the following properties of $\mathcal{E}:(1)$ Without loss of generality, certain gauge conditions can be imposed near the horizon of the black hole that, in particular, ensure that the location of the true event horizon does not change to first order. When restricted to axisymmetric perturbations with vanishing linearized linear momenta, $\delta P_{i}=0$, and vanishing linearized change of horizon area, $\delta A=0$, the quantity $\mathcal{E}$ is invariant under axisymmetric gauge transformations that satisfy these conditions at the horizon and approach arbitrary asymptotic symmetries at infinity. (2) $\mathcal{E}$ is conserved in the sense that it takes the same value if evaluated on another Cauchy surface $\Sigma^{\prime}$ extending from spatial infinity to $B$. (3) For axisymmetric perturbations $\mathcal{E}$ is related to the second order variations of mass, $M$, horizon area, $A$, and angular momentum, $J_{A}$ by ${ }^{3}$

$$
\mathcal{E}=\delta^{2} M-\frac{\kappa}{8 \pi} \delta^{2} A-\sum_{A} \Omega_{A} \delta^{2} J_{A}
$$

(4) A Hilbert space $\mathcal{V}$ can be defined whose elements are square integrable initial data that (weakly) satisfy the linearized constraints, satisfy our horizon gauge conditions and, in addition, at spatial infinity have vanishing linearized ADM mass, linear momentum, and angular momentum with respect to the rotational Killing fields of the background spacetime. There is a dense domain, $\mathcal{T}$, of smooth elements of $\mathcal{V}$ on which $\mathcal{E}$ can be defined as a quadratic form. $\mathcal{E}$ is symmetric on $\mathcal{T} \times \mathcal{T}$ and is degenerate precisely on the perturbations that are "perturbations toward stationary black holes." Thus, if we define a new space $\mathcal{T}^{\prime}$ by modding out by perturbations toward stationary black holes (which, of course, includes all pure gauge perturbations), $\mathcal{E}$ is non-degenerate. Consequently, on this space, either (a) $\mathcal{E}$ is positive definite or (b) there is a $\Psi \in \mathcal{T}^{\prime}$ such that $\mathcal{E}(\Psi, \Psi)<0$. (5) Consider a foliation by Cauchy surfaces, $\Sigma(t)$ for the exterior region such that each $\Sigma(t)$ is composed of a hypersurface, $\mathscr{S}(t)$, that extends from a cross-section $C(t)$ of future null infinity $\mathscr{I}^{+}$to a cross-section $B(t)$ of the black hole horizon $\mathscr{H}^{+}$, together with the portions of future null infinity and the future horizon that lie to the past of $C(t)$ and $B(t)$ respectively. For perturbations that preserve axisymmetry in the rotational planes that have nonvanishing angular velocity, $\Omega_{A}$, of the horizon, the canonical energy on $\Sigma(t)$ (drawn as the broken red line in the figure) can be expressed as the sum of a manifestly non-negative contribution from $\mathscr{I}^{+}$(equal to the Bondi energy flux), a manifestly nonnegative contribution from $\mathscr{H}^{+}$(given by an integral involving the square of the linearized shear), and a term $\overline{\mathcal{E}}(t)$ which differs from the canonical energy evaluated on $\mathscr{S}(t)$ only by boundary contributions from $C(t)$ and $B(t)$. Furthermore, the boundary contribution from $C(t)$ vanishes if the Bondi news vanishes on $C(t)$ and the boundary contribution from $B(t)$ vanishes if the perturbed shear of the horizon vanishes on $B(t)$.

\footnotetext{
${ }^{3}$ Although $\delta^{2} M, \delta^{2} A$, and $\delta^{2} J_{A}$ individually depend upon the second order metric perturbation, it follows from the first law of black hole mechanics that the combination appearing on the right side of (4) is independent of the choice of second order metric perturbation.

${ }^{4}$ In order to make use of the machinery of null infinity, we must restrict consideration to even dimensional spacetimes, since $\mathscr{I}$ does not exist in odd dimensions 23 . We do not believe that this is an essential restriction, i.e., we believe that our results on the positivity of flux at infinity will also hold in odd dimensions.
} 


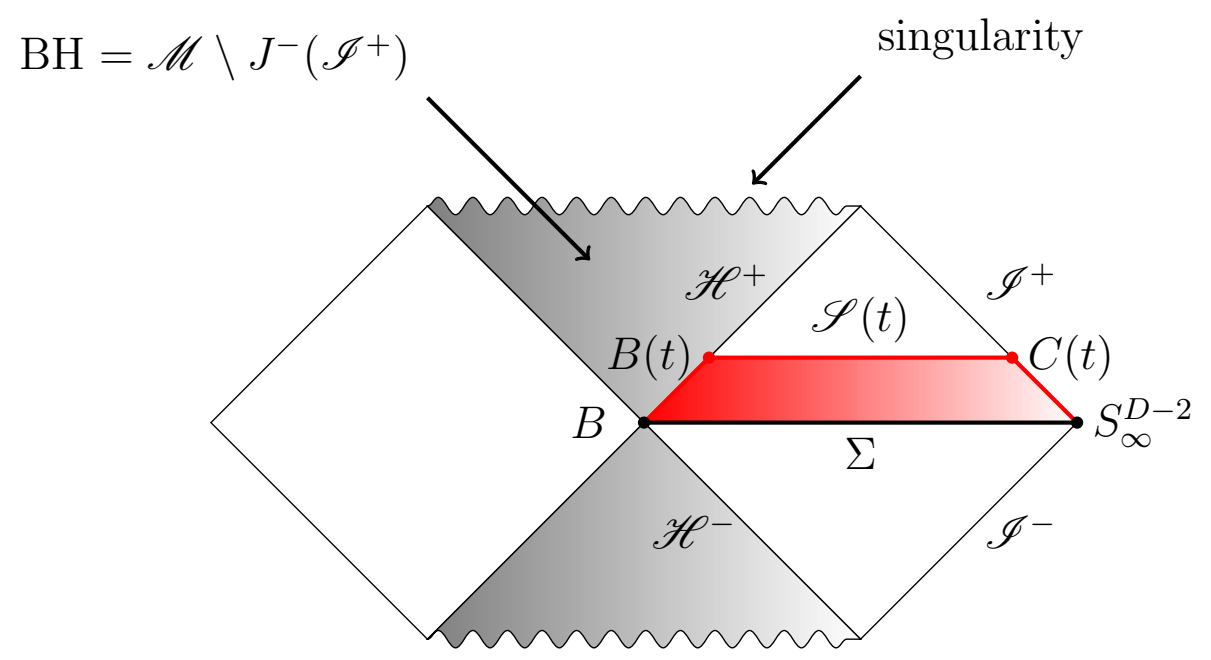

A stability criterion for black holes and black branes with respect to axisymmetric perturbations can now be formulated as follows: Suppose that $\mathcal{E}$ is positive definite on $\mathcal{T}^{\prime}$ (case (a) of point (4) above). Since $\mathcal{E}$ is conserved, we obtain a positive definite conserved norm on $\mathcal{T}^{\prime}$. Since $\mathcal{T}^{\prime}$ excludes from $\mathcal{T}$ only those perturbations that are towards stationary black holes - and stationary perturbations are automatically stable - this establishes stability of perturbations in $\mathcal{T}$, at least in the sense of establishing the nonexistence of "growing modes". Furthermore, if the black hole or black brane whose stability is being investigated is a member of a family 5 of stationary black holes/branes containing arbitrary variations of $M, J_{1}, \ldots, J_{N}$, then a general perturbation can be written as the sum of a perturbation in $\mathcal{T}$ and a perturbation to another member of the family, thereby establishing stability for general perturbations.

On the other hand, suppose $\mathcal{E}$ fails to be positive definite on $\mathcal{T}^{\prime}$ and hence, according to point (4) above, there exists a perturbation in $\mathcal{T}^{\prime}$ for which $\mathcal{E}<0$ (case (b)). We obtain a contradiction with the stability of the black hole or black brane by the following type of argument used previously in [11, 12, 13, 14]: By property (5), the quantity $\overline{\mathcal{E}}(t)$ is nonincreasing with time, and, since $\overline{\mathcal{E}}=\mathcal{E}$ on $\Sigma$, for this perturbation we have $\overline{\mathcal{E}}(t) \leq \mathcal{E}<0$. If the black hole/brane were stable, then the Bondi flux at $\mathscr{I}^{+}$and the perturbed shear at $\mathscr{H}^{+}$should go to zero at late times for this perturbation. Hence, $\overline{\mathcal{E}}(t)$ should approach the canonical energy evaluated on $\mathscr{S}(t)$. However, we have just seen that the canonical energy is bounded away from zero on $\mathscr{S}(t)$ at late times. Consequently, the perturbation cannot be approaching a solution that is a perturbation towards a stationary black hole since the linearized mass, linear momentum, and angular momentum must remain zerd 6 and $\mathcal{E}$ vanishes for perturbations towards a stationary black hole with vanishing linearized mass, linear momentum, and angular momentum. On the other hand, as already stated, any stable perturbation should become "non-radiating" at late times. However, this should be impossible, since non-stationary, non-radiating linearized perturbations should not exist.

Our argument for stability in case (a) is a genuine proof that perturbations remain bounded in a suitable norm, although it does not establish pointwise boundedness and

\footnotetext{
${ }^{5}$ If the black hole or black brane in question is not a member of a general family, one would have to separately establish the existence of a stable perturbation for given variations of $M, J_{1}, \ldots, J_{N}$.

${ }^{6}$ Mass and momentum can be radiated at quadratic order; angular momentum cannot be radiated at all for axisymmetric spacetimes.
} 
does not establish decay 7 . On the other hand, our argument for instability in case (b) has a status closer to that of a plausibility argument than a complete and rigorous proof. At the end of subsection 4.2 below, we shall indicate some possible strategies for improving upon these results.

At a conceptual level, our above criterion for black hole and black brane stability is remarkably simple: Evaluate the canonical energy $\mathcal{E}$ of initial data for perturbations that (i) satisfy the constraints, (ii) have vanishing linearized ADM mass, momentum, and angular momentum at spatial infinity, and (iii) satisfy our gauge conditions at the horizon. If $\mathcal{E} \geq 0$ for all such perturbations, then the black hole/brane is stable; if not, then it is unstable. Thus, $\mathcal{E}$ provides a "variational principle" for black hole and black brane stability, and one can test for stability by plugging in "trial initial data" into the formula for $\mathcal{E}$. Of course, the trial initial data must be chosen so as to satisfy the linearized constraints, so this variational principle cannot be used as readily as unconstrained variational principles 8 . Nevertheless, the use of our variational principle - even if it requires solving the constraints - represents an enormous simplification over performing a complete analysis of the dynamical behavior of solutions to the full set of perturbation equations.

If we have a family of black holes or black branes parameterized by $M, J_{1}, \ldots, J_{N}$, then we shall show at the beginning of section 5 that positivity of the right side of (4) for all second order variations within this family is equivalent to the negative definiteness of the Hessian (2). Thus, thermodynamic stability of the family as defined above by the Hessian criterion is equivalent to positivity of $\mathcal{E}$ for perturbations within the family. However, our criterion for dynamical stability is positivity of $\mathcal{E}$ for the case of perturbations with vanishing linearized ADM mass, momentum, and angular momentum at spatial infinity, and this excludes all perturbations within the family. Thus, for a family of black holes, the thermodynamic stability of the family provides absolutely no information about dynamical stability. In particular, this explains why the Schwarzschild black hole can be dynamically stable despite the fact that it is thermodynamically unstable: The thermodynamically unstable perturbations of Schwarzschild have a nonvanishing linearized ADM mass and thus do not "count" for the analysis of dynamical stability. However, the situation is very different for a family of black branes. If the corresponding family of black holes is thermodynamically unstable - i.e., if we can find a perturbation to another member of the black hole family that makes $\mathcal{E}<0$ - then we shall show in section 5 that one can find a sufficiently long wavelength perturbation of the black brane for which $\mathcal{E}<0$ but the linearized ADM mass, momentum, and angular momentum vanish. Consequently, the thermodynamic instability of the corresponding family of black holes implies dynamical instability of the black branes, as conjectured by Gubser and Mitra? .

In this paper, we will restrict attention to black holes (and black branes) in vacuum general relativity. However, one of the strengths of our approach is that it is based upon the Lagrangian formulation of general relativity and, hence, can be applied to nonvacuum general relativity as well as other diffeomorphism covariant theories of gravity. In

\footnotetext{
${ }^{7}$ In the stable case, the arguments of the previous paragraph strongly suggest that perturbations in $\mathcal{T}$ must decay to solutions that are perturbations towards stationary black holes.

${ }^{8}$ For the case of spherically symmetric perturbations of static, spherically symmetric spacetimes with appropriate matter fields, the linearized constraints can be solved. In that case, our variational principle (when generalized to include matter) would reduce to the unconstrained variational principle given in [35].

${ }^{9}$ Another argument in favor of the Gubser Mitra conjecture based on a "Wick rotation", applicable to certain static black branes, has previously been given by [31.
} 
particular, the notion of canonical energy can be readily generalized to these cases, and it appears likely that analogs of properties (1)-(4) above will hold. Furthermore, property (5) should hold in non-vacuum general relativity with matter satisfying appropriate energy conditions, whereas the satisfaction of property (5) in other theories of gravity should be intimately related to the positivity of energy flux at infinity and the satisfaction of the second law of black hole mechanics in these theories. Thus, it is likely that most of our stability analysis can be applied to much more general cases. However, we shall defer to a subsequent publication the analysis of black hole and black brane stability in non-vacuum general relativity and other theories of gravity.

Finally, we should emphasize that, as stated above, for rotating black holes our stability analysis is restricted to perturbations that are axisymmetric in the planes in which $\Omega_{A}$ is nonvanishing. This restriction appears to be essential since, for rotating black holes, one should easily be able to find non-axisymmetric perturbations for which $\mathcal{E}<0$ and for which the flux of canonical energy through $\mathscr{H}^{+}$(or $\mathscr{I}^{+}$if one instead uses the horizon Killing field to define $\mathcal{E}$ ) is negative. These properties should preclude the possibility of making either a stability or an instability argument of the type described above.

In the next section, we impose our horizon gauge conditions, define canonical energy, and establish many of its properties. In section 3 we prove our results on the flux of canonical energy at infinity and through the horizon. Our stability/instability arguments - including the construction of the Hilbert space $\mathcal{V}$ and the domain, $\mathcal{T}$, of $\mathcal{E}$-are given in section 4 . In section 5 , we present a proof that the thermodynamic instability of a family of black hole implies the existence of a long wavelength perturbation of the corresponding black branes with negative $\mathcal{E}$ that has vanishing linearized ADM mass, momentum, and angular momentum. This implies instability of the black brane, as conjectured by Gubser and Mitra. In section 6, we shall show that for a family of black holes, satisfaction of the local Penrose inequality (3) is equivalent to positivity of $\mathcal{E}$ on perturbations with vanishing linearized ADM mass, momentum, and angular momentum, and, consequently, for black holes, the satisfaction of the local Penrose inequality is equivalent to linear stability.

\section{Canonical Energy}

\subsection{Gauge choice near the horizon}

In this section, we consider one-parameter families of metrics $g_{a b}(\lambda)$ (not necessarily stationary) in $D \geq 4$ dimensions, that solve the field equations $G_{a b}(\lambda)=0$, are jointly smooth in the parameter $\lambda \in \mathbb{R}$ and spacetime point, and which, for $\lambda=0$, reduce to a stationary, asymptotically flat black hole spacetime $g_{a b}=g_{a b}(0)$, with bifurcate Killing horizon $\mathscr{H}$, bifurcation surface $B \subset \mathscr{H}$, and surface gravity $\kappa>0$. We also will consider corresponding families of black brane spacetimes, but the extension of our considerations to black brane spacetimes is straightforward, so we will restrict attention to black holes until section 5 .

We shall require $g_{a b}(\lambda)$ to be asymptotically flat in the sense that there exist asymptotically Minkowskian coordinates $x^{\mu}$ such that $g_{\mu \nu}(\lambda)$ differs from $\eta_{\mu \nu}$ by $O\left(\rho^{-(D-3)}\right)$ as $\rho \rightarrow \infty$-where $\rho=\left(x_{1}^{2}+\cdots+x_{D-1}^{2}\right)^{\frac{1}{2}}$ - and $N$ th derivatives of $g_{\mu \nu}(\lambda)$ are $O\left(\rho^{-(D-3+N)}\right)$. In $D=4$, we also require the Regge-Teitelboim parity conditions (see appendix A of [32]) 
to hold. In section 3, we will, in addition, impose asymptotic flatness conditions on the metric at $\mathscr{I}^{+}$.

We shall now impose certain gauge conditions on the metric near the horizon that will be used in the remainder of this paper. These conditions involve no loss of generality. Let $\mathscr{H}$ denote the black hole event horizon for the metric $g_{a b}(\lambda=0)$, and $\mathscr{H}^{ \pm}=\mathscr{H} \cap I^{ \pm}\left(\mathscr{I}^{\mp}\right)$ the future and past component. When $\lambda>0$, the surface lying at the same coordinate location as $\mathscr{H}^{+}$need not coincide with the actual event horizon of $g_{a b}(\lambda)$-indeed, there may not even be an event horizon when $\lambda>0$. It will be very useful to require that our coordinates be chosen so that, when there is a horizon for $\lambda>0$, the surface lying at the same coordinate location as $\mathscr{H}^{+}$(we could equally choose $\mathscr{H}^{-}$) continues to coincide with the actual event horizon, at least to first order in $\lambda$. Since the event horizon is a global concept and cannot easily be located exactly in any spacetime with $\lambda>0$, this might appear to be a hopelessly difficult task. However, as we shall see, the event horizon can be easily located to first order in $\lambda$, and the desired gauge condition can be imposed as follows:

First, by using Gaussian null coordinates, we can ensure that for all $\lambda$, the surface located at the same coordinates as $\mathscr{H}^{+}$is a null surface. Specifically, we may assume without loss of generality, that, near $\mathscr{H}^{+}$, each metric in our family can be written in the form

$$
g_{a b}(\lambda)=2 \nabla_{(a} u\left[\nabla_{b)} r-r^{2} \alpha(\lambda) \nabla_{b)} u-r \beta_{b)}(\lambda)\right]+\mu_{a b}(\lambda)
$$

where $r, u$ are functions defined in a neighborhood of $\mathscr{H}^{+}$that are independent of $\lambda$. The location of $\mathscr{H}^{+}$for any value of $\lambda$ is given by $r=0$, and on $\mathscr{H}^{+}, u$ is an affine parameter for any metric in our family 10 . The tensor fields $\beta_{a}, \mu_{a b}$ are orthogonal, for all $\lambda$, to the normal bundle of the $(D-2)$-dimensional surfaces $B(u, r)$, which are the joint level sets of $u, r$. That is, if

$$
n^{a}=\left(\frac{\partial}{\partial u}\right)^{a}, \quad l^{a}=\left(\frac{\partial}{\partial r}\right)^{a},
$$

then we have $\mu_{a b} n^{a}=\mu_{a b} l^{a}=\beta_{a} n^{a}=\beta_{a} l^{a}=0$. For all $\lambda$, the integral curves of $l^{a}$ are null geodesics with affine parameter $r$. For more details on Gaussian null coordinates, see e.g. [21]. We assume that $u$ is chosen such that $B \equiv B(0,0)$ is the bifurcation surface of the original metric $g_{a b}(0)$. The location of the past horizon at $\lambda=0$ is then given by $u=0$. The above form of the metric near $\mathscr{H}^{+}$is merely a choice of gauge, which we will impose for the rest of this paper. It does not use any information coming from Einstein's equation, nor any symmetry assumptions.

Although the above gauge choice requires $\mathscr{H}^{+}$to be a null surface for all $\lambda$, it does not impose the desired condition that $\mathscr{H}^{+}$coincide with the actual (future) event horizon to first order in $\lambda$. To impose this condition, we note that the future event horizon is uniquely characterized as being the outermost outgoing null surface with compact crosssections whose expansion $\vartheta$ vanishes asymptotically at late times. However, the linearized Raychaudhuri equation implies that, to first order in $\lambda$, the expansion must be constant along the horizon. Thus, the perturbed expansion $\delta \vartheta$ must vanish on the horizon at all times, and, thus, must vanish on any cross-section. Since the requirement that $\mathscr{H}^{+}$is null has already be imposed, we can ensure that $\mathscr{H}^{+}$coincides with the event horizon to

\footnotetext{
${ }^{10}$ Note that in [21, $u$ was chosen to be a Killing parameter rather than an affine parameter, resulting in some differences in the metric form in the case of a non-degenerate horizon.
} 
first order in $\lambda$ by simply imposing the requirement that its perturbed expansion vanishes on any one cross-section, such as $B$. Thus, our desired additional gauge condition is [36]

$$
\left.\delta \vartheta\right|_{B}=0 .
$$

We now wish to argue that this condition can always be imposed. Let $\phi_{s}$ be the 1parameter family of diffeomorphisms generated by the vector field $X^{a}=(1 / \sqrt{2}) f l^{a}$, where $f$ is some smooth function. Let $B_{s}=\phi_{s}[B]$ be the deformed surface, and let $\vartheta(s)$ be the expansion [of the unperturbed metric $g_{a b}=g_{a b}(0)$ ] of $B_{s}$ in the outward going future null directions $k^{a}$, normalized by $k^{a} l_{a}=1$. The vacuum Einstein equations allow one to show that the following equation holds on $B$ [1, 15]:

$$
\begin{aligned}
\frac{d}{d s} \vartheta(0) & =-D^{a} D_{a} f+\beta^{a} D_{a} f+\frac{1}{2}\left[R(\mu)-\frac{1}{2} \beta^{a} \beta_{a}+D^{a} \beta_{a}-\frac{1}{2}\left(\mathscr{L}_{n} \mu_{a b}\right) \mathscr{L}_{n} \mu^{a b}\right] f \\
& =: C(f),
\end{aligned}
$$

where $R(\mu)$ is the curvature scalar of $\mu_{a b}$, and $D_{a}$ is the derivative operator of $\mu_{a b}$. Since the background spacetime is stationary, we have $\mathscr{L}_{n} \mu_{a b}=0$ on $\mathscr{H}^{+}$. As discussed in [1], although $C$ is clearly not self-adjoint, it admits a strictly positive principal eigenfunction $\psi$. As argued in [1, 15, we must have $\lambda_{1} \geq 0$ - otherwise there would be trapped surfaces in the exterior, which is not possible. We now show that $\lambda_{1}$ is strictly positive, $\lambda_{1}>0$. (For another argument leading to the same conclusion in the more general context of "nonevolving horizons", see Prop. 3 of [26].) To do so, we use the fact that in the background metric, $B$ is the bifurcation surface of a bifurcate Killing horizon with horizon Killing field $K^{a}$. We claim first that in our Gaussian null coordinates, $K^{a}$ takes the remarkably simple form

$$
K^{a}=\kappa\left[u\left(\frac{\partial}{\partial u}\right)^{a}-r\left(\frac{\partial}{\partial r}\right)^{a}\right]
$$

where $\kappa$ is the surface gravity of the black hole. To prove this, we note that the standard relation between the affine parameter $u$ on $\mathscr{H}^{+}$and the flow parameter of the Killing field $K^{a}$ on $\mathscr{H}^{+}$implies that on $\mathscr{H}^{+}$(i.e., when $r=0$ ) we have $K^{a}=\kappa u \partial / \partial u$. It follows that on $\mathscr{H}^{+}$, flow under $K^{a}$ by parameter $\alpha$ maps $u$ to $u \exp (\kappa \alpha)$ and $n^{a}$ to $n^{a} \exp (\kappa \alpha)$. Since $l^{a}$ on $\mathscr{H}^{+}$is uniquely determined up to scale by orthogonality to $(d u)_{a}$, it follows that flow under $K^{a}$ maps $l^{a}$ to $l^{a} \exp (-\kappa \alpha)$ on $\mathscr{H}^{+}$. Since flow under $K^{a}$ maps null geodesics to null geodesics, it follows that flow under $K^{a}$ by parameter $\alpha$ acts the Gaussian null coordinates as follows throughout the domain of validity of the coordinates: $u \rightarrow u \exp (\kappa \alpha), r \rightarrow r \exp (-\kappa \alpha)$, and $x^{A} \rightarrow x^{A}$, where $x^{A}$ denotes the remaining Gaussian null coordinates. The infinitesimal form of this transformation is just (9), as we desired to show.

Now consider the above 1-parameter family of surfaces $B_{s}$ with $f=\psi$. For $s_{1}, s_{2}>$ 0 , the surface $B_{s_{2}}$ is obtained from the surface $B_{s_{1}}$ by Killing transport with Killing parameter $\frac{1}{\kappa} \log \left(s_{2} / s_{1}\right)$. Furthermore, $\tilde{k}^{a}=(1 / r) k^{a}$ is Lie-transported by $K^{a}$. It follows immediately that the expansion $\tilde{\vartheta}(s)$ with respect to $\tilde{k}^{a}$ is independent of $s$. From this, it follows that the expansion $\vartheta(s)$ with respect to $k^{a}$ is given by $\vartheta(s)=s \vartheta(1)$, where $\vartheta(1)$ denotes the expansion of the surface $B_{1}$. But we have $\frac{d}{d s} \vartheta(0)=\lambda_{1} \psi$. Consequently, if $\lambda_{1}=0$, we would have $\vartheta(1)=0$, i.e. $B_{1}$ would be a MOTS lying outside the black hole, which is impossible. This establishes that $\lambda_{1}>0$. 
Thus, we have shown that the stability operator $C$ on $B$, defined by (8)), has a strictly positive principal eigenvalue. But the principal eigenvalue of the adjoint $C^{*}$ is equal to that of $C$ (see lemma 4.1 of [1]), so $C^{*}$ cannot have a kernel. This implies that we can uniquely solve the equation $C(f)=j$ for any smooth $j$. Solving this equation with $j=-\left.\delta \vartheta\right|_{B}$, we find that the 1-parameter family of metrics $\phi_{\lambda}^{*} g_{a b}(\lambda)$ will have $\left.\vartheta\right|_{B}=0$ to (zeroth and-) first order in $\lambda$. Thus, we have proven that for any metric perturbation $\gamma_{a b}=\frac{d}{d \lambda} g_{a b}(0)$, we can apply a first order gauge transformation $\gamma_{a b} \rightarrow \gamma_{a b}+\mathscr{L}_{X} g_{a b}$ of the form $X^{a}=(1 / \sqrt{2}) f l^{a}$ that imposes eq. (17).

We are still free, without destroying our previous gauge choices, to apply a 1-parameter family of diffeomorphisms $\phi_{s}$ leaving $r, u$ invariant. Such a diffeomorphism is locally (near $\mathscr{H}^{+}$) uniquely determined by its action on the bifurcation surface $B$, and at the linearized level corresponds to a gauge vector field $\xi^{a}$ tangent to $B$ satisfying $\mathscr{L}_{\xi} r=0=\mathscr{L}_{\xi} u$. It is possible to use this freedom to impose the additional gauge condition

$$
\left.\mu^{a b} \delta \mu_{a b}\right|_{B}=\text { const. }
$$

or, equivalently,

$$
\left.\delta \epsilon\right|_{B}=\left.\frac{\delta A}{A} \epsilon\right|_{B}
$$

where $\epsilon$ denotes the background volume form on $B$ and $\delta A$ denotes the perturbed area of $B$. To prove this, we note that $\delta \mu_{a b}$ changes under a linear gauge transformation of the kind we just described by $2 D_{(a} \xi_{a)}$, where $D_{a}$ denotes the derivative operator on $B$. Thus, to impose the desired gauge, we must solve $2 D^{a} \xi_{a}=-\mu^{a b} \delta \mu_{a b}+$ const. The necessary and sufficient condition for obtaining a solution to this equation is that the right side integrates to zero over $B$, which can be achieved by an appropriate choice of the constant. It is easily seen that (10) is equivalent to (11).

We summarize our gauge choices in the following lemma:

Lemma 1. Let $\left(\mathscr{M}, g_{a b}\right)$ a stationary black hole spacetime satisfying the vacuum Einstein equations, and let $g_{a b}(\lambda)$ a 1-parameter family of solutions perturbing $g_{a b}=g_{a b}(0)$. Then near $\mathscr{H}^{+} \subset \mathscr{M}$, the metric can be brought into the form (5) with $\left.\delta \vartheta\right|_{B}=0$ (and, hence, the perturbed expansion vanishes on all of $\mathscr{H}^{+}$). Furthermore, the gauge condition (11) can be imposed at $B$.

Remark: The gauge freedom that remains on a perturbation $\gamma_{a b}$ after the imposition of the gauge conditions of lemma 1 is $\gamma_{a b} \rightarrow \gamma_{a b}+\mathscr{L}_{X} g_{a b}$ where $X^{a}$ is a smooth vector field that is an asymptotic symmetry near infinity, is tangent to $\mathscr{H}^{+}$, satisfies $\left.\mathscr{L}_{n} X^{a}\right|_{\mathscr{H}^{+}}=f n^{a}$ with $n^{a} \nabla_{a} f=0$, and satisfies $\left.\left(\mu^{a b} \nabla_{a} X_{b}\right)\right|_{B}=0$.

Finally, the black hole background metric $g_{a b}(0)$ is stationary, with Killing field $t^{a}$. By the rigidity theorem, we know that $t^{a}$ can be decomposed as

$$
t^{a}=K^{a}-\sum_{A=1}^{N} \Omega_{A} \psi_{A}{ }^{a},
$$

where, for $\lambda=0, K^{a}$ is a Killing field normal to $\mathscr{H}, \psi_{A}^{a}$ are Killing fields with closed orbits, and $\Omega_{A}$ are constants called the "angular velocities of the horizon". On the future horizon, we have from (9)

$$
\left.K^{a}\right|_{\mathscr{H}^{+}}=\kappa u\left(\frac{\partial}{\partial u}\right)^{a}
$$


In our analysis, we will later restrict attention to the axisymmetric case, although we do not make any such restriction now. More precisely, we will assume later that for each $A$ appearing in the sum in (12), there is a corresponding Killing field $\psi_{A}^{a}(\lambda)$ of $g_{a b}(\lambda)$ for $\lambda>0$. Without loss of generality, we may assume that $\psi_{A}^{a}(\lambda)=\psi_{A}^{a}$, i.e., it is compatible with the gauge choices we have made above to additionally require that our gauge is chosen so that the $\psi_{A}^{a}$ do not vary with $\lambda$. For $\lambda>0, g_{a b}(\lambda)$ is not, in general, stationary, so there are no corresponding Killing fields $t^{a}(\lambda)$ or $K^{a}(\lambda)$. Nevertheless, it will be useful to consider the $\lambda$-independent vector fields $t^{a}$ and $K^{a}$ in the spacetime $\left(\mathscr{M}, g_{a b}(\lambda)\right)$, and we will do so in the next subsection. However, we caution the reader that, in the event that $g_{a b}(\lambda)$ is a stationary black hole, with Killing fields $t^{a}(\lambda)$ and $K^{a}(\lambda)$, we may not assume that $t^{a}(\lambda)=t^{a}$ or $K^{a}(\lambda)=K^{a}$. Indeed, if $g_{a b}(\lambda)$ is a stationary black hole with surface gravity or horizon angular velocities differing from that of $g_{a b}(0)$, it would be manifestly inconsistent with eqs.(12) and (13) for $t^{a}(\lambda)=t^{a}$ and $K^{a}(\lambda)=K^{a}$. Thus, we will have to exercise care at the end of section 2.3 when identifying perturbations toward stationary black holes, since we cannot assume that these perturbations are manifestly stationary in our gauge.

\section{$2.2 \quad$ First variation formulas}

In this subsection, we will develop the machinery needed to define the canonical energy and establish its properties.

The Lagrangian for vacuum general relativity is

$$
L_{a_{1} \ldots a_{D}}=\frac{1}{16 \pi} R \epsilon_{a_{1} \ldots a_{D}}
$$

where $R$ is the Ricci scalar and $\epsilon_{a_{1} \ldots a_{D}}$ is the positively oriented 11 volume form of a $D$ dimensional spacetime with metric $g_{a b}$. We will consider the variations of $L$ and other quantities under smooth, one-parameter variations of the metric $g_{a b}(\lambda)$, and, occasionally, under two-parameter variations $g_{a b}\left(\lambda_{1}, \lambda_{2}\right)$. We will write

$$
\delta g_{a b}=\left.\frac{d}{d \lambda} g_{a b}\right|_{\lambda=0}, \quad \delta L=\left.\frac{d}{d \lambda} L\right|_{\lambda=0}, \quad \delta^{2} L=\left.\frac{d^{2}}{d \lambda^{2}} L\right|_{\lambda=0} \quad \text { etc. }
$$

for the derivative(s) at $\lambda=0$. In cases where our variational formulas hold for all $\lambda$, we will use the notation " $d / d \lambda$ " rather than " $\delta . "$

The variation of $L$ can be written as

$$
\frac{d}{d \lambda} L(g)=E(g) \cdot \frac{d}{d \lambda} g+\mathrm{d} \theta\left(g ; \frac{d}{d \lambda} g\right),
$$

where $E=0$ are the field equations 12 and $\theta$ corresponds to the boundary term that would arise if the variation were performed under an integral sign, namely

$$
\theta_{a_{1} \ldots a_{d-1}}=\frac{1}{16 \pi} v^{c} \epsilon_{c a_{1} \ldots a_{d-1}}
$$

\footnotetext{
${ }^{11} \mathrm{We}$ choose orientations in this paper is as follows: The orientation of $\mathscr{M}$ is defined by declaring the Gaussian null coordinates $\left(r, u, x^{1}, \ldots, x^{D-1}\right)$ to be right-handed. The orientation of $\Sigma$ is defined by declaring $\left(r, x^{1}, \ldots, x^{D-1}\right)$ to be left-handed, and the orientation of $B$ is defined by declaring $\left(x^{1}, \ldots, x^{D-1}\right)$ to be right-handed.

${ }^{12}$ More precisely, $E^{a b}{ }_{c_{1} \ldots c_{D}}=-\frac{1}{16 \pi} \epsilon_{c_{1} \ldots c_{D}}\left(R^{a b}-\frac{1}{2} R g^{a b}\right)$
} 
where

$$
v^{a}=g^{a c} g^{b d}\left(\nabla_{d} \frac{d}{d \lambda} g_{b c}-\nabla_{c} \frac{d}{d \lambda} g_{b d}\right) .
$$

The symplectic current $(D-1)$-form $\omega$ is defined as

$$
\omega\left(g ; \frac{\partial}{\partial \lambda_{1}} g, \frac{\partial}{\partial \lambda_{2}} g\right)=\frac{\partial}{\partial \lambda_{1}} \theta\left(g ; \frac{\partial}{\partial \lambda_{2}} g\right)-\frac{\partial}{\partial \lambda_{2}} \theta\left(g ; \frac{\partial}{\partial \lambda_{1}} g\right) .
$$

Thus, $\omega$ depends on the unperturbed metric and a pair of perturbations $\left(\frac{\partial}{\partial \lambda_{1}} g, \frac{\partial}{\partial \lambda_{2}} g\right)$, and it is antisymmetric in $\left(\frac{\partial}{\partial \lambda_{1}} g, \frac{\partial}{\partial \lambda_{2}} g\right)$. If both perturbations satisfy the linearized equations of motion, then it follows by taking a second, antisymmetrized variation of (16) that $\omega$ is closed,

$$
\mathrm{d} \omega=0 .
$$

Concretely, $\omega\left(g ; \gamma_{1}, \gamma_{2}\right)$ is given by

$$
\omega_{a_{1} \ldots a_{D-1}}=\frac{1}{16 \pi} w^{c} \epsilon_{c a_{1} \ldots a_{D-1}},
$$

where

$$
w^{a}=P^{a b c d e f}\left(\gamma_{2 b c} \nabla_{d} \gamma_{1 \text { ef }}-\gamma_{1 b c} \nabla_{d} \gamma_{2 \text { ef }}\right)
$$

and

$$
P^{a b c d e f}=g^{a e} g^{f b} g^{c d}-\frac{1}{2} g^{a d} g^{b e} g^{f c}-\frac{1}{2} g^{a b} g^{c d} g^{e f}-\frac{1}{2} g^{b c} g^{a e} g^{f d}+\frac{1}{2} g^{b c} g^{a d} g^{e f} .
$$

The symplectic form $W_{\Sigma}\left(g ; \gamma_{1}, \gamma_{2}\right)$ is obtained by integrating $\omega$ over a Cauchy surface $\Sigma$,

$$
W_{\Sigma}\left(g ; \gamma_{1}, \gamma_{2}\right) \equiv \int_{\Sigma} \omega\left(g ; \gamma_{1}, \gamma_{2}\right)
$$

In the case mostly considered in this paper-where $g_{a b}=g_{a b}(0)$ is a stationary black hole with bifurcate Killing horizon (see the previous subsection) - we take $\Sigma$ to be a Cauchy surface for the exterior region, i.e., a spacelike slice that goes from the black hole bifurcation surface $B$ to spatial infinity. For $D>4$ this integral manifestly converges for our asymptotic conditions on the metric near spatial infinity (see the second paragraph of the previous subsection). For $D=4$, the Regge-Teitelboim parity conditions assure convergence. If $\left(\gamma_{1}, \gamma_{2}\right)$ both satisfy the linearized field equations around a solution $g_{a b}$ to Einstein's equations, then then it follows from $\mathrm{d} \omega=0$ and our asymptotic conditions near spatial infinity that

$$
W_{\Sigma^{\prime}}\left(g ; \gamma_{1}, \gamma_{2}\right)=W_{\Sigma}\left(g ; \gamma_{1}, \gamma_{2}\right)
$$

where $\Sigma^{\prime}$ is another Cauchy surface extending from $B$ to spatial infinity.

We can bring the symplectic form into a more recognizable form by performing a spacetime split of the spacetime metric into the canonically conjugate variables $\left(h_{a b}, p^{a b}\right)$, where $h_{a b}$ is the induced metric on $\Sigma$ and

$$
p^{a b}=h^{\frac{1}{2}}\left(K^{a b}-h^{a b} K\right),
$$

with $K_{a b}$ the extrinsic curvature of $\Sigma$. Our asymptotic conditions near spatial infinity imply that 13

$$
h_{a b}=\delta_{a b}+O\left(\rho^{-(D-3)}\right), \quad p^{a b}=O\left(\rho^{-(D-2)}\right),
$$

\footnotetext{
${ }^{13}$ Here it is assumed that $\Sigma$ approaches a surface of constant $x^{0}$ in our asymptotically Minkowskian coordinates.
} 
with the $N$-th spatial derivatives of $h_{a b}$ and $p^{a b}$ falling off faster by a factor of $\rho^{-N}$. Note that we have followed the standard practice of incorporating the volume element of $\Sigma$ into the definition of $p^{a b}$, as is necessary in order that $\left(h_{a b}, p^{a b}\right)$ be canonically conjugate. Thus, $p^{a b}$ is a density of weight $\frac{1}{2}$. Our integrals below involving $p^{a b}$ will be taken with respect to a fixed, nondynamical (e.g., coordinate) volume element $e_{0}$ on $\Sigma$, such that the volume element $\nu^{a} \epsilon_{a b_{1} \ldots b_{D-1}}$ (where $\nu^{a}$ is the future directed unit normal to $\Sigma$ ) associated with $h_{a b}$, is equal to $h^{\frac{1}{2}}\left(e_{0}\right)_{b_{1} \ldots b_{D-1}}$. A perturbation $\delta g_{a b}$ satisfying the linearized Einstein equations corresponds to a pair $\left(\delta h_{a b}, \delta p^{a b}\right)$ satisfying the linearized constraints and the linearized Hamiltonian equations of motion. In terms of $\left(\delta h_{a b}, \delta p^{a b}\right)$ the symplectic form as defined by eq. (24) is simply 14

$$
W_{\Sigma}\left(g ; \delta_{1} g, \delta_{2} g\right)=-\frac{1}{16 \pi} \int_{\Sigma}\left(\delta_{1} h_{a b} \delta_{2} p^{a b}-\delta_{2} h_{a b} \delta_{1} p^{a b}\right) .
$$

Here, and in the following, we omit the reference volume form $e_{0}$.

For an arbitrary vector field $X^{a}$, the associated Noether current $\mathcal{J}_{X}$ is defined by

$$
\mathcal{J}_{X}(g)=\theta\left(g ; \mathscr{L}_{X} g\right)-i_{X} L(g)
$$

where $i_{X}$ denotes contraction of $X^{a}$ into the first index of a differential form such as $L_{a_{1} \ldots a_{D}}$. A simple calculation [24] shows that the first variation of $\mathcal{J}_{X}$ satisfies

$$
\frac{d}{d \lambda} \mathcal{J}_{X}=-i_{X}\left(E \cdot \frac{d}{d \lambda} g\right)+\omega\left(g ; \frac{d}{d \lambda} g, \mathscr{L}_{X} g\right)+\mathrm{d}\left[i_{X} \theta\left(g, \frac{d}{d \lambda} g\right)\right],
$$

where, in this formula, it has not been assumed that $g_{a b}$ satisfies the field equations nor that $d g_{a b} / d \lambda$ satisfies the linearized field equations. Furthermore, it can be shown [25] that $\mathcal{J}_{X}$ can be written in the form

$$
\mathcal{J}_{X}=C_{X}+\mathrm{d} Q_{X}
$$

where $C_{X} \equiv C_{a} X^{a}$ are the constraints of the theory [35] and $Q_{X}$ is the Noether charge. In the case of general relativity in vacuum, eq. (14), we have

$$
\left(C_{X}\right)_{a_{1} \ldots a_{D-1}}=\frac{1}{8 \pi} X^{a} G_{a}^{b} \epsilon_{b a_{1} \ldots a_{D-1}}
$$

and

$$
\left(Q_{X}\right)_{a_{1} \ldots a_{D-2}}=-\frac{1}{16 \pi} \nabla_{b} X_{c} \epsilon^{b c}{ }_{a_{1} \ldots a_{D-2}} .
$$

Combining eqs. (30) and (31), we obtain

$$
\omega\left(g ; \frac{d}{d \lambda} g, \mathscr{L}_{X} g\right)=i_{X}\left(E(g) \cdot \frac{d}{d \lambda} g\right)+\frac{d}{d \lambda} C_{X}(g)+\mathrm{d}\left[\frac{d}{d \lambda} Q_{X}(g)-i_{X} \theta\left(g ; \frac{d}{d \lambda} g\right)\right]
$$

It should be emphasized that eq. (34) is an identity that holds for arbitrary $X^{a}$ and $g_{a b}(\lambda)$.

We now impose the condition that our one-parameter family $g_{a b}(\lambda)$ is composed of asymptotically flat solutions to Einstein's equation, so $E=C_{a}=0$. We also require that

\footnotetext{
${ }^{14}$ The pullback of (21) to a spacelike hypersurface was computed in eq. (4.14) of [2]. There is an additional "boundary term" present in their expression for $W_{\Sigma}$, which is easily seen to vanish on account of our boundary conditions at $B$ and our asymptotic conditions at infinity.
} 
$X^{a}$ be an asymptotic symmetry near infinity, i.e., we require $\mathscr{L}_{X} g_{a b}$ to satisfy the same asymptotic conditions at infinity as $\delta g_{a b}$, so that $X^{a}$ suitably approaches a Killing field of the Minkowskian background as $\rho \rightarrow \infty$. Integrating eq. (34) over a compact region, $K$, of $\Sigma$, extending from $B$ to a surface, $S$, in the asymptotic region, we obtain, for all $\lambda$

$$
\int_{K} \omega\left(g ; \frac{d}{d \lambda} g, \mathscr{L}_{X} g\right)=\int_{S}\left[\frac{d}{d \lambda} Q_{X}(g)-i_{X} \theta\left(g ; \frac{d}{d \lambda} g\right)\right]-\int_{B}\left[\frac{d}{d \lambda} Q_{X}(g)-i_{X} \theta\left(g ; \frac{d}{d \lambda} g\right)\right] .
$$

Taking the limit as $S \rightarrow \infty$, we obtain

$$
W_{\Sigma}\left(g ; \frac{d}{d \lambda} g, \mathscr{L}_{X} g\right)=\int_{\infty}\left[\frac{d}{d \lambda} Q_{X}(g)-i_{X} \theta\left(g ; \frac{d}{d \lambda} g\right)\right]-\int_{B}\left[\frac{d}{d \lambda} Q_{X}(g)-i_{X} \theta\left(g ; \frac{d}{d \lambda} g\right)\right],
$$

where $\int_{\infty}\left[\frac{d}{d \lambda} Q_{X}(g)-i_{X} \theta\left(g ; \frac{d}{d \lambda} g\right)\right] \equiv \lim _{S \rightarrow \infty} \int_{S}\left[\frac{d}{d \lambda} Q_{X}(g)-i_{X} \theta\left(g ; \frac{d}{d \lambda} g\right)\right]$. For $D>4$ or for the case where $X$ is an asymptotic translation in $D=4$, this limit exists independently of how $S$ approaches infinity because the integral defining $W_{\Sigma}\left(g ; \frac{d}{d \lambda} g, \mathscr{L}_{X} g\right)$ converges absolutely by virtue of our assumed asymptotic conditions. For asymptotic boosts and rotations in $D=4$, the limit must be taken where $S$ approaches a two-sphere asymptotically.

The formula

$$
\frac{d}{d \lambda} H_{X}=\int_{\infty}\left[\frac{d}{d \lambda} Q_{X}(g)-i_{X} \theta\left(g ; \frac{d}{d \lambda} g\right)\right],
$$

can be shown to define [39] a conserved quantity $H_{X}$. If $X^{a} \rightarrow(\partial / \partial t)^{a}$, then $H_{X}$ is equal to the ADM-mass $M$; if $X^{a} \rightarrow\left(\partial / \partial x^{i}\right)^{a}$ (asymptotic translation), then $-H_{X}$ is equa 15 to the linear ADM-momentum $P_{i}$; if $X^{a} \rightarrow x^{i}\left(\partial / \partial x^{j}\right)^{a}-x^{j}\left(\partial / \partial x^{i}\right)^{a}$ (asymptotic rotation), then $-H_{X}$ is equal to the ADM-angular momentum $J_{[i j]}$ in the $i j$-plane 16 ; if $X^{a} \rightarrow t\left(\partial / \partial x^{i}\right)^{a}+x^{i}(\partial / \partial t)^{a}$ (asymptotic boost), then $H_{X}$ is equal to the ADM-center of mass, $C_{i}$. Together, the $\frac{1}{2} D(D+1)$ quantities $(M, \mathbf{P}, \mathbf{J}, \mathbf{C})$ will be referred to as "ADM conserved quantities" associated with the spacetime $\left(\mathscr{M}, g_{a b}\right)$.

Now consider the case $X^{a}=K^{a}$, where $K^{a}$ is the horizon Killing field of $g_{a b}(0)$. Then $K^{a}$ takes the coordinate form (13) at the horizon for all $\lambda$. In particular, $\left.K^{a}\right|_{B}=0$ and, thus, $i_{K} \theta=0$ on $B$. On the other hand, using (5), we obtain

$$
\int_{B} Q_{K}(g(\lambda))=\frac{\kappa}{8 \pi} A(\lambda)
$$

where $A(\lambda)$ is the area of $B$ in the metric $g_{a b}(\lambda)$. Thus, we obtain

$$
\int_{B}\left[\frac{d}{d \lambda} Q_{K}(g)-i_{K} \theta\left(g ; \frac{d}{d \lambda} g\right)\right]=\frac{\kappa}{8 \pi} \frac{d A}{d \lambda},
$$

which holds for the metric $g_{a b} \equiv g_{a b}(\lambda)$. By eqs. (12) and (37), we have

$$
\int_{\infty}\left[\frac{d}{d \lambda} Q_{K}(g)-i_{K} \theta\left(g ; \frac{d}{d \lambda} g\right)\right]=\frac{d}{d \lambda} M-\sum_{A} \Omega_{A} \frac{d}{d \lambda} J_{A} .
$$

\footnotetext{
${ }^{15}$ The map $X \rightarrow H_{X}$ is a linear functional and thus is a "covector". To get the $D$-dimensional energymomentum vector, we have to "raise the index" with the Minkowskian metric. This accounts for the relative minus sign between $M$ as compared with $P_{i}$ and $J_{[i j]}$ in their definitions in terms of $H_{X}$.

${ }^{16}$ The notation $J_{A}$ used above refers to $N$ distinguished mutually orthogonal 2-planes defined by the $N$ rotational Killing fields $\psi_{A}^{a}$.
} 
Combining eqs.(36), (39), and (40), we obtain

$$
W_{\Sigma}\left(g ; \frac{d}{d \lambda} g, \mathscr{L}_{K} g\right)=\frac{d}{d \lambda} M-\sum_{A} \Omega_{A} \frac{d}{d \lambda} J_{A}-\frac{\kappa}{8 \pi} \frac{d}{d \lambda} A .
$$

This equation holds for all $\lambda$, with $\kappa$ and $\Omega_{A}$ fixed constants, equal, respectively, to the surface gravity and angular velocities of the stationary black hole metric $g_{a b}(0)$. Since $K^{a}$ is a Killing field of $g_{a b}(0)$, when $\lambda=0$ we have $\mathscr{L}_{K} g=0$ and eq. (41) reduces to the first law of black hole mechanics

$$
0=\delta M-\sum_{A} \Omega_{A} \delta J_{A}-\frac{\kappa}{8 \pi} \delta A
$$

where we remind the reader that our use of the notation " $\delta$ " here (rather than " $d / d \lambda$ ") indicates that this equation holds only at $\lambda=0$.

Finally, we consider the gauge dependence of $W_{\Sigma}$. It is clear that $W_{\Sigma}\left(g ; \delta_{1} g, \delta_{2} g\right)$ will be gauge invariant if and only if $W_{\Sigma}\left(g ; \delta g, \mathscr{L}_{\xi} g\right)=0$ for all allowed $\delta g_{a b}$ and $\xi^{a}$. It follows immediately from integrating (34) over $\Sigma$ (with $E=0$ ) that if $\delta g_{a b}$ satisfies the linearized constraints, then $W_{\Sigma}\left(g ; \delta g, \mathscr{L}_{\xi} g\right)=0$ for all $\xi^{a}$ of compact support 17 away from $B$. Thus, for solutions, $W_{\Sigma}\left(g ; \delta_{1} g, \delta_{2} g\right)$ is gauge invariant for gauge transformations of compact support. However, if $\xi^{a}$ is a smooth vector field such that $\mathscr{L}_{\xi} g_{a b}$ satisfies our asymptotic conditions at infinity (i.e., $\xi^{a}$ is an asymptotic symmetry at infinity) and our boundary conditions at $B$, then $W_{\Sigma}\left(g ; \delta g, \mathscr{L}_{\xi} g\right)$ need not vanish for all $\delta g$ that satisfy our asymptotic conditions at infinity and our boundary conditions at $B$. The following lemma characterizes the extent to which $W_{\Sigma}$ is gauge invariant. This lemma will play a critical role in our analysis.

Lemma 2. Let $\delta g_{a b}$ be a solution to the linearized Einstein equations around our stationary black hole background $g_{a b}$ satisfying our asymptotic flatness conditions and our gauge conditions (7) and (11) at $B$. Suppose in addition that $\delta A=0$ (so that, by (11), we have $\left.\delta \epsilon\right|_{B}=0$ ) and that $\delta H_{X}=0$ for some asymptotic symmetry $X^{a}$. Then $W_{\Sigma}\left(g ; \delta g, \mathscr{L}_{\xi} g\right)=0$ for all smooth $\xi^{a}$ such that (i) $\left.\xi^{a}\right|_{B}$ is tangent to the generators of $\mathscr{H}$ and (ii) $\xi^{a}$ approaches a multiple of $X^{a}$ as $\rho \rightarrow \infty$. Conversely, if $\delta g_{a b}$ is smooth and asymptotically flat and if $W_{\Sigma}\left(g ; \delta g, \mathscr{L}_{\xi} g\right)=0$ for all such $\xi^{a}$, then $\delta g_{a b}$ is a solution to the linearized Einstein equation with $\left.\delta \vartheta\right|_{B}=\left.\delta \epsilon\right|_{B}=0$ at $B$ and with $\delta H_{X}=0$.

A proof of this lemma is given in appendix A.

\subsection{Second variations and canonical energy}

Returning to eq. (41), we write out the $\lambda$-dependence explicitly as

$$
W_{\Sigma}\left(g(\lambda) ; \frac{d}{d \lambda} g(\lambda), \mathscr{L}_{K} g(\lambda)\right)=\frac{d}{d \lambda} M(\lambda)-\sum_{A} \Omega_{A} \frac{d}{d \lambda} J_{A}(\lambda)-\frac{\kappa}{8 \pi} \frac{d}{d \lambda} A(\lambda),
$$

\footnotetext{
${ }^{17}$ Conversely, if $W_{\Sigma}\left(g ; \delta g, \mathscr{L}_{\xi} g\right)=0$ for all $\xi^{a}$ of compact support away from $B$, then $\delta g_{a b}$ satisfies the linearized constraints. These facts, which are manifestations of the familiar statement that in general relativity "the constraints generate gauge transformations," will be exploited heavily in subsection 4.1 below.
} 
where it should be emphasized that $\kappa$ and $\Omega_{A}$ do not depend on $\lambda$. We now take a $\lambda$ derivative of this equation, and then set $\lambda=0$. Using the fact that $\mathscr{L}_{K} g_{a b}(0)=0$, we get

$$
W_{\Sigma}\left(g ; \gamma, \mathscr{L}_{K} \gamma\right)=\left.\frac{d^{2}}{d \lambda^{2}} M(\lambda)\right|_{\lambda=0}-\left.\sum_{A} \Omega_{A} \frac{d^{2}}{d \lambda^{2}} J_{A}(\lambda)\right|_{\lambda=0}-\left.\frac{\kappa}{8 \pi} \frac{d^{2}}{d \lambda^{2}} A(\lambda)\right|_{\lambda=0},
$$

where $\gamma_{a b}=d g_{a b} /\left.d \lambda\right|_{\lambda=0}=\delta g_{a b}(0)$ is the first order perturbation of the black hole spacetime. Writing $K^{a}=t^{a}+\sum_{A} \Omega_{A} \psi_{A}^{a}$, the left side may be written as

$$
W_{\Sigma}\left(g ; \gamma, \mathscr{L}_{K} \gamma\right)=\mathcal{E}+\sum_{A} \Omega_{A} W_{\Sigma}\left(g ; \gamma, \mathscr{L}_{\psi_{A}} \gamma\right)
$$

where we have defined the canonical energy by

$$
\mathcal{E} \equiv W_{\Sigma}\left(g ; \gamma, \mathscr{L}_{t} \gamma\right)
$$

Since $t^{a}$ is a Killing field of the background, $\mathscr{L}_{t}$ commutes with the linearized Einstein operator, so $\mathscr{L}_{t} \gamma_{a b}$ satisfies the linearized field equations whenever $\gamma_{a b}$ does. $\mathscr{L}_{t} \gamma_{a b}$ also satisfies our asymptotic conditions at infinity (with faster fall-off) and our boundary conditions at $B$. Hence, it follows from the conservation of symplectic product that $\mathcal{E}$ is conserved for all solutions $\gamma_{a b}$ in the sense that it takes the same value if evaluated on another Cauchy surface $\Sigma^{\prime}$ extending from $B$ to spatial infinity. We may rewrite (44) as

$$
\mathcal{E}=-\sum_{A} \Omega_{A} W_{\Sigma}\left(g ; \gamma, \mathscr{L}_{\psi_{A}} \gamma\right)+\delta^{2} M-\sum_{A} \Omega_{A} \delta^{2} J_{A}-\frac{\kappa}{8 \pi} \delta^{2} A
$$

where we have written $\delta^{2} M=d^{2} M /\left.d \lambda^{2}\right|_{\lambda=0}$, etc. Note that $\mathcal{E}$ and $W_{\Sigma}\left(g ; \gamma, \mathscr{L}_{\psi_{A}} \gamma\right)$ depend only upon $\gamma_{a b}=\delta g_{a b}$, whereas $\delta^{2} M, \delta^{2} J_{A}$, and $\delta^{2} A$ depend upon the second order perturbation $\delta^{2} g_{a b}=d^{2} g_{a b} /\left.d \lambda^{2}\right|_{\lambda=0}$. However, the difference between two second order perturbations will be a homogeneous solution of the first order perturbation equations, so its net contribution to (47) will vanish by the first law of black hole mechanics (42).

We summarize our main result thus far as follows:

Proposition 1. (Second variation formula): Let $\left(\mathscr{M}, g_{a b}(\lambda)\right)$ be a one-parameter family of smooth, asymptotically flat vacuum solutions such that $g_{a b}(0)$ is a stationary black hole spacetime with bifurcate Killing horizon. Suppose that the gauge condition (5) has been imposed near the horizon $\mathscr{H}^{+}$. Then the canonical energy (46) of $\gamma_{a b}$ satisfies (47), where $\Omega_{A}$ and $\kappa$ are the angular velocities and surface gravity of the horizon of the background solution $g_{a b}(0)$. by

It is useful to view the canonical energy as a bilinear form on perturbations, defined

$$
\mathcal{E}\left(\gamma_{1}, \gamma_{2}\right) \equiv W_{\Sigma}\left(g ; \gamma_{1}, \mathscr{L}_{t} \gamma_{2}\right)
$$

so that the canonical energy originally defined by (46) is given by $\mathcal{E}=\mathcal{E}(\gamma, \gamma)$. Although it is not manifest from its definition, it is easily seen that $\mathcal{E}\left(\gamma_{1}, \gamma_{2}\right)$ is symmetric in $\left(\gamma_{1}, \gamma_{2}\right)$ :

Proposition 2. Let $\gamma_{1}$ and $\gamma_{2}$ be smooth, asymptotically flat solutions to the linearized field equations on the stationary black hole background $g_{a b}(0)$. Then, we have

$$
\mathcal{E}\left(\gamma_{1}, \gamma_{2}\right)=\mathcal{E}\left(\gamma_{2}, \gamma_{1}\right)
$$


Proof: Since the background metric $g$ is stationary, it follows immediately from the antisymmetry of the symplectic current $\omega$ that

$$
\omega\left(g ; \gamma_{1}, \mathscr{L}_{t} \gamma_{2}\right)-\omega\left(g ; \gamma_{2}, \mathscr{L}_{t} \gamma_{1}\right)=\mathscr{L}_{t} \omega\left(g ; \gamma_{1}, \gamma_{2}\right)
$$

Now we use the standard identity $\mathscr{L}_{X}=i_{X} \mathrm{~d}+\mathrm{d} i_{X}$ for the Lie-derivative acting on forms, together with the fact that $\mathrm{d} \omega=0$ when $\gamma_{1}$ and $\gamma_{2}$ are linearized solutions. Integrating over $\Sigma$, we obtain

$$
\mathcal{E}\left(\gamma_{1}, \gamma_{2}\right)-\mathcal{E}\left(\gamma_{2}, \gamma_{1}\right)=\int_{\infty} i_{t} \omega\left(g ; \gamma_{1}, \gamma_{2}\right)-\int_{B} i_{t} \omega\left(g ; \gamma_{1}, \gamma_{2}\right)
$$

However, the contribution from infinity vanishes on account of our asymptotic conditions, whereas the contribution from $B$ vanishes because $t^{a}$ is tangent to $B$. Thus, $\mathcal{E}$ is symmetric.

In our analysis of the next sections, we will be concerned with the positivity properties of $\mathcal{E}$ as well as fluxes of $\mathcal{E}$ through the horizon and at null infinity. Unfortunately, for a rotating black hole, the term $\sum_{A} \Omega_{A} W_{\Sigma}\left(g ; \gamma, \mathscr{L}_{\psi_{A}} \gamma\right)$ will spoil any positivity properties of $\mathcal{E}$. Furthermore, the fact that, for the case of a rotating black hole, $t^{a}$ is spacelike at the horizon will preclude the possibility of having a positive flux of canonical energy through the horizon 18 . Fortunately, both difficulties can be resolved by restricting consideration to axisymmetric perturbations

$$
\mathscr{L}_{\psi_{A}} \gamma=0
$$

and we shall impose this restriction later in our analysis. For axisymmetric perturbations, (47) reduces to

$$
\mathcal{E}=\delta^{2} M-\sum_{A} \Omega_{A} \delta^{2} J_{A}-\frac{\kappa}{8 \pi} \delta^{2} A
$$

An important property of axisymmetric perturbations is given by the following proposition:

Proposition 3. The canonical energy is gauge invariant with respect to gauge transformations that preserve the gauge conditions of subsection 2.1 when restricted to the space of axisymmetric perturbations that satisfy the linearized field equations and have vanishing linearized $\mathrm{ADM}$ linear momenta, $\delta P_{i}=0$, and vanishing change of area, $\delta A=0$.

Proof: The gauge transformations that preserve the gauge conditions of subsection 2.1 and axisymmetry are $\gamma_{a b} \rightarrow \gamma_{a b}+\mathscr{L}_{X} g_{a b}$ where $X^{a}$ satisfies the conditions in the remark below lemma 1 of subsection 2.1 and, in addition, satisfies $\mathscr{L}_{\psi_{A}} X^{a}=0$ everywhere. Since, by Proposition $2, \mathcal{E}\left(\gamma_{1}, \gamma_{2}\right)$ is symmetric in $\left(\gamma_{1}, \gamma_{2}\right)$, gauge invariance will be proven if we can show that

$$
\mathcal{E}\left(\gamma, \mathscr{L}_{X} g\right)=W_{\Sigma}\left(\gamma, \mathscr{L}_{t} \mathscr{L}_{X} g\right)=0
$$

\footnotetext{
${ }^{18}$ We could have defined a canonical energy $\mathcal{E}_{K}$ with respect to the horizon Killing field $K^{a}$ instead of $t^{a}$, in which case the term $\sum_{A} \Omega_{A} W_{\Sigma}\left(g ; \gamma, \mathscr{L}_{\psi_{A}} \gamma\right)$ would not have appeared in the expression for $\mathcal{E}_{K}$ and the flux through the horizon would be positive. However, in the absence of axisymmetry, the spacelike character of $K^{a}$ near infinity for a rotating black hole will similarly spoil the positivity properties of $\mathcal{E}_{K}$ and preclude the possibility of its having a positive flux through null infinity
} 
for all solutions $\gamma_{a b}$ for which $\delta P_{i}=0$ and $\delta A=0$. However, we have

$$
\mathscr{L}_{t} \mathscr{L}_{X} g_{a b}=\left(\mathscr{L}_{t} \mathscr{L}_{X}-\mathscr{L}_{X} \mathscr{L}_{t}\right) g_{a b}=\mathscr{L}_{[t, X]} g_{a b},
$$

since $\mathscr{L}_{t} g_{a b}=0$. Since $X^{a}$ is an asymptotic symmetry, $[t, X]^{a}$ is an asymptotic spatial translation. Furthermore, since $t^{a}=K^{a}-\sum \Omega_{A} \psi^{a}{ }_{A}$ and $\left[\psi_{A}, X\right]^{a}=\mathscr{L}_{\psi_{A}} X^{a}=0$, it follows that $\left.[t, X]^{a}\right|_{B}$ is normal to $\mathscr{H}^{+}$at $B$. The proposition now follows immediately from lemma 2 applied to $\xi^{a}=[t, X]^{a}$.

In our later arguments below we will need to consider perturbations towards other stationary black holes. We now explain the subtleties involved in this notion and give a precise definition of it. It might appear to be obvious from its definition (46) that $\mathcal{E}$ must vanish for stationary perturbations. However, this is not the case because, as already indicated at the end of subsection 2.1, we cannot assume that the stationary Killing field $t^{a}(\lambda)$ of the varied spacetime coincides with $t^{a}$. Indeed, the example of the one-parameter family of Schwarzschild metrics of mass $M=M_{0}+\alpha \lambda$ in 4-dimensions illustrates that the canonical energy need not vanish for perturbations to other stationary black holes. For this family, we have $\delta^{2} M=0$, but $A(\lambda)=16 \pi\left(M_{0}+\alpha \lambda\right)^{2}$, so $\delta^{2} A=32 \pi \alpha^{2}$. Thus, by (53), we have (using $\kappa=1 / 4 M_{0}$ )

$$
\mathcal{E}=-\alpha^{2} / M_{0}<0
$$

To see why this result is compatible with (46) and to find the appropriate conditions to express the notion that a perturbation $\gamma_{a b}$ represents a perturbation towards another stationary black hole, let $g_{a b}(\lambda)$ be a one-parameter family of stationary black holes, with stationary Killing field $t^{a}(\lambda)$, horizon Killing field $K^{a}(\lambda)$, and axial Killing fields $\psi_{A}{ }^{a}(\lambda)$ associated with a nonvanishing angular velocity of the horizon. As already stated at the end of subsection 2.1, it is compatible with our other gauge conditions to assume that the axial Killing fields $\psi_{A}{ }^{a}(\lambda)$ are independent of $\lambda$, so that $\psi_{A}{ }^{a}(\lambda)=\psi_{A}{ }^{a}$. We may also assume that near infinity, we have $t^{a}(\lambda)=t^{a}$. However, since $K^{a}$ takes the form (13) on $\mathscr{H}^{+}$, we must have $K^{a}(\lambda) \neq K^{a}$ near $\mathscr{H}^{+}$if the surface gravity, $\kappa(\lambda)$, of $g_{a b}(\lambda)$ differs from $\kappa$. Nevertheless, we may assume that $\kappa K^{a}(\lambda)=\kappa(\lambda) K^{a}$ near $\mathscr{H}^{+}$. If we make this choice, then, near $\mathscr{H}^{+}$we have

$$
t^{a}(\lambda)=\frac{\kappa(\lambda)}{\kappa} K^{a}-\sum_{A} \Omega_{A}(\lambda) \psi_{A}^{a},
$$

so $t^{a}(\lambda)$ must differ from $t^{a}$ near $\mathscr{H}^{+}$if there is any change in the surface gravity or angular velocities of the horizon.

Thus, if $g_{a b}(\lambda)$ is a one parameter family of stationary black holes, then we can choose a gauge compatible with our previous choices such that $\psi_{A}{ }^{a}(\lambda)=\psi_{A}{ }^{a}, t^{a}(\lambda)=t^{a}$ near infinity, and $t^{a}(\lambda)$ is given by (57) near $\mathscr{H}^{+}$. The perturbation $\gamma_{a b}=d g_{a b} /\left.d \lambda\right|_{\lambda=0}$ must therefore satisfy $\mathscr{L}_{\psi_{A}} \gamma_{a b}=0$ for all $\psi_{A}{ }^{a}$ appearing in (57) and

$$
\mathscr{L}_{t} \gamma_{a b}+\mathscr{L}_{\delta t} g_{a b}=0
$$

where, near infinity, we have $\delta t^{a}=0$ whereas near $\mathscr{H}^{+}$, we have

$$
\delta t^{a}=c t^{a}+\sum_{A} b_{A} \psi_{A}^{a}
$$


where $c$ and $b_{A}$ are constants. Thus, near $\mathscr{H}^{+}, \delta t^{a}$ is of the form of a Killing field of the background metrie19.

Thus, rather than having to vanish, we see that $\mathscr{L}_{t} \gamma_{a b}$ must take the form $\mathscr{L}_{Y} g_{a b}$ where $Y^{a}$ vanishes near infinity and is a linear combination of Killing fields (59) near $\mathscr{H}^{+}$. Equivalently, writing $Z^{a}=Y^{a}-c t^{a}-\sum_{A} b_{A} \psi_{A}{ }^{a}$ and noting that $\mathscr{L}_{Y} g_{a b}=\mathscr{L}_{Z} g_{a b}$, we see that a perturbation towards a stationary black hole can be put in a gauge compatible with our gauge conditions such that

$$
\mathscr{L}_{t} \gamma_{a b}=\mathscr{L}_{Z} g_{a b}
$$

where $Z^{a}$ vanishes near $\mathscr{H}^{+}$and near infinity takes the form

$$
Z^{a}=c t^{a}+\sum_{A} b_{A} \psi_{A}^{a}
$$

and where, furthermore, $\mathscr{L}_{\psi_{A}} \gamma_{a b}=0$ for all $\psi_{A}{ }^{a}$ appearing in (12) and/or (61). In a general gauge satisfying our gauge conditions, we have

$$
\mathscr{L}_{t} \gamma_{a b}=\mathscr{L}_{\xi} g_{a b}+\mathscr{L}_{t} \mathscr{L}_{X} g_{a b}
$$

where $X^{a}$ satisfies the conditions of the remark below lemma 1. Thus, taking account of (55) and the remarks below that equation, we have have motivated the following definition:

Definition 2.1. A smooth, asymptotically flat, axisymmetric solution $\gamma_{a b}$ of the linearized field equations is said to be a perturbation towards a stationary black hole if

$$
\mathscr{L}_{t} \gamma_{a b}=\mathscr{L}_{\xi} g_{a b}
$$

where $\left.\xi^{a}\right|_{B}$ is normal to $\mathscr{H}^{+}$and near infinity takes the form

$$
\xi^{a}=c t^{a}+\sum_{A} b_{A} \psi_{A}^{a}+\sum_{i} a_{i}\left(\frac{\partial}{\partial x^{i}}\right)^{a} .
$$

Note that although the linearization of any one-parameter family of stationary black holes must satisfy definition 2.1, our definition does not require that there actually exist a one-parameter family $g_{a b}(\lambda)$ of stationary black holes corresponding to $\gamma_{a b}$. In any case, the key point about perturbations towards a stationary black hole is that they are stationary and thus are benign with regard to linear stability of the background black hole. An important property of perturbations towards a stationary black holes is the following:

Proposition 4. Let $\gamma_{1}$ be a perturbation for which $\delta M=\delta J_{A}=\delta P_{i}=0$ (and hence, by the first law of black hole mechanics $\delta A=0$ ), and let $\gamma_{2}$ be a perturbation towards a stationary black hole. Then $\mathcal{E}\left(\gamma_{1}, \gamma_{2}\right)=0$.

\footnotetext{
${ }^{19}$ The sum on the right side of (59) is allowed to include rotational Killing fields $\psi_{A}{ }^{a}$ of the background that may not have appeared in (12) because $\Omega_{A}(\lambda=0)=0$.
} 
Proof: We have

$$
\mathcal{E}\left(\gamma_{1}, \gamma_{2}\right)=W_{\Sigma}\left(g ; \gamma_{1}, \mathscr{L}_{t} \gamma_{2}\right)=W_{\Sigma}\left(g ; \gamma_{1}, \mathscr{L}_{\xi} g\right)=0
$$

where the second equality merely substitutes the definition of a perturbations towards a stationary black hole and the last equality is an immediate consequence of lemma 2 .

In subsection 4.1 below, we will considerably strengthen this result by showing that, when considered as a quadratic form on a suitable space of smooth, axisymmetric solutions to the linearized field equations for which $\delta M=\delta J_{A}=\delta P_{i}=0$, the canonical energy $\mathcal{E}$ will be degenerate precisely on the perturbations towards stationary black holes. This will play a key role in our stability arguments.

\subsection{Evaluation of canonical energy}

In the previous section, we obtained a simple formula for $\mathcal{E}$ in terms of second order variations of mass, angular momentum, and area. However, in order to use this formula to evaluate $\mathcal{E}$, we must calculate second order perturbation $d^{2} g_{a b} /\left.d \lambda^{2}\right|_{\lambda=0}$, even though $\mathcal{E}$ really only depends on the first order perturbation $\gamma_{a b}=d g_{a b} /\left.d \lambda\right|_{\lambda=0}$. It is useful to have a formula for $\mathcal{E}$ that expresses it directly in terms of $\gamma_{a b}$, and/or the initial data, $\left(\delta h_{a b}, \delta p^{a b}\right)$, for $\gamma_{a b}$. Such a formula can be obtained from the original definition (48),

$$
\mathcal{E}\left(\gamma_{1}, \gamma_{2}\right)=W_{\Sigma}\left(g ; \gamma_{1}, \mathscr{L}_{t} \gamma_{2}\right)=-\frac{1}{16 \pi} \int_{\Sigma}\left(\delta_{1} h_{a b} \mathscr{L}_{t} \delta_{2} p^{a b}-\delta_{1} p^{a b} \mathscr{L}_{t} \delta_{2} h_{a b}\right)
$$

by substituting the explicit expressions (21) and (22). However, to put the right side in a more useful form, we will follow a different strategy and return to the fundamental identity (34), using the fact that the variation is being taken about a solution, so $E=0$. We obtain at $\lambda=0$

$$
\omega\left(g ; \delta g, \mathscr{L}_{X} g\right)=\delta C_{X}(g)+\mathrm{d}\left[\delta Q_{X}-i_{X} \theta(g ; \delta g)\right] .
$$

The constraints $C_{X}=X^{a} C_{a}$ are given by eq. (32) above. In terms of the variables $\left(h_{a b}, p^{a b}\right)$, the constraints take the form 20

$$
C_{a}=\left(\begin{array}{c}
C_{a} \nu^{a} \\
C_{b} h_{a}{ }^{b}
\end{array}\right):=\frac{1}{16 \pi} h^{\frac{1}{2}}\left(\begin{array}{c}
-R(h)+h^{-1} p_{a b} p^{a b}-\frac{1}{D-2} h^{-1} p^{2} \\
-2 D_{b}\left(h^{-\frac{1}{2}} p_{a}^{b}\right)
\end{array}\right)=0,
$$

where $\nu^{a}$ denotes the future-directed unit normal to $\Sigma$. The first line of this equation corresponds to the Hamiltonian constraint and the second line corresponds to the momentum constraint. The linearized constraints $\delta C_{a}$ may be viewed as the result of acting on $\left(\delta h_{a b}, \delta p^{a b}\right)$ by a linear operator, $\mathcal{L}$,

$$
\delta C_{a}=\mathcal{L}\left(\begin{array}{l}
\delta h_{a b} \\
\delta p^{a b}
\end{array}\right)
$$

\footnotetext{
${ }^{20}$ Here and below, we omit writing the factor of the non-dynamical coordinate $(D-1)$-form $e_{0}$ on $\Sigma$ in the expression for $C_{a}$ [see the discussion below (27)].
} 
where $\mathcal{L}$ is explicitly given by

$$
16 \pi \mathcal{L}\left(\begin{array}{l}
\delta h_{a b} \\
\delta p^{a b}
\end{array}\right)=\left(\begin{array}{c}
h^{\frac{1}{2}}\left(D^{a} D_{a} \delta h_{c}{ }^{c}-D^{a} D^{b} \delta h_{a b}+R^{a b}(h) \delta h_{a b}\right)+ \\
h^{-\frac{1}{2}}\left(-\delta h_{c}{ }^{c} p^{a b} p_{a b}+2 p_{a b} \delta p^{a b}+2 p^{a c} p^{b} \delta h_{b c}+\right. \\
\left.\frac{1}{D-2} p^{c}{ }_{c} p^{d}{ }_{d} \delta h^{a}{ }_{a}-\frac{2}{D-2} p^{a}{ }_{a} \delta p^{b}{ }_{b}-\frac{2}{D-2} \delta h_{a b} p^{a b} p_{c}{ }^{c}\right) \\
-2 h^{\frac{1}{2}} D^{b}\left(h^{-\frac{1}{2}} \delta p_{a b}\right)+D_{a} \delta h_{c b} p^{c b}-2 D_{c} \delta h_{a b} p^{b c}
\end{array}\right) .
$$

Since $\mathcal{L}$ is a differential operator that maps the pair $\left(\delta h_{a b}, \delta p^{a b}\right)$ consisting of a symmetric tensor, $\delta h_{a b}$, and a symmetric tensor density, $\delta p^{a b}$, on $\Sigma$ into a pair $\left(\tilde{N}, \tilde{N}_{a}\right)$ consisting of a scalar density and dual vector density on $\Sigma$, its adjoint differential operator, $\mathcal{L}^{*}$, maps a pair $\left(N, N^{a}\right)$ consisting of a scalar and vector field on $\Sigma$ into a pair $\left(\delta \tilde{h}^{a b}, \delta \tilde{p}_{a b}\right)$ consisting of a symmetric tensor density and symmetric tensor on $\Sigma$. In other words, $\mathcal{L}, \mathcal{L}^{*}$ are maps

$$
\begin{aligned}
& \mathcal{L}: C^{\infty}\left(\Sigma,\left(T^{*} \Sigma\right)^{\vee 2}\right) \oplus C^{\infty}\left(\Sigma,(T \Sigma)^{\vee 2} \otimes \Lambda^{\frac{1}{2}}\right) \rightarrow C^{\infty}\left(\Sigma, \Lambda^{\frac{1}{2}}\right) \oplus C^{\infty}\left(\Sigma, T^{*} \Sigma \otimes \Lambda^{\frac{1}{2}}\right) \\
& \mathcal{L}^{*}: C^{\infty}(\Sigma, \mathbb{R}) \oplus C^{\infty}(\Sigma, T \Sigma) \rightarrow C^{\infty}\left(\Sigma,(T \Sigma)^{\vee 2} \otimes \Lambda^{\frac{1}{2}}\right) \oplus C^{\infty}\left(\Sigma,\left(T^{*} \Sigma\right)^{\vee 2}\right)
\end{aligned}
$$

where $\Lambda^{\frac{1}{2}}$ is the line bundle of densities of weight $\frac{1}{2}$, and $\vee$ is the symmetric tensor product. $\mathcal{L}^{*}$ is uniquely determined as a differential operator by the requirement that for all smooth $\left(\delta h_{a b}, \delta p^{a b}\right)$ of compact support on $\Sigma \backslash B$ and all smooth $\left(N, N^{a}\right)$, we have

$$
\left\langle\mathcal{L}^{*}\left(\begin{array}{c}
N \\
N^{a}
\end{array}\right) \mid\left(\begin{array}{c}
\delta h_{a b} \\
\delta p^{a b}
\end{array}\right)\right\rangle=\left\langle\left(\begin{array}{c}
N \\
N^{a}
\end{array}\right) \mid \mathcal{L}\left(\begin{array}{l}
\delta h_{a b} \\
\delta p^{a b}
\end{array}\right)\right\rangle .
$$

Here, the angle brackets denote the natural $L^{2}$-type inner product on the appropriate tensor/tensor density pairs, e.g.

$$
\left\langle\left(\begin{array}{c}
N \\
N^{a}
\end{array}\right) \mid\left(\begin{array}{c}
\tilde{N} \\
\tilde{N}_{a}
\end{array}\right)\right\rangle=\int_{\Sigma}\left(N \tilde{N}+N^{a} \tilde{N}_{a}\right) .
$$

One can straightforwardly calculate that $\mathcal{L}^{*}$ is given by

$$
16 \pi \mathcal{L}^{*}\left(\begin{array}{c}
N \\
N^{a}
\end{array}\right)=\left(\begin{array}{c}
h^{\frac{1}{2}}\left(-D^{c} D_{c} N h^{a b}+D^{a} D^{b} N+R^{a b}(h) N\right)+ \\
h^{-\frac{1}{2}}\left(-h^{a b} p^{c d} p_{c d} N+2 p^{(a}{ }_{c} p^{b)} N+\frac{1}{D-2} h^{a b} p_{c}^{c} p^{d}{ }_{d} N\right. \\
\left.-\frac{2}{D-2} p^{a b} p^{c}{ }_{c} N-p^{a b} D_{c} N^{c}+2 D_{c} N^{(a} p^{b) c}\right) \\
h^{-\frac{1}{2}}\left(2 p_{a b} N-\frac{2}{D-2} h_{a b} p^{c}{ }_{c} N\right)+2 D_{(a} N_{b)}
\end{array}\right)
$$

Comparing (72) with the integral of (67) over $\Sigma$ with $(\delta h, \delta p)$ of compact support on $\Sigma \backslash B$, we see that

$$
W_{\Sigma}\left(g ; \delta g, \mathscr{L}_{X} g\right)=\left\langle\mathcal{L}^{*}(X) \mid\left(\begin{array}{l}
\delta h \\
\delta p
\end{array}\right)\right\rangle
$$

Here by $\mathcal{L}^{*}(X)$, we mean $\mathcal{L}^{*}\left(N, N^{a}\right)$, where

$$
X^{a}=N \nu^{a}+N^{a}
$$


is the usual decomposition of a vector into its lapse and shift. Since neither side of (75) contains derivatives of $(\delta h, \delta p)$, we can solve for $\mathscr{L}_{X} g$, obtaining

$$
\mathscr{L}_{X}\left(\begin{array}{l}
h \\
p
\end{array}\right)=16 \pi \sigma \mathcal{L}^{*}(X), \quad \sigma \equiv\left(\begin{array}{cc}
0 & h_{a c} h_{b d} \\
-h^{a c} h^{b d} & 0
\end{array}\right)
$$

which corresponds to the usual ADM evolution equations. Note that - in view of (77) (75) continues to hold even when $(\delta h, \delta p)$ is not of compact support on $\Sigma \backslash B$, provided only that the integrals defining the left and right sides converge. Note also that when $(\delta h, \delta p)$ is not of compact support on $\Sigma \backslash B$, integration of (67) over $\Sigma$ yields at $\lambda=0$

$$
\begin{aligned}
\left\langle\mathcal{L}^{*}(X) \mid\left(\begin{array}{l}
\delta h \\
\delta p
\end{array}\right)\right\rangle= & \left\langle X \mid \mathcal{L}\left(\begin{array}{l}
\delta h \\
\delta p
\end{array}\right)\right\rangle+\int_{\infty}\left[\delta Q_{X}(g)-i_{X} \theta(g ; \delta g)\right] \\
& -\int_{B}\left[\delta Q_{X}(g)-i_{X} \theta(g ; \delta g)\right]
\end{aligned}
$$

To derive the desired formula for $\mathcal{E}\left(\gamma_{1}, \gamma_{2}\right)$, we now consider a two-parameter family of metrics $g_{a b}\left(\lambda_{1}, \lambda_{2}\right)=g_{a b}+\lambda_{1} \gamma_{1 a b}+\lambda_{2} \gamma_{2 a b}$, where $g_{a b}$ is the background metric and $\gamma_{1 a b}$ and $\gamma_{2 a b}$ solve the linearized equations. Although $g_{a b}\left(\lambda_{1}, \lambda_{2}\right)$ is not a solution, it does satisfy the field equations to first order in both $\lambda_{1}$ and $\lambda_{2}$. We find that the corrections to (75) away from $\lambda_{1}=\lambda_{2}=0$ are of the form

$$
W_{\Sigma}\left(g\left(\lambda_{1}, \lambda_{2}\right) ; \frac{\partial}{\partial \lambda_{1}} g\left(0, \lambda_{2}\right), \mathscr{L}_{X} g\left(\lambda_{1}, \lambda_{2}\right)\right)=\left\langle\mathcal{L}^{*}(X) \mid\left(\begin{array}{c}
\frac{\partial}{\partial \lambda_{1}} h \\
\frac{\partial}{\partial \lambda_{1}} p
\end{array}\right)\right\rangle+O\left(\lambda_{1}\right)+O\left(\lambda_{2}^{2}\right) .
$$

Taking the derivative of this equation with respect to $\lambda_{2}$, setting $\lambda_{1}=\lambda_{2}=0$, and then also setting $X^{a}=t^{a}$, we obtain

$$
\mathcal{E}\left(\gamma_{1}, \gamma_{2}\right)=W_{\Sigma}\left(g ; \gamma_{1}, \mathscr{L}_{t} \gamma_{2}\right)=\left.\left\langle\frac{\partial}{\partial \lambda_{2}} \mathcal{L}^{*}(t) \mid\left(\begin{array}{c}
\frac{\partial}{\partial \lambda_{1}} h \\
\frac{\partial}{\partial \lambda_{1}} p
\end{array}\right)\right\rangle\right|_{\lambda_{1}=\lambda_{2}=0} .
$$

The right side of this equation can be computed by taking the variation of the right side of (74), although we shall not explicitly write out the resulting formula here. Note that although $\delta t^{a}=0$, it is not compatible with the gauge conditions we have already imposed near the horizon to require $\delta \nu^{a}=0$, so variations of the lapse and shift must be taken into account in the calculation of $\left.\frac{\partial}{\partial \lambda_{2}} \mathcal{L}^{*}(t)\right|_{\lambda_{1}=\lambda_{2}=0}$.

An alternative formula for $\mathcal{E}$ can be derived by noting that, to the desired accuracy, one may substitute (78) on the right side of (79). Taking the derivative of the resulting equation with respect to $\lambda_{2}$, and again setting $\lambda_{1}=\lambda_{2}=0$ as well as $X=t$, we obtain

$$
\begin{aligned}
\mathcal{E}\left(\gamma_{1}, \gamma_{2}\right) & =\left.\left\langle t \mid \frac{\partial}{\partial \lambda_{2}} \mathcal{L}\left(\begin{array}{c}
\frac{\partial}{\partial \lambda_{1}} h \\
\frac{\partial}{\partial \lambda_{1}} p
\end{array}\right)\right\rangle\right|_{\lambda_{1}=\lambda_{2}=0} \\
& +\left(\int_{\infty}-\int_{B}\right)\left(\frac{\partial^{2}}{\partial \lambda_{1} \partial \lambda_{2}} Q_{t}-i_{t} \frac{\partial}{\partial \lambda_{2}} \theta\left(g ; \frac{\partial}{\partial \lambda_{1}} g\right)\right) .
\end{aligned}
$$

It is easily seen that the boundary contribution from infinity vanishes. Furthermore, the pullback to $B$ of $i_{t} \frac{\partial}{\partial \lambda_{2}} \theta\left(g ; \frac{\partial}{\partial \lambda_{1}} g\right)$ vanishes for $\lambda_{1}=\lambda_{2}=0$, since $t^{a}$ is tangent to $B$. Thus, we obtain

$$
\mathcal{E}\left(\gamma_{1}, \gamma_{2}\right)=\left\langle t \mid \frac{\partial}{\partial \lambda_{2}} \mathcal{L}\left(\begin{array}{c}
\frac{\partial}{\partial \lambda_{1}} h \\
\frac{\partial}{\partial \lambda_{1}} p
\end{array}\right)\right\rangle-\int_{B} \frac{\partial^{2}}{\partial \lambda_{1} \partial \lambda_{2}} Q_{t},
$$


where evaluation at $\lambda_{1}=\lambda_{2}=0$ is understood again. On the other hand, we have

$$
\left.\left\langle t \mid \frac{\partial}{\partial \lambda_{2}} \mathcal{L}\left(\begin{array}{c}
\frac{\partial}{\partial \lambda_{1}} h \\
\frac{\partial}{\partial \lambda_{1}} p
\end{array}\right)\right\rangle\right|_{\lambda_{1}=\lambda_{2}=0}=\left.\frac{\partial^{2}}{\partial \lambda_{1} \partial \lambda_{2}} \int_{\Sigma} t^{a} C_{a}\left(g+\lambda_{1} \delta_{1} g+\lambda_{2} \delta_{2} g\right)\right|_{\lambda_{1}=\lambda_{2}=0} .
$$

Since $t^{a} C_{a}=-\frac{1}{8 \pi} G_{a b} \nu^{a} t^{b} h^{\frac{1}{2}}$ the right side may be computed by taking second order variations of the Einstein tensor. This gives

$$
\mathcal{E}=\mathcal{E}(\gamma, \gamma)=-\left.\frac{1}{8 \pi} \int_{\Sigma} h^{\frac{1}{2}} \frac{d^{2}}{d \lambda^{2}} G_{a b}(g+\lambda \gamma) \nu^{a} t^{b}\right|_{\lambda=0}-\left.\int_{B} \frac{d^{2}}{d \lambda^{2}} Q_{t}(g+\lambda \gamma)\right|_{\lambda=0}
$$

Explicit evaluation of the two terms on the right side (see [20] for the first term, and use (9), (12), (33), (5) for the second term) gives:

$$
\begin{aligned}
\mathcal{E}= & \frac{1}{8 \pi} \int_{\Sigma} h^{\frac{1}{2}}\left(\gamma^{c d} \nabla_{a} \nabla_{b} \gamma_{c d}-2 \gamma^{c d} \nabla_{c} \nabla_{(a} \gamma_{b) d}+\frac{1}{2}\left(\nabla_{a} \gamma_{c d}\right) \nabla_{b} \gamma^{c d}+2\left(\nabla^{d} \gamma_{b}^{c}\right) \nabla_{[d} \gamma_{c] a}\right. \\
& \left.+\nabla_{d}\left(\gamma^{d c} \nabla_{c} \gamma_{a b}\right)-\frac{1}{2}\left(\nabla^{c} \gamma\right) \nabla_{c} \gamma_{a b}-2\left(\nabla_{d} \gamma^{c d}-\frac{1}{2} \nabla^{c} \gamma\right) \nabla_{(a} \gamma_{b) c}-\frac{1}{2} g_{a b}(\text { trace })\right) \nu^{a} t^{b} \\
& -\frac{\kappa}{16 \pi} \int_{B} \mu^{\frac{1}{2}} \delta \mu_{a b} \delta \mu^{a b}
\end{aligned}
$$

where $\delta \mu_{a b}$ is, as before, equal to the pullback of $\gamma_{a b}$ to $B$, where "trace" denotes the trace of the preceding terms, and where use has been made of the gauge conditions at $B$ in the computation of the boundary term. For later use, we also quote the lengthy expression for $\mathcal{E}$ in terms of the variables $\left(\delta h_{a b}, \delta p^{a b}\right)$ and the lapse and $\operatorname{shift}\left(N, N^{a}\right)$ of $t^{a}$, which can be obtained by taking a second variation of (68):

$$
\begin{aligned}
\mathcal{E}= & \frac{1}{16 \pi} \int_{\Sigma} N\left(h ^ { \frac { 1 } { 2 } } \left\{\frac{1}{2} R_{a b}(h) \delta h_{c}{ }^{c} \delta h^{a b}-2 R_{a c}(h) \delta h^{a b} \delta h_{b}{ }^{c}-\frac{1}{2} \delta h^{a c} D_{a} D_{c} \delta h_{d}{ }^{d}-\right.\right. \\
& \frac{1}{2} \delta h^{a c} D^{b} D_{b} \delta h_{a c}+\delta h^{a c} D^{b} D_{a} \delta h_{c b}-\frac{3}{2} D_{a}\left(\delta h^{b c} D^{a} \delta h_{b c}\right)-\frac{3}{2} D_{a}\left(\delta h^{a b} D_{b} \delta h_{c}{ }^{c}\right)+ \\
& \left.\frac{1}{2} D_{a}\left(\delta h_{d}{ }^{d} D^{a} \delta h_{c}{ }^{c}\right)+2 D_{a}\left(\delta h^{a}{ }_{c} D_{b} \delta h^{c b}\right)+D_{a}\left(\delta h^{b}{ }_{c} D_{b} \delta h^{a c}\right)-\frac{1}{2} D^{a}\left(\delta h_{c}{ }^{c} D^{b} \delta h_{a b}\right)\right\}+ \\
& h^{-\frac{1}{2}}\left\{2 \delta p_{a b} \delta p^{a b}+\frac{1}{2} p_{a b} p^{a b}\left(\delta h_{a}{ }^{a}\right)^{2}-p_{a b} \delta p^{a b} \delta h_{c}{ }^{c}-3 p^{a}{ }_{b} p^{b c} \delta h_{d}{ }^{d} \delta h_{a c}-\right. \\
& \frac{2}{D-2}\left(\delta p_{a}{ }^{a}\right)^{2}+\frac{3}{D-2} p_{c}{ }^{c} \delta p_{b}{ }^{b} \delta h_{a}{ }^{a}+\frac{3}{D-2} p_{d}{ }^{d} p^{a b} \delta h_{c}{ }^{c} \delta h_{a b}+8 p^{c} \delta h_{a c} \delta p^{a b}+ \\
& p_{c d} p^{c d} \delta h_{a b} \delta h^{a b}+2 p^{a b} p^{d c} \delta h_{a c} \delta h_{b d}-\frac{1}{D-2}\left(p_{c}{ }^{c}\right)^{2} \delta h_{a b} \delta h^{a b}-\frac{1}{2(D-2)}\left(p_{b}{ }^{b}\right)^{2}\left(\delta h_{a}{ }^{a}\right)^{2}- \\
& \left.\left.\frac{4}{D-2} p_{c}{ }^{c} \delta p^{a b} \delta h_{a b}-\frac{2}{D-2}\left(p^{a b} \delta h_{a b}\right)^{2}-\frac{4}{D-2} p_{a b} \delta p_{c}{ }^{c} \delta h^{a b}\right\}\right)- \\
& \frac{1}{16 \pi} \int_{\Sigma} N^{a}\left(-2 \delta p^{b c} D_{a} \delta h_{b c}+4 \delta p^{c b} D_{b} \delta h_{a c}+2 \delta h_{a c} D_{b} \delta p^{c b}-\right. \\
& \left.2 p^{c b} \delta h_{a d} D_{b} \delta h_{c}{ }^{d}+p^{c b} \delta h_{a d} D^{d} \delta h_{c b}\right)+\frac{\kappa}{16 \pi} \int_{B} \mu^{\frac{1}{2}}\left(\delta \mu_{a b} \delta \mu^{a b}-\frac{1}{2} \delta \mu_{a}{ }^{a} \delta \mu_{b}{ }^{b}\right) .
\end{aligned}
$$




\section{Flux of canonical energy at infinity and the horizon}

As shown in the previous section, canonical energy $\mathcal{E}(\gamma)$ is conserved for all solutions $\gamma_{a b}$ of the linearized Einstein equation in the sense that it is independent of the choice of Cauchy surface $\Sigma$ extending from spatial infinity to $B$. However, if we evaluate $\mathcal{E}$ on a slice $\mathscr{S}(t)$ that extends from a cross-section, $C(t)$, of future null infinity $\mathscr{I}^{+}$, (rather than from spatial infinity) to a cross section, $B(t)$, of the future horizon, $\mathscr{H}^{+}$, (rather than to $B)$

$$
\mathcal{E}(\gamma, \mathscr{S}(t)) \equiv W_{\mathscr{S}(t)}\left(\gamma, \mathscr{L}_{t} \gamma\right)=\int_{\mathscr{S}(t)} \omega\left(g ; \gamma, \mathscr{L}_{t} \gamma\right)
$$

then, of course, in general we will find that $\mathcal{E}(\gamma, \mathscr{S}(t)) \neq \mathcal{E}(\gamma)$. In this section we study the time evolution of $\mathcal{E}(\gamma, \mathscr{S}(t))$ as we march the slice $\mathscr{S}(t)$ forward in $t$. We will show that, up to boundary terms, $\mathcal{E}(\gamma, \mathscr{S}(t))$ decreases with $t$.

In order to be able to make use of the machinery of null infinity, we will restrict consideration in this section to even dimensional spacetimes- $\mathscr{I}$ does not exist for odd dimensional spacetimes [23]. However, we do not believe that this is an essential restriction, i.e., we believe that our results hold in odd dimensions, with a suitable notion of asymptotic flatness for that case, see e.g. [37].

We work in the "Bondi gauge" for the background metric $g_{a b}$, so the unphysical background metric $\tilde{g}_{a b}=\Omega^{2} g_{a b}$ near $\mathscr{I}^{+}$takes the form

$$
\tilde{g}_{a b}=2 \tilde{\nabla}_{(a} \Omega \tilde{\nabla}_{b)} \tilde{u}+\tilde{\mu}_{a b}+O(\Omega)
$$

with $\tilde{u}$ a future directed affine parameter on the null geodesic generators of $\mathscr{I}^{+}$. On $\mathscr{I}^{+}$ we have $(\partial / \partial \tilde{u})^{a}=\tilde{g}^{a b} \tilde{\nabla}_{b} \Omega \equiv \tilde{n}^{a}$, and $\tilde{\mu}_{a b}$ is the unit round metric on the $S^{D-2}$ crosssections of $\mathscr{I}^{+}$, with $\tilde{\mu}_{a b} \tilde{n}^{a}=0=\tilde{\mu}_{a b}(\partial / \partial \Omega)^{a}$. The asymptotically timelike Killing field $t^{a}$ can be extended continuously to a vector field $\tilde{t}^{a}$ on $\mathscr{I}^{+}$which is proportional to $\tilde{n}^{a}$, where we consequently have

$$
\tilde{t}^{a}=\left(\tilde{t}^{c} \tilde{\nabla}_{c} \tilde{u}\right) \tilde{n}^{a}
$$

with $\tilde{t}^{c} \tilde{\nabla}_{c} \tilde{u}>0$ and constant on $\mathscr{I}$. If the initial data for $\gamma_{a b}$ on $\Sigma$ are of compact support21, then it has been shown in 22] that there exists a gauge22 near future null infinity in which $\gamma_{a b}$ is asymptotically flat at future null-infinity $\mathscr{I}^{+}$in the sense that

$$
\tilde{\gamma}_{a b}:=\Omega^{-(D-6) / 2} \gamma_{a b}
$$

is smooth on $\mathscr{I}^{+}$, and that $\tilde{\gamma}_{a b} \tilde{g}^{a b}=O(\Omega), \tilde{\gamma}_{a b} \tilde{n}^{a}=O(\Omega)$.

It is also assumed throughout this section that $\gamma_{a b}$ is axisymmetric with respect to all of the axial Killing fields appearing in (12)

$$
\mathscr{L}_{\psi_{A}} \gamma_{a b}=0 \text { for all } A=1, \ldots, N \text {. }
$$

This restriction is essential because although we will obtain positivity of flux results at $\mathscr{I}^{+}$for canonical energy, we will obtain positivity of flux results at $\mathscr{H}^{+}$for the analogous

\footnotetext{
${ }^{21}$ Below, we will consider perturbations whose initial data are in a space $\mathcal{T}$. Elements in this space may not have compact support, but it is shown in lemma 5 of Appendix $\mathrm{C}$ that the initial data of compact support are dense in this space.

${ }^{22}$ In $D=4$, this is the Geroch-Xanthopoulos gauge, whereas in even $D>4$, it is the transverse-traceless gauge, see 22 for further details and other subtle differences between $D=4$ and the rest.
} 
quantity defined by replacing $t^{a}$ by the horizon Killing field $K^{a}$. It is only in the presence of axisymmetry (91) that these quantities are equal.

Let $\mathscr{I}_{12}$ denote the portion of future null-infinity bounded by cross-sections $C\left(t_{1}\right)$ and $C\left(t_{2}\right)$, and let $\mathscr{H}_{12}$ the portion of the horizon bounded by the cross-sections $B\left(t_{1}\right)$ and $B\left(t_{2}\right)$, see the following figure.

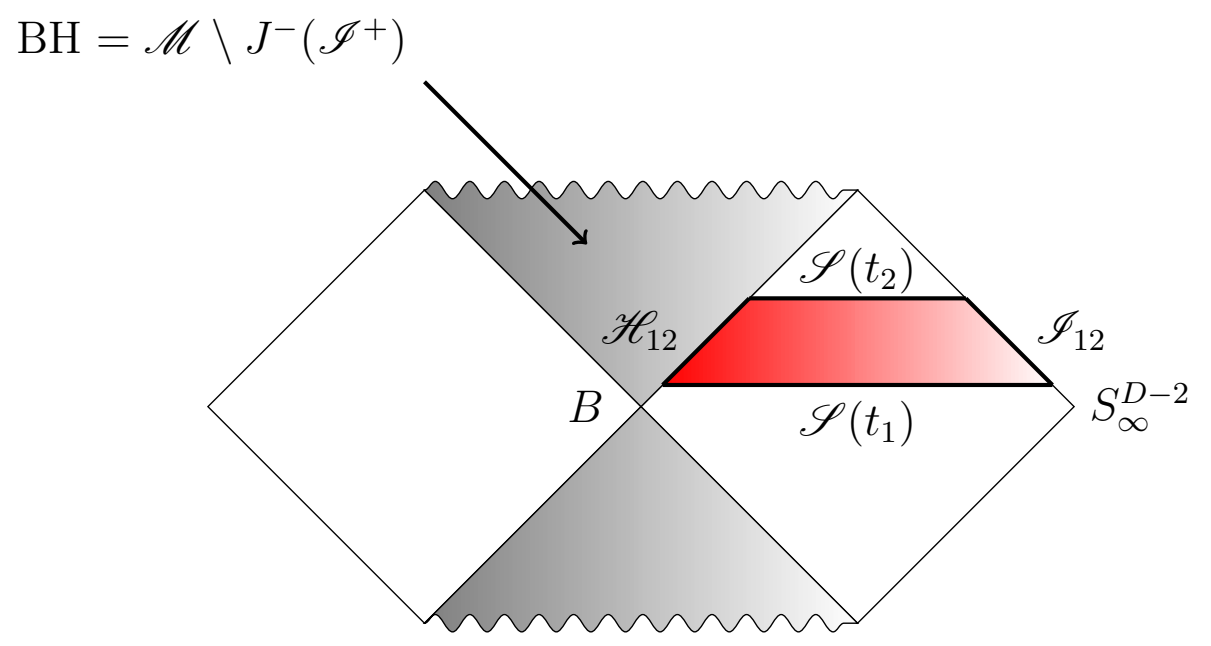

Clearly

$$
\mathscr{S}\left(t_{1}\right) \cup \mathscr{H}_{12} \cup \mathscr{I}_{12} \cup \mathscr{S}\left(t_{2}\right)
$$

bounds a compact region of the conformally compactified spacetime. By conservation of canonical energy, we have

$$
\mathcal{E}\left(\gamma, \mathscr{S}\left(t_{2}\right)\right)=\mathcal{E}\left(\gamma, \mathscr{S}\left(t_{1}\right)\right)-W_{\mathscr{I}_{12}}\left(\gamma, \mathscr{L}_{t} \gamma\right)-W_{\mathscr{H}_{12}}\left(\gamma, \mathscr{L}_{t} \gamma\right)
$$

Thus, $\mathcal{E}\left(\gamma, \mathscr{S}\left(t_{2}\right)\right)$ differs from $\mathcal{E}\left(\gamma, \mathscr{S}\left(t_{1}\right)\right)$ by the "flux terms" $W_{\mathscr{I}_{12}}\left(\gamma, \mathscr{L}_{t} \gamma\right)$ and $W_{\mathscr{H}_{12}}\left(\gamma, \mathscr{L}_{t} \gamma\right)$, corresponding, respectively, to the canonical energy radiated to null infinity and into the black hole.

We now evaluate $W_{\mathscr{I}_{12}}\left(\gamma, \mathscr{L}_{t} \gamma\right)$. Using a formula from [22] based on the Einstein equations for the background and perturbations, we have

$$
W_{\mathscr{I}_{12}}\left(\gamma_{1}, \gamma_{2}\right)=\frac{1}{32 \pi} \int_{\mathscr{I}_{12}}\left(\tilde{\gamma}_{1 c d} \delta_{2} \tilde{N}^{c d}-\tilde{\gamma}_{2 c d} \delta_{1} \tilde{N}^{c d}\right)
$$

where here and in the following, the natural integration measure on $\mathscr{I}^{+}$coming from $\tilde{g}_{a b}$ is understood 23 , and where indices on tilde tensor fields are always raised and lowered with $\tilde{g}_{a b}$. The Bondi news tensor is defined by ${ }^{24}$

$$
\tilde{N}_{c d}=\tilde{\mu}_{c}^{a} \tilde{\mu}_{d}^{b} \Omega^{-\frac{D-4}{2}}\left[\frac{2}{D-2} \tilde{R}_{a b}-\frac{1}{(D-1)(D-2)} \tilde{R} \tilde{g}_{a b}\right]-\frac{1}{D-2} \tilde{\mu}_{c d}(\text { trace })
$$

\footnotetext{
${ }^{23}$ In local coordinates $\Omega, u, x_{1}, \ldots, x_{D-2}$ adapted to (88), this would be $\tilde{\mu}^{\frac{1}{2}} \mathrm{~d} u \mathrm{~d} x_{1} \cdots \mathrm{d} x_{D-2}$.

${ }^{24}$ The form of the second term depends in general on the the conformal factor chosen near $\mathscr{I}$; the trace-type form is consistent with our gauge in which the cross sections of $\mathscr{I}$ are round spheres relative to the unphysical metric.
} 
where "trace" denotes the contraction with $\tilde{g}^{c d}$ of the preceding terms. As shown in [22], both $N_{a b}$ and $\delta \tilde{N}_{a b}=\frac{d}{d \lambda} \tilde{N}_{a b}(0)$ are smooth at $\mathscr{I}$ in our gauge. In fact, eq. (56) from [22], which uses the linearized Einstein equations, gives that, at $\mathscr{I}^{+}$,

$$
\delta \tilde{N}_{a b}=-\mathscr{L}_{\tilde{n}} \tilde{\gamma}_{a b}+D \Omega^{-1} \tilde{n}_{(a} \tilde{\gamma}_{b) c} \tilde{n}^{c}-\frac{D-2}{2} \Omega^{-1} \tilde{n}^{c} \tilde{n}_{c} \tilde{\gamma}_{a b}
$$

From this, eq. (89), and using also $\tilde{n}^{c} \tilde{n}_{c}=O\left(\Omega^{2}\right)$ since $\tilde{u}$ is an affine parameter on scri, we obtain

$$
W_{\mathscr{I}_{12}}\left(\gamma, \mathscr{L}_{t} \gamma\right)=\frac{1}{16 \pi} \int_{\mathscr{I}_{12}}\left(\tilde{t}^{a} \tilde{\nabla}_{a} \tilde{u}\right) \delta \tilde{N}_{c d} \delta \tilde{N}^{c d}+\mathcal{C}\left(t_{2}\right)-\mathcal{C}\left(t_{1}\right)
$$

where

$$
\mathcal{C}(t):=\frac{1}{32 \pi} \int_{C(t)}\left(\tilde{t}^{a} \tilde{\nabla}_{a} \tilde{u}\right) \tilde{\gamma}^{c d}\left(\mathscr{L}_{\tilde{n}} \tilde{\gamma}_{c d}\right)
$$

and where the natural integration element on $C(t)$ induced by $\tilde{\mu}_{a b}$ is understood.

Next, we evaluate $W_{\mathscr{H}_{12}}\left(\gamma, \mathscr{L}_{t} \gamma\right)$. The Raychaudhuri equation on $\mathscr{H}^{+}$yields

$$
\frac{d}{d u} \vartheta(\lambda)=-\frac{1}{D-2} \vartheta(\lambda)^{2}-\sigma_{a b}(\lambda) \sigma^{a b}(\lambda)-R_{a b}(\lambda) n^{a} n^{b}
$$

where, as before,

$$
\vartheta=\frac{1}{2} \mu^{a b} \mathscr{L}_{n} \mu_{a b}
$$

denotes the expansion of the generators of $\mathscr{H}^{+}$, whereas $\sigma_{a b}$ denotes their shear

$$
\frac{1}{2} \mathscr{L}_{n} \mu_{a b}=\sigma_{a b}+\frac{1}{D-2} \mu_{a b} \vartheta
$$

Here, the quantities $\mu_{a b}$ and $n^{a}=(\partial / \partial u)^{a}$ refer to the horizon metric; see (5). We take a $\lambda$-derivative of this equation and evaluate at $\lambda=0$. Then, since $\vartheta(0)=0=\sigma_{a b}(0)$ for the background metric $g_{a b}(0)$, and since $\delta R_{a b}=d R_{a b} / d \lambda(0)=0$ by the linearized Einstein equations, we have $d \delta \vartheta / d u=0$. Since $\delta \vartheta(0)=0$ on $B$ by our choice of gauge (see subsection 2.1), it follows that $\delta \vartheta=0$ on the entire horizon, as we have previously claimed. This implies that $\mathscr{L}_{n}\left(g^{a b} \gamma_{a b}\right)=0$. Using this fact, one can show that

$$
W_{\mathscr{H}_{12}}\left(\gamma, \mathscr{L}_{t} \gamma\right)=\frac{1}{32 \pi} \int_{\mathscr{H}_{12}}\left(\mathscr{L}_{K} \gamma^{c d} \mathscr{L}_{n} \gamma_{c d}-\gamma^{c d} \mathscr{L}_{n} \mathscr{L}_{K} \gamma_{c d}\right)
$$

where we have used the axisymmetry (91) of $\gamma_{a b}$ to replace $\mathscr{L}_{t} \gamma_{c d}$ by $\mathscr{L}_{K} \gamma_{c d}$, where $K^{a}$ is the Killing field (12) normal to the horizon. Using $K^{a}=\kappa u n^{a}$, we obtain

$$
W_{\mathscr{H}_{12}}\left(\gamma, \mathscr{L}_{t} \gamma\right)=\frac{1}{4 \pi} \int_{\mathscr{H}_{12}}\left(K^{a} \nabla_{a} u\right) \delta \sigma_{c d} \delta \sigma^{c d}+\mathcal{B}\left(t_{2}\right)-\mathcal{B}\left(t_{1}\right)
$$

where

$$
\mathcal{B}(t) \equiv \frac{1}{32 \pi} \int_{B(t)}\left(K^{a} \nabla_{a} u\right) \gamma^{c d}\left(\mathscr{L}_{n} \gamma_{c d}\right)
$$


and where the natural integration element on $B(t)$ induced by $\mu_{a b}$ is understood. The above calculations motivate the definition of a modified canonical energy $\overline{\mathcal{E}}(\gamma, \mathscr{S}(t))$ given by

$$
\begin{aligned}
& \overline{\mathcal{E}}(\gamma, \mathscr{S}(t)):=\mathcal{E}(\gamma, \mathscr{S}(t)) \\
& -\frac{1}{32 \pi} \int_{C(t)}\left(\tilde{t}^{a} \tilde{\nabla}_{a} \tilde{u}\right) \tilde{\gamma}^{c d}\left(\mathscr{L}_{\tilde{n}} \tilde{\gamma}_{c d}\right)-\frac{1}{32 \pi} \int_{B(t)}\left(K^{a} \nabla_{a} u\right) \gamma^{c d}\left(\mathscr{L}_{n} \gamma_{c d}\right)
\end{aligned}
$$

Thus, the modified canonical energy $\overline{\mathcal{E}}(\gamma, \mathscr{S}(t))$ differs from $\mathcal{E}(\gamma, \mathscr{S}(t))$ only by the above boundary terms $\mathcal{C}(t)$ and $\mathcal{B}(t)$. Note that $\mathcal{C}(t)$ vanishes when the perturbed Bondi news, $\delta N_{a b}$, vanishes. This is seen using eq. (96), the fact [22] that $\tilde{\gamma}_{a b} \tilde{n}^{a}=O(\Omega)$, and that $\tilde{n}^{a} \tilde{n}_{a}=O\left(\Omega^{2}\right)$, which in turn follows since $\tilde{u}$ is by construction an affine parameter in $\mathscr{I}^{+}$. Also, $\mathcal{B}(t)$ vanishes when the perturbed shear, $\delta \sigma_{a b}$, vanishes, since $\delta \sigma_{a b}=\frac{1}{2} \mathscr{L}_{n} \gamma_{a b}$ on $\mathscr{H}^{+}$, by (101), $\delta \vartheta=0=\vartheta$ on $\mathscr{H}^{+}$, and by expression eq. (149) for $\gamma_{a b}$. In addition, $\mathcal{B}$ vanishes at $B$ since $\left.K^{a}\right|_{B}=0$. Since the perturbed Bondi news vanishes as one approaches spatial infinity 25 it follows that $\overline{\mathcal{E}}(\gamma, \mathscr{S}(t)) \rightarrow \mathcal{E}(\gamma, \Sigma)$ as $\mathscr{S}(t) \rightarrow \Sigma$.

The above results establish the following theorem:

Theorem 1. Let $\gamma_{a b}$ be an axisymmetric linearized perturbation that, in addition to the properties assumed in the previous section, is asymptotically flat at future null infinity. Then the modified canonical energy (105) has the property that, for $t_{1}<t_{2}$

$$
\begin{aligned}
& \overline{\mathcal{E}}\left(\gamma, \mathscr{S}\left(t_{2}\right)\right)-\overline{\mathcal{E}}\left(\gamma, \mathscr{S}\left(t_{1}\right)\right) \\
& =-\frac{1}{16 \pi} \int_{\mathscr{I}_{12}}\left(\tilde{t}^{a} \tilde{\nabla}_{a} \tilde{u}\right) \delta \tilde{N}_{c d} \delta \tilde{N}^{c d}-\frac{1}{4 \pi} \int_{\mathscr{H}_{12}}\left(K^{a} \nabla_{a} u\right) \delta \sigma_{c d} \delta \sigma^{c d} \leq 0 .
\end{aligned}
$$

We remark that Habisohn [20] has considered, in $D=4$ dimensions, the second order Einstein tensor, and has derived a balance law for the flux through an infinitely extended timelike tube approaching null infinity that is similar to that given above in (106), although his balance law does not refer to the flux between fixed times $t_{1}$ and $t_{2}$ as above, but to the total flux for all times.

\section{$4 \quad$ Stability and instability}

As seen is section 2, the canonical energy, $\mathcal{E}$, can be viewed as a quadratic form defined on the vector space of smooth, linearized solutions that satisfy our asymptotic conditions at spatial infinity and our gauge conditions near the horizon (see subsection 2.1). If $\mathcal{E}$ were positive definite on this vector space, it would provide a conserved norm that could be used to argue for stability. On the other hand, if $\mathcal{E}<0$ for some perturbation, then the flux results of the previous section show that the modified canonical energy $\overline{\mathcal{E}}$ on the slice $\mathscr{S}(t)$ can only become more negative with time, which suggests instability. However, there are a number of obvious difficulties with making such arguments. In particular:

- As already noted [see ([56) $], \mathcal{E}$ is negative for the "change of mass" perturbation of the Schwarzschild black hole, yet Schwarzschild is known to be linearly stable. Such "trivial" perturbations must be eliminated from the analysis.

25 This is manifestly true because we consider perturbations having compact support on $\Sigma$; see footnote 21 
- $\mathcal{E}$ will, in general, be degenerate on some perturbations, so, even if positive, it cannot be expected to be positive definite. Thus, the degeneracies of $\mathcal{E}$ must be carefully analyzed.

In order to properly analyze the degeneracies of $\mathcal{E}$, it will be very useful to view $\mathcal{E}$ as a densely defined quadratic form on a Hilbert space. In the next subsection, we will introduce such a Hilbert space $\mathcal{V}$-whose construction and properties may be of some interest in their own right-and analyze the degeneracies of $\mathcal{E}$. It should be noted that the perturbations in $\mathcal{V}$ will have vanishing linearized ADM mass, linear momentum, and angular momentum with respect to rotational Killing fields, $\delta M=\delta P_{i}=\delta J_{A}=0$, so they will automatically eliminate the "trivial" perturbations corresponding to variations of the mass or angular momentum in a family of stationary black holes. We will then make our stability/instability arguments in subsection 4.2.

\subsection{The Hilbert space $\mathcal{V}$ and the degeneracies of $\mathcal{E}$}

We start26 with the real Hilbert space

$$
\mathcal{K}=L^{2}\left(\Sigma,\left(T^{*} \Sigma\right)^{\vee 2} ; h^{\frac{1}{2}} e_{0}\right) \oplus L^{2}\left(\Sigma,(T \Sigma)^{\vee 2} \otimes \Lambda^{\frac{1}{2}} ; h^{-\frac{1}{2}} e_{0}\right)
$$

of all square integrable linearized initial data, not necessarily satisfying the constraints. Here $\Lambda^{\frac{1}{2}}$ is the line bundle over $\Sigma$ of densities of weight one half. Thus, the first summand denotes the Hilbert space of square integrable symmetric tensors on $\Sigma$, whereas the second denotes the Hilbert space of square integrable symmetric tensor densities of weight $\frac{1}{2}$ over $\Sigma$. In other words, elements of $\mathcal{K}$ consist of pairs $\left(\delta h_{a b}, \delta p^{a b}\right)$ with inner product

$$
\left\langle\left(\begin{array}{l}
\delta_{1} h \\
\delta_{1} p
\end{array}\right) \mid\left(\begin{array}{l}
\delta_{2} h \\
\delta_{2} p
\end{array}\right)\right\rangle_{\mathcal{K}}:=\int_{\Sigma} h^{\frac{1}{2}} \delta_{1} h_{a b} \delta_{2} h^{a b}+h^{-\frac{1}{2}} \delta_{1} p_{a b} \delta_{2} p^{a b} .
$$

Note that for $D=4,5$, the requirement of square integrability will impose faster fall-off conditions at infinity on $\delta h_{a b}$ (but not $\delta p^{a b}$ ) than assumed in section 2 and will exclude the possibility of having a nonvanishing $\delta M$, but for $D \geq 6, \mathcal{K}$ will include all smooth initial data satisfying the asymptotic conditions at spatial infinity stated at the beginning of subsection 2.1. We may view the symplectic form (28) as a bounded linear map $S: \mathcal{K} \rightarrow \mathcal{K}$ defined by 27

$$
16 \pi W_{\Sigma}\left(\delta_{1} g, \delta_{2} g\right)=\left\langle\left(\begin{array}{c}
\delta_{1} h \\
\delta_{1} p
\end{array}\right) \mid S\left(\begin{array}{c}
\delta_{2} h \\
\delta_{2} p
\end{array}\right)\right\rangle_{\mathcal{K}}
$$

where

$$
S \equiv\left(\begin{array}{cc}
0 & -h^{-\frac{1}{2}} h_{a c} h_{b d} \\
h^{\frac{1}{2}} h^{a c} h^{b d} & 0
\end{array}\right)
$$

\footnotetext{
${ }^{26}$ Although we will restrict consideration to axisymmetric perturbations when we make our stability arguments, the constructions of this subsection do not require axisymmetry, and we will not impose this restriction until the end of this subsection.

${ }^{27}$ Note that, in subsec. 2.4 the angles $\langle\mid\rangle$ as e.g. in (72) denote the dual pairing between tensors and densities, whereas $\langle\mid\rangle_{\mathcal{K}}$ is an inner product between objects of the same density. As a consequence, the definition of $\mathcal{L}^{*}$, defined relative to $\langle\mid\rangle_{\mathcal{K}}$, used from now on, differs from the earlier one in (74) by a factor of $\operatorname{diag}\left(h^{-\frac{1}{2}}, h^{\frac{1}{2}}\right)$. This also accounts for the difference between $S$ and $\sigma$ in (77).
} 
Note that $S^{*}=-S$ and $S^{2}=-I$, so, in particular, $S$ is an orthogonal map, $S^{*} S=I$.

We would now like to pass to the subspace of $\mathcal{K}$ that satisfies the constraints and satisfies our gauge conditions. At first sight, it might appear that this would be a difficult task, given that generic elements of $\mathcal{K}$ are not even differentiable. However, the desired subspace $\mathcal{V}$ can be defined straightforwardly as follows: Let

$$
\begin{aligned}
\mathcal{W}:= & \left\{\mathscr{L}_{X} g_{a b} \in \mathcal{K} \mid X^{a} \in C^{\infty}, \text { near infinity } X^{a}\right. \text { coincides with a rotational Killing } \\
& \text { field plus an asymptotic translation, } \left.\left.X^{a}\right|_{B} \text { tangent to generators of } \mathscr{H}^{+}\right\}(110)
\end{aligned}
$$

Here, by $\mathscr{L}_{X} g_{a b}$ we mean the corresponding initial data $\left(\left(\mathscr{L}_{X} g\right)_{h},\left(\mathscr{L}_{X} g\right)_{p}\right)$. The space of interest for us is the space $\mathcal{V}$ of initial data symplectically orthogonal to $\mathcal{W}$ :

$$
\mathcal{V}=\mathcal{W}^{\perp_{S}}=\left\{(\delta h, \delta p) \in \mathcal{K}:\left\langle\left(\begin{array}{l}
\delta h \\
\delta p
\end{array}\right) \mid S\left(\begin{array}{l}
\delta h^{\prime} \\
\delta p^{\prime}
\end{array}\right)\right\rangle_{\mathcal{K}}=0 \quad \text { for all }\left(\delta h^{\prime}, \delta p^{\prime}\right) \in \mathcal{W}\right\}
$$

Thus, $\mathcal{V}$ is a closed subspace of $\mathcal{K}$ and, thus, is itself a Hilbert space. Note that since $S^{*}=-S$, we have $\mathcal{V}^{\perp_{S}}=\overline{\mathcal{W}}$, where $\overline{\mathcal{W}}$ denotes the closure of $\mathcal{W}$.

In the following, we will need to work with tensor fields that are in the weighted Sobolev spaces $W_{\rho}^{k} \equiv W_{\rho}^{k}(\Sigma)$. These spaces denote the weakly differentiable tensor fields $u$ on $\Sigma$ such that $\rho^{n} D_{\left(a_{1}\right.} \ldots D_{\left.a_{n}\right)} u \in L^{2}$ for all $n \leq k$, where $\rho>0$ is a smooth function on $\Sigma$ which interpolates between 1 in a neighborhood of $B$ and $\left(x_{1}^{2}+\cdots+x_{D-1}^{2}\right)^{\frac{1}{2}}$ in a neighborhood of infinity. The weight factor $\rho^{n}$ for the $n$-th derivative is inserted so as to force weak derivatives to fall off faster by an appropriate power. The weighted Sobolev norm for $u \in W_{\rho}^{k}$ is defined by

$$
\|u\|_{W_{\rho}^{k}}:=\left\{\sum_{n=0}^{k} \int_{\Sigma} h^{\frac{1}{2}} \rho^{2 n}\left(D_{\left(a_{1}\right.} \cdots D_{\left.a_{n}\right)} u\right) D^{\left(a_{1}\right.} \cdots D^{\left.a_{n}\right)} u\right\}^{\frac{1}{2}} .
$$

We will use the notation $C_{0}^{\infty}(\Sigma)$ to denote tensor fields that are smooth and of compact support on $\Sigma \backslash B$. We will use the notation $C^{\infty}(\bar{\Sigma})$ to denote smooth tensor fields on $\Sigma$ that can be smoothly extended across $B$.

We denote by $\mathcal{U}$ the space

$$
\mathcal{U}=\bigcap_{k}\left(W_{\rho}^{k}(\Sigma) \oplus W_{\rho}^{k}(\Sigma)\right)
$$

Clearly, $\mathcal{U}$ is a subspace of $\mathcal{K}$, and it follows from the Sobolev embedding theorems that $\mathcal{U} \subset C^{\infty}(\bar{\Sigma}) \oplus C^{\infty}(\bar{\Sigma})$.

The proof of property (3) of proposition 5 below will require the following lemma:

Lemma 3. Let $Y=\left(N_{0}, N_{0}^{a}\right) \in C_{0}^{\infty} \oplus C_{0}^{\infty}$. Then there exists a solution $X$ in the space $\cap_{k}\left(\rho^{2} W_{\rho}^{k+1}(\Sigma) \oplus \rho W_{\rho}^{k}(\Sigma)\right) \subset C^{\infty}(\bar{\Sigma}) \oplus C^{\infty}(\bar{\Sigma})$ to the following boundary value problem:

$$
\mathcal{L} \mathcal{L}^{*}(X)=Y \quad \text { in } \Sigma,
$$

and $X=\left(N, N^{a}\right)$ satisfies

$$
N^{a}=N \eta^{a}, \quad \delta \vartheta=\delta \epsilon=0 \quad \text { on } B=\partial \Sigma .
$$


Here $\mathcal{L}$ and $\mathcal{L}^{*}$ are given by (70) and (74) and $\delta \vartheta, \delta \epsilon$ are the perturbed expansion/volume element on $B$ associated with the perturbation $(\delta h, \delta p)=\mathcal{L}^{*}(X)$, given by

$$
\begin{aligned}
\left.\delta \vartheta\right|_{B}= & h^{-\frac{1}{2}}\left(h^{a b}-\eta^{a} \eta^{b}\right)\left(\delta p_{a b}-\frac{1}{2} \delta h_{c}^{c} p_{a b}+2 p_{c(a} \delta h_{b)}{ }^{c}+\frac{1}{2} h^{\frac{1}{2}} \mathscr{L}_{\eta} \delta h_{a b}\right. \\
& \left.-\frac{1}{D-2}\left(-\frac{1}{2} p h_{a b} \delta h_{c}^{c}+p^{c d} \delta h_{c d} h_{a b}+p \delta h_{a b}+\delta p_{c}{ }^{c} h_{a b}\right)\right)\left.\right|_{B} \\
\left.\delta \epsilon\right|_{B}= & \left.\frac{1}{2}\left(h^{a b}-\eta^{a} \eta^{b}\right) \delta h_{a b}\right|_{B},
\end{aligned}
$$

where $\eta^{a}$ is the unit inward normal to $B$ within $\Sigma$. Furthermore, if $D \geq 5$, the solution is unique, whereas for $D=4$, the solution is also unique unless $t^{a}$ is tangent to the generators of the horizon (i.e., the black hole is nonrotating), in which case the solution is unique up to $X^{a} \rightarrow X^{a}+c t^{a}$.

A proof of this lemma is given in appendix B.

The key properties of $\mathcal{V}$ are now summarized in the following proposition:

Proposition 5. 1. Let $(\delta h, \delta p) \in \mathcal{V}$. Then $(\delta h, \delta p)$ is a distributional solution to the linearized constraints in the sense that

$$
\left\langle\mathcal{L}^{*}(X) \mid\left(\begin{array}{l}
\delta h \\
\delta p
\end{array}\right)\right\rangle_{\mathcal{K}}=0,
$$

for all $X^{a} \in C_{0}^{\infty}$.

2. If $(\delta h, \delta p)$ is a smooth element of $\mathcal{V}$, then $\delta M=\delta J_{A}=\delta P_{i}=0$ and $\left.\delta \epsilon\right|_{B}=\left.\delta \vartheta\right|_{B}=$ 0 . Conversely, if $(\delta h, \delta p) \in \mathcal{K}$ is a smooth solution of the linearized constraints satisfying $\delta M=\delta J_{A}=\delta P_{i}=0$ and $\left.\delta \epsilon\right|_{B}=\left.\delta \vartheta\right|_{B}=0$, then $(\delta h, \delta p) \in \mathcal{V}$.

3. The space $\mathcal{U} \cap \mathcal{V}$ is dense in $\mathcal{V}$, where $\mathcal{U}$ was defined by (113).

Proof: Property (1) is an immediate consequence of (75) together with the fact that for any $X^{a} \in C_{0}^{\infty}$, we clearly have $\mathscr{L}_{X} g_{a b} \in \mathcal{W}$. Property (2) is an immediate consequence 28 of lemma 2 .

Our strategy for proving property (3) is to define a bounded projection operator $\Pi$ : $\mathcal{K} \rightarrow \mathcal{V}$ which takes $C_{0}^{\infty} \oplus C_{0}^{\infty} \subset L^{2} \oplus L^{2} \equiv \mathcal{K}$ to elements in $\mathcal{U} \cap \mathcal{V}$. Since $C_{0}^{\infty} \oplus C_{0}^{\infty}$ is dense in $\mathcal{K}$, it will then follow that $\mathcal{U} \cap \mathcal{V}$ is dense in $\mathcal{V}$. We will produce the desired operator $\Pi$ in two steps. First we define an orthogonal projector $\Pi_{0}: \mathcal{K} \rightarrow \mathcal{V}_{0}$ onto a closed subspace $\mathcal{V}_{0} \subset \mathcal{K} \equiv L^{2} \oplus L^{2}$ containing $\mathcal{V}$. The space $\mathcal{V}_{0}$ "imposes the constraints" and the "correct boundary conditions" $\left.\delta \epsilon\right|_{B}=\left.\delta \vartheta\right|_{B}=0$ at $B$, but does not impose $\delta M=\delta J_{A}=\delta P_{i}=0$. We will then compose $\Pi_{0}$ with a finite co-rank projection operator $\Pi_{1}$ that commutes with $\Pi_{0}$ to obtain the desired projection operator $\Pi$. Since orthogonal projectors and finite co-rank projectors are bounded, $\Pi$ is bounded.

Our prescription for the operator $\Pi_{0}$ is as follows. Let $\left(\delta h_{0}, \delta p_{0}\right) \in C_{0}^{\infty} \oplus C_{0}^{\infty}$. Then, obviously, $Y:=\mathcal{L}\left(\delta h_{0}, \delta p_{0}\right)$ is smooth and of compact support. Let $X$ be the solution to

$$
\mathcal{L} \mathcal{L}^{*}(X)=Y
$$

\footnotetext{
${ }^{28}$ Our asymptotic conditions at infinity are now that $(\delta h, \delta p) \in \mathcal{K}$ rather than the conditions that follow from those stated in the second paragraph of subsection 2.1. It is easily verified that the proof of lemma 2 continues to hold with our present asymptotic conditions.
} 
given by lemma 3 above. We define 29

$$
\Pi_{0}\left(\begin{array}{l}
\delta h_{0} \\
\delta p_{0}
\end{array}\right)=\left(\begin{array}{l}
\delta h_{0} \\
\delta p_{0}
\end{array}\right)-\mathcal{L}^{*}(X)
$$

It follows immediately that $\Pi_{0}\left(\delta h_{0}, \delta p_{0}\right) \in \mathcal{U}$, that it satisfies the linearized constraints, and that it satisfies $\left.\delta \vartheta\right|_{B}=0=\left.\delta \epsilon\right|_{B}$ [cf. eqs. (116) and (117)]. Since we have

$$
\begin{aligned}
& \left\|\mathcal{L}^{*}(X)\right\|_{\mathcal{K}}^{2}=\langle X \mid Y\rangle=\left\langle X \mid \mathcal{L}\left(\begin{array}{c}
\delta h_{0} \\
\delta p_{0}
\end{array}\right)\right\rangle= \\
& \left\langle\mathcal{L}^{*}(X) \mid\left(\begin{array}{c}
\delta h_{0} \\
\delta p_{0}
\end{array}\right)\right\rangle_{\mathcal{K}} \leq\left\|\mathcal{L}^{*}(X)\right\|_{\mathcal{K}}\left\|\left(\begin{array}{l}
\delta h_{0} \\
\delta p_{0}
\end{array}\right)\right\|_{\mathcal{K}}
\end{aligned}
$$

(where eq. (164) of Appendix B was used in the first equality) it follows that $\Pi_{0}$ is bounded and, hence, its action can be extended to $\mathcal{K}$. We now show that $\Pi_{0}=\Pi_{0}^{*}$ and $\Pi_{0}^{2}=\Pi_{0}$, so $\Pi_{0}$ is an orthogonal projection. To show that $\Pi_{0}^{*}=\Pi_{0}$, we let $\Psi_{0}:=\left(\delta h_{0}, \delta p_{0}\right)$ and $\Psi_{0}^{\prime}:=\left(\delta h_{0}^{\prime}, \delta p_{0}^{\prime}\right)$ be smooth and of compact support. Then

$$
\begin{aligned}
& \left\langle\Pi_{0} \Psi_{0} \mid \Psi_{0}^{\prime}\right\rangle_{\mathcal{K}}=\left\langle\Psi_{0} \mid \Psi_{0}^{\prime}\right\rangle_{\mathcal{K}}-\left\langle\mathcal{L}^{*}(X) \mid \Psi_{0}\right\rangle_{\mathcal{K}} \\
= & \left\langle\Psi_{0} \mid \Psi_{0}^{\prime}\right\rangle_{\mathcal{K}}-\left\langle X \mid Y^{\prime}\right\rangle_{\mathcal{K}}=\left\langle\Psi_{0} \mid \Psi_{0}^{\prime}\right\rangle_{\mathcal{K}}-\left\langle\mathcal{L}^{*}(X) \mid \mathcal{L}^{*}\left(X^{\prime}\right)\right\rangle_{\mathcal{K}}
\end{aligned}
$$

where $X, X^{\prime}, Y, Y^{\prime}$ are defined as above and, in the last step, we again used (164). The expression on the right is manifestly symmetric, thus proving the claim. Similarly, we have

$$
\begin{aligned}
\left\langle\Pi_{0} \Psi_{0} \mid \Pi_{0} \Psi_{0}^{\prime}\right\rangle_{\mathcal{K}} & =\left\langle\Psi_{0} \mid \Pi_{0} \Psi_{0}^{\prime}\right\rangle_{\mathcal{K}}-\left\langle\mathcal{L}^{*}(X) \mid \Pi_{0} \Psi_{0}^{\prime}\right\rangle_{\mathcal{K}} \\
& =\left\langle\Psi_{0} \mid \Pi_{0} \Psi_{0}^{\prime}\right\rangle_{\mathcal{K}}
\end{aligned}
$$

thus showing that $\Pi_{0}^{2}=\Pi_{0}$.

Let $\mathcal{V}_{0}$ denote the closure in $\mathcal{K}$ of the image of $C_{0}^{\infty} \oplus C_{0}^{\infty}$ under $\Pi_{0}$. We now show that $\mathcal{V} \subset \mathcal{V}_{0}$. Suppose that $(\delta h, \delta p) \in \mathcal{V}$ is orthogonal to $\Pi_{0}\left(C_{0}^{\infty} \oplus C_{0}^{\infty}\right)$. Then inserting (120), we see that

$$
0=\left\langle\left(\begin{array}{l}
\delta h \\
\delta p
\end{array}\right) \mid\left(\begin{array}{l}
\delta h_{0} \\
\delta p_{0}
\end{array}\right)\right\rangle_{\mathcal{K}}-\left\langle\left(\begin{array}{l}
\delta h \\
\delta p
\end{array}\right) \mid \mathcal{L}^{*}(X)\right\rangle_{\mathcal{K}} .
$$

The second term vanishes, because $X \in \rho^{2} W_{\rho}^{2} \oplus \rho W_{\rho}^{1}$, in which $C_{0}^{\infty}(\bar{\Sigma}) \oplus C_{0}^{\infty}(\bar{\Sigma})$ are dense. Then, from the boundary conditions of $X$ (see lemma 3 ), $X$ can be approximated by $X_{n} \in \mathcal{W}$ such that $\mathcal{L}^{*} X_{n} \rightarrow \mathcal{L}^{*} X$ in $L^{2} \oplus L^{2}$. Hence, since $(\delta h, \delta p)$ in $\mathcal{W}^{\perp_{S}}$, it follows that $\left\langle(\delta h, \delta p) \mid \mathcal{L}^{*}(X)\right\rangle_{\mathcal{K}}=0$. Thus, we see that $0=\left\langle(\delta h, \delta p) \mid\left(\delta h_{0}, \delta p_{0}\right)\right\rangle_{\mathcal{K}}$ for any compactly supported $\left(\delta h_{0}, \delta p_{0}\right)$, which clearly means that $(\delta h, \delta p)=0$. Thus, the closure in $\mathcal{K}$ of $\Pi_{0}\left(C_{0}^{\infty} \oplus C_{0}^{\infty}\right)$ contains $\mathcal{V}$.

On the other hand, since all elements $(\delta h, \delta p) \in \Pi_{0}\left(C_{0}^{\infty} \oplus C_{0}^{\infty}\right)$ are in $\mathcal{U}$ (and, hence, are smooth), satisfy the linearized constraints, and satisfy $\left.\delta \vartheta\right|_{B}=0=\left.\delta \epsilon\right|_{B}$, it follows from property (2) of this proposition that $(\delta h, \delta p) \in \mathcal{V}$ if and only if $\delta M=\delta J_{A}=\delta P_{i}=0$. Therefore, to obtain the desired projection map we need only compose $\Pi_{0}$ with a finite co-rank projector $\Pi_{1}$ defined as follows: Let $\xi_{I}^{a}, I=1, \ldots, k$ denote a finite collection of

\footnotetext{
${ }^{29}$ See footnote 27.
} 
smooth vector fields that vanish in a neighborhood of $B$ and coincide, respectively, with $t^{a}$, $\psi_{A}{ }^{a}$, and $\left(\partial / \partial x^{i}\right)^{a}$ in a neighborhood of infinity. Let $\Psi_{I}:=\left(\delta_{I} h, \delta_{I} p\right), i=1, \ldots, k$ be an (arbitrarily chosen) collection of perturbations in $\Pi_{0}\left(C_{0}^{\infty} \oplus C_{0}^{\infty}\right)$ for which $\delta H_{\xi_{I}}\left(\Psi_{J}\right)=\delta_{I J}$. The desired projector $\Pi_{1}$ is defined by

$$
\Pi_{1}\left(\begin{array}{l}
\delta h \\
\delta p
\end{array}\right)=\left(\begin{array}{l}
\delta h \\
\delta p
\end{array}\right)-\sum_{I=1}^{k}\left|\Psi_{I}\right\rangle\left\langle\left(\begin{array}{l}
\delta h \\
\delta p
\end{array}\right) \mid \mathcal{L}^{*}\left(\xi_{I}\right)\right\rangle_{\mathcal{K}}
$$

and the desired projector $\Pi$ onto $\mathcal{V}$ is then defined by $\Pi=\Pi_{1} \Pi_{0}$. By our arguments above, the image of $\Pi\left(C_{0}^{\infty} \oplus C_{0}^{\infty}\right)$ under $\Pi$ is contained in $\mathcal{U}$ and is dense in $\mathcal{V}$ thus proving that $\mathcal{U} \cap \mathcal{V}$ is dense in $\mathcal{V}$.

Remark 1: It is possible to strengthen property (3) using gluing techniques to show that even $\left(C_{0}^{\infty}(\bar{\Sigma}) \oplus C_{0}^{\infty}(\bar{\Sigma})\right) \cap \mathcal{V}$ is dense in $\mathcal{V}$, where by definition $C_{0}^{\infty}(\bar{\Sigma}) \oplus C_{0}^{\infty}(\bar{\Sigma}) \subset \mathcal{U}$ consists of all smooth initial data that vanishes in a neighborhood of spatial infinity. A statement and proof of this strengthened result is given in Appendix C.

Remark 2: All elements of $\mathcal{W}$ are smooth solutions to the linearized constraints and satisfy $\delta M=\delta J_{A}=\delta P_{i}=0$ and $\left.\delta \epsilon\right|_{B}=\left.\delta \vartheta\right|_{B}=0$. Thus, by property (2) of the above Proposition, we have $\mathcal{W} \subset \mathcal{V}$ and, hence, $\overline{\mathcal{W}} \subset \mathcal{V}$. On the other hand, since $\overline{\mathcal{W}}$ and $\mathcal{V}$ are symplectic complements of each other in $\mathcal{K}$, it follows immediately that an element $\gamma=(\delta h, \delta p) \in \mathcal{V}$ is such that $W_{\Sigma}\left(g ; \gamma^{\prime}, \gamma\right)=0$ for all $\gamma^{\prime} \in \mathcal{V}$ if and only if $\gamma \in \overline{\mathcal{W}}$. Using the inequality (163), it can be shown that smooth elements of $\overline{\mathcal{W}}$ must lie in $\mathcal{W}$. Thus, for any $\gamma \in \mathcal{U} \cap \mathcal{V}$, we have $W_{\Sigma}\left(g ; \gamma^{\prime}, \gamma\right)=0$ for all $\gamma^{\prime} \in \mathcal{V}$ if and only if $\gamma \in \mathcal{W}$.

We now restrict consideration to axisymmetric perturbations, $\mathscr{L}_{\psi_{A}} \gamma=0$ (see (52)). We can redefine the spaces $\mathcal{K}, \mathcal{W}$, and $\mathcal{V}$ with the word "axisymmetric" suitably inserted, and all of the results of this subsection and their proofs continue to hold without modification. In order not to make our notation more cumbersome than necessary, we shall continue to use $\mathcal{K}, \mathcal{W}$, and $\mathcal{V}$ to denote the axisymmetric versions of these spaces. Thus, even when not stated explicitly, axisymmetry should be understood in all statements below.

We now view the canonical energy $\mathcal{E}$, defined in section 2 , as a quadratic form on the (axisymmetric) Hilbert space $\mathcal{V}$, with dense domain $\mathcal{T} \equiv \mathcal{U} \cap \mathcal{V}$. We are interested in finding all of the elements $\gamma \in \mathcal{T}$ on which $\mathcal{E}$ is degenerate, i.e., for which

$$
\mathcal{E}\left(\gamma^{\prime}, \gamma\right)=0 \quad \text { for all } \gamma^{\prime} \in \mathcal{T}
$$

Since $\mathcal{E}\left(\gamma^{\prime}, \gamma\right)=W_{\Sigma}\left(g ; \gamma^{\prime}, \mathscr{L}_{t} \gamma\right)$, it follows immediately from Remark 2 above that $\mathcal{E}$ is degenerate on $\gamma \in \mathcal{T}$ if and only if $\mathscr{L}_{t} \gamma \in \mathcal{W}$. But, in the presence of axisymmetry, this is precisely the condition that $\gamma$ is a perturbation towards a stationary black hole (see Definition 2.1 at the end of subsection 2.3). Let $\mathcal{T}^{\prime}$ denote the space of equivalence classes of elements of $\mathcal{T}$, where two elements are equivalent if and only if they differ by a perturbation towards a stationary black hole. We have proven the following: The canonical energy $\mathcal{E}$ is well defined, non-degenerate quadratic form on $\mathcal{T}^{\prime} \times \mathcal{T}^{\prime}$.

\subsection{Stability and instability arguments}

For axisymmetric perturbations of a stationary black hole, we have just shown that $\mathcal{E}$ is well defined as a quadratic form on the space $\mathcal{T}^{\prime}$ of smooth solutions satisfying our gauge 
conditions with $\delta M=\delta J_{A}=\delta P_{i}=0$ modulo perturbations towards a stationary black hole. Since $\mathcal{E}$ is non-degenerate on $\mathcal{T}^{\prime}$, only the following two cases can occur:

- Case (a): $\mathcal{E}$ is positive semi-definite on $\mathcal{T}$ and hence is positive definite on $\mathcal{T}^{\prime}$.

- Case (b): There exists $\gamma \in \mathcal{T}$ such that $\mathcal{E}(\gamma, \gamma)<0$.

We now analyze the behavior of perturbations in these two cases.

Case (a): $\mathcal{E}$ provides a positive definite conserved norm on $\mathcal{T}^{\prime}$. This precludes existence of "growing modes" in $\mathcal{T}^{\prime}$. But elements of $\mathcal{T}$ can be expressed as the sum of a representative element of $\mathcal{T}^{\prime}$ and a perturbation towards a stationary black hole. Since the latter perturbations are manifestly stable, this shows stability for perturbations in $\mathcal{T}$. However, by Proposition 5, $\mathcal{T}$ is dense in $\mathcal{V}$. Now, a general axisymmetric solution to the constraints in $\mathcal{K}$ can be written as a sum of an element of $\mathcal{V}$ and a finite linear combination of representative "change of mass," "change of angular momentum," and "change of linear momentum" perturbations. The desired "change of linear momentum" perturbations can be chosen to be $\mathscr{L}_{Y_{i}} g$ where $Y_{i}$ is an asymptotic boost. These are manifestly stable. If the black hole is part of a family parametrized 30 by $\left(M, J_{A}\right)$, then the "change of mass" and "change of angular momentum" perturbations can be chosen to be the perturbations towards other members of this family. These are also manifestly stable. Thus, we conclude that the black hole is stable to a dense set of axisymmetric perturbations in the subspace of $\mathcal{K}$ comprised by solutions to the constraints.

Furthermore, the results of section 3 strongly suggest that for perturbations $\gamma \in \mathcal{T}, \overline{\mathcal{E}}$ and $\mathcal{E}$ should decay to zero on the slices $\mathscr{S}(t)$ as $t \rightarrow \infty$, and thus, at late times, $\gamma$ should approach a perturbation towards a stationary black hole.

Case (b): Let $\gamma \in \mathcal{T}$ be such that $\mathcal{E}(\gamma, \gamma)<0$. We obtain a contradiction with the possibility that $\gamma$ approaches a perturbation, $\gamma_{0}$, towards a stationary black hole on $\mathscr{S}(t)$ as $t \rightarrow \infty$ as follows. If $\gamma$ approached a stationary solution, then the Bondi news and the shear of the horizon should approach zero at asymptotically late times, in which case as $t \rightarrow \infty$ we should have $\overline{\mathcal{E}}(\gamma, \mathscr{S}(t)) \rightarrow \mathcal{E}(\gamma, \mathscr{S}(t)) \rightarrow \mathcal{E}\left(\gamma_{0}\right)=0$. However, this is a contradiction because for all $t$ we have $\overline{\mathcal{E}}(\gamma, \mathscr{S}(t)) \leq \overline{\mathcal{E}}(\gamma, \Sigma)=\mathcal{E}(\gamma, \Sigma)<0$.

Although this contradiction does not prove instability, the fact that $|\overline{\mathcal{E}}(\gamma, \mathscr{S}(t))|$ can only increase with time suggests that the amplitude of the perturbation does not decrease with time. But then the fluxes through the horizon $\mathscr{H}^{+}$, and null-infinity $\mathscr{I}^{+}$, should also not decrease, causing $|\overline{\mathcal{E}}(\gamma, \mathscr{S}(t))|$ to increase further, etc. It therefore seems highly plausible that $\overline{\mathcal{E}}(\gamma, \mathscr{S}(t)) \rightarrow-\infty$ as $t \rightarrow \infty$ and that the amplitude of the perturbation grows without bound, as has been previously argued in a different context in [11, 12, 13, 14.

Thus, we conclude that in Case (a) the black hole is stable, whereas in Case (b), the black hole is unstable. In other words, we have argued that the necessary and sufficient condition for black hole stability is that the canonical energy $\mathcal{E}$ be positive semi-definite on the space $\mathcal{T}=\mathcal{U} \cap \mathcal{V}$ of smooth solutions with $\delta M=\delta J_{A}=\delta P_{i}=0$ that satisfy our horizon boundary conditions $\left.\delta \vartheta\right|_{B}=\left.\delta \epsilon\right|_{B}=0$.

\footnotetext{
${ }^{30}$ Even if the black hole is not part of a family parameterized by $\left(M, J_{A}\right)$, it should be possible to show existence of a basis of stable "change of mass" and "change of angular momentum" perturbations.
} 
We now briefly indicate some possible ways of improving the above arguments. To prove stability in Case (a), we would like to establish boundedness of $\gamma$ itself rather than have boundedness/conservation of a norm on $\gamma$. However, this is clearly not possible until we have imposed suitable gauge conditions on $\gamma$ that uniquely fix its evolution. Indeed, our present gauge conditions restrict $\gamma$ only near infinity and near $\mathscr{H}^{+}$, so they allow pure gauge perturbations for which $\gamma$ becomes arbitrarily large. If suitable gauge conditions are imposed on $\gamma$, then it is not implausible that $\mathcal{E}$ would provide a conserved norm on $\gamma \in \mathcal{T}$ that would be equivalent to a Sobolev norm. The canonical energy of the perturbation $\left(\mathscr{L}_{t}\right)^{k} \gamma$ would similarly provide a family of conserved norms on $\gamma \in \mathcal{T}$ that plausibly would be equivalent to higher Sobolev norms. If so, boundedness of $\gamma$ itself would be proven.

It does not seem feasible to try to directly convert the above argument for instability in Case (b) into a mathematically rigorous proof. However, it is possible that a proof of instability in Case (b) [as well as stability in Case (a)] could be obtained along the following lines. Again, to get started, it should be necessary to suitably fix a gauge so as to obtain deterministic dynamics. Consider, first, the case of perturbations off of a static black hole. On account of the time reflection symmetry of the background, $\mathcal{E}$ will be invariant under time reflections, so the integral expression for $\mathcal{E}$ in terms of initial data will not contain any "cross-terms" between $\delta p$ and $\delta h$. Thus, $\mathcal{E}$ can be written as a sum of a "kinetic energy" (quadratic in $\delta p$ ) and a "potential energy" (quadratic in $\delta h$ ). It is likely that the kinetic energy is always positive-definite. For the case of spherically symmetric perturbations (with matter fields) off of a static, spherically symmetric background, it was shown in [35] that the kinetic energy could be used to define an inner product such that the potential energy could be expressed as the expectation value of a self-adjoint operator $A$ that appears in the dynamical evolution equations. In this case, it can be seen directly from the dynamical evolution equations that positivity of $A$ is equivalent to stability. However, since the kinetic energy is positive, positivity of $A$ is equivalent to positivity of $\mathcal{E}$, thus rigorously establishing the equivalence of dynamical stability and positivity of $\mathcal{E}$. It is not inconceivable that similar results could be proven for perturbations of an arbitrary static black hole, although, obviously, a number of technical details would have to be sorted out. A similar strategy could also be applied to the stationary-axisymmetric case - making use of the $t-\psi_{A}$ reflection isometry to decompose initial data into its "time symmetric" and "time antisymmetric" parts - but it would be less obvious in this case that the "kinetic energy" would have to be positive.

\section{Proof of the Gubser-Mitra conjecture}

Up to this point, we have restricted our considerations to the stability of black holes. In this section, we extend our considerations to black branes of the form (1). By making the same type of dynamical instability argument as given in subsection 4.2 above, we will show that if a family of black holes is thermodynamically unstable, then the corresponding family of black branes must be dynamically unstable.

To begin, suppose we have a family of stationary, axisymmetric black holes, $g_{a b}\left(M, J_{A}\right)$, in $D \geq 4$ dimensions, that satisfy the asymptotic and gauge conditions of subsection 2.1. Let $\vec{\xi}=\left(M_{0}, J_{0 A}\right)$ denote the parameter values of a particular black hole in this family, 
and consider the one-parameter subfamily

$$
g_{a b}(\lambda):=g_{a b}(\vec{\xi}+\lambda \vec{v}),
$$

where $\vec{v}$ is an arbitrary vector in the parameter space $\left(M, J_{A}\right)$. It is obvious that for this family, we have

$$
\frac{d^{2} M}{d \lambda^{2}}(0)=\frac{d^{2} J_{A}}{d \lambda^{2}}(0)=0
$$

Thus, by (153), for the perturbation determined by $\vec{v}$ we have

$$
\mathcal{E}=-\frac{\kappa}{8 \pi} \frac{d^{2} A}{d \lambda^{2}}(0)
$$

On the other hand, we have

$$
\frac{d^{2} A}{d \lambda^{2}}(0)=\left.\operatorname{Hess}_{A}\right|_{\vec{\xi}}(\vec{v}, \vec{v})
$$

where $\left.\operatorname{Hess}_{A}\right|_{\vec{\xi}}$ denotes the Hessian of $A$ [see eq.(2)] at parameter value $\vec{\xi}$. Thus, we see that one can find a perturbation to a black hole in the family that makes $\mathcal{E}$ negative if and only if the Hessian $\operatorname{Hess}_{A}$ has a positive eigenvalue.

However, a nontrivial perturbation to a black hole in the family clearly does not have $\delta M=\delta J_{A}=0$, so a negative value of $\mathcal{E}$ - or, equivalently, a positive eigenvalue of Hess $A_{-}$ does not provide any information about stability. However, consider now the $(D+p)$ dimensional spacetime $\tilde{\mathscr{M}}=\mathscr{M} \times \mathbb{T}^{p}$ with metric $\tilde{g}_{a b}$ defined as above in eq. (1) with each $z_{i}$ a $2 \pi l$-periodic coordinate parameterizing the corresponding "extra-dimension". (Here and in the following, a tilda dentotes a quantity associated with the $(D+p)$-dimensional spacetime $\tilde{\mathscr{M}}$.) This metric represents a "uniform black brane", where uniform refers to the fact that each $\left(\partial / \partial z_{i}\right)^{a}$ is a Killing field of this spacetime, and where brane refers to the fact that the horizon cross section is now $\tilde{B}=B \times \mathbb{T}^{p}$. A black brane spacetime is "asymptotically Kaluza-Klein (KK)" rather than asymptotically flat, but the ADM conserved quantities $(\tilde{M}, \tilde{\mathbf{P}}, \tilde{\mathbf{J}}, \tilde{\mathbf{C}})$ can be defined as in the asymptotically flat case (see eq.(37)). In addition, we have conserved quantities ("KK charges"), $\tilde{\mathbf{T}}$, associated with the asymptotic symmetries $\left(\partial / \partial z_{i}\right)^{a}$, given by

$$
\delta \tilde{T}_{i}:=\int_{\infty}\left[\delta Q_{\partial / \partial z^{i}}(\tilde{g})-i_{\partial / \partial z^{i}} \theta(\tilde{g}, \delta \tilde{g})\right]
$$

We now prove the following proposition:

Proposition 6. Let $g_{a b}\left(M, J_{A}\right)$ be a family of black holes that is thermodynamically unstable at $\left(M_{0}, J_{0 A}\right)$, i.e., there exists a perturbation $\vec{v}$ within the black hole family for which $\mathcal{E}<0$, which will be the case if $\operatorname{Hess}_{A}$ has a positive eigenvalue at $\left(M_{0}, J_{0 A}\right)$. Then, for any black brane corresponding to $g_{a b}\left(M_{0}, J_{0 A}\right)$ via eq.(1) with sufficiently large $l$, one can find a perturbation for which $\tilde{\mathcal{E}}<0$ and $\delta \tilde{M}=\delta \tilde{J}_{A}=\delta \tilde{P}_{i}=\tilde{T}_{i}=0,\left.\delta \tilde{\vartheta}\right|_{\tilde{B}}=\left.\delta \tilde{\epsilon}\right|_{\tilde{B}}=0$.

Proof: For notational simplicity, we prove the proposition for $p=1$ extra dimension, the general case is completely analogous. Let $\left(\delta p^{a b}, \delta h_{a b}\right)$ denote the initial data of a perturbation $\vec{v}$ in the black hole family for which $\mathcal{E}<0$. Without loss of generality, 
we may assume that our Cauchy surface $\Sigma$ is chosen to be maximal in the background and perturbed spacetime 31 , so that $p^{a b} h_{a b}=0$, and $\delta p^{a b} h_{a b}+p^{a b} \delta h_{a b}=0$. We wish to construct initial data $\left(\delta \tilde{p}^{a b}, \delta \tilde{h}_{a b}\right)$ in the $(D+1)$-dimensional black brane spacetime with $\tilde{\mathcal{E}}<0$ for which $\delta \tilde{M}=\delta \tilde{J}_{A}=\delta \tilde{P}_{i}=\delta \tilde{T}=\delta \tilde{A}=0$ and for which $\delta \tilde{\vartheta}=0$ at $\tilde{B}$.

Our ansatz for $\left(\delta \tilde{p}^{a b}, \delta \tilde{h}_{a b}\right)$ is

$$
\begin{aligned}
\delta \tilde{p}^{a b}= & \left(\delta p^{a b}+\frac{1}{D-1} p^{a b} \phi+h^{\frac{1}{2}}\left\{D^{(a} X^{b)}+D^{(a} \zeta\left(\frac{\partial}{\partial z}\right)^{b)}-\frac{1}{D}\left(h^{a b}+\left(\frac{\partial}{\partial z}\right)^{a}\left(\frac{\partial}{\partial z}\right)^{b}\right) D^{c} X_{c}\right.\right. \\
& \left.\left.+i k / l\left(\frac{\partial}{\partial z}\right)^{(a} X^{b)}+\frac{D-1}{D} i k / l \zeta\left(\frac{\partial}{\partial z}\right)^{(a}\left(\frac{\partial}{\partial z}\right)^{b)}-\frac{1}{D} i k / l \zeta h^{a b}\right\}\right) \mathrm{e}^{i z \cdot k / l}, \\
\delta \tilde{h}_{a b}= & \left(\delta h_{a b}-\frac{1}{D-1} h_{a b} \phi\right) \mathrm{e}^{i z \cdot k / l}
\end{aligned}
$$

where $k \in \mathbb{Z}$ and $\phi, \zeta, X^{a}$ are tensor fields on $\Sigma$ (so they are independent of $z$, and tangent to $\Sigma$ ). The terms in $\delta \tilde{p}^{a b}$ involving $\zeta, X^{a}$ may be written alternatively as $\tilde{D}^{(a} \tilde{X}^{b)}-$ $\frac{1}{D} \tilde{h}^{a b} \tilde{D}^{c} \tilde{X}_{c}$, where

$$
\tilde{X}^{a}=\left(\zeta\left(\frac{\partial}{\partial z}\right)^{a}+X^{a}\right) \mathrm{e}^{i k \cdot z / l} .
$$

If $k \in \mathbb{Z}$ is not zero, then it is easily seen that the surface integrals defining $\delta \tilde{M}$, $\delta \tilde{J}_{A}, \delta \tilde{P}_{i}, \delta \tilde{T}$ and $\delta \tilde{A}$ vanish because the $z$-dependence is $\mathrm{e}^{i z \cdot k / l}$.

The tensor fields $\phi, \zeta, X^{a}$ in our ansatz (132) are now chosen so that the linearized momentum and Hamiltonian constraints are satisfied. It can be seen that the satisfaction of the linearized momentum constraint is equivalent to

$$
\tilde{D}_{a}\left(\tilde{D}^{(a} \tilde{X}^{b)}-\frac{1}{D} \tilde{h}^{a b} \tilde{D}^{c} \tilde{X}_{c}\right)=\frac{1}{2} i k / l h^{-\frac{1}{2}} \delta h_{c d} p^{c d} \mathrm{e}^{i k \cdot z / l}\left(\frac{\partial}{\partial z}\right)^{b} .
$$

This is an equation on $\tilde{\Sigma}$, but because all tensor fields have the same dependence $\mathrm{e}^{i k \cdot z / l}$ on $z$, we can rewrite it as the following system of equations on $\Sigma$ :

$$
\begin{aligned}
& D_{a}\left(D^{(a} X^{b)}-\frac{1}{D} h^{a b} D^{c} X_{c}\right)-\frac{1}{2} k^{2} / l^{2} X^{b}+\frac{D-2}{2 D} i k / l D^{b} \zeta=0 \\
& D^{a} D_{a} \zeta-2 \frac{D+1}{D} k^{2} / l^{2} \zeta+\frac{D-2}{D} i k / l D^{a} X_{a}=i k / l h^{-\frac{1}{2}} \delta h_{c d} p^{c d} .
\end{aligned}
$$

The satisfaction of the Hamiltonian constraint is equivalent to the linearized Lichnerowicz equation for $\phi$,

$$
\begin{aligned}
& \left(-D^{a} D_{a}+h^{-1} p_{a b} p^{a b}+k^{2} / l^{2} \frac{(D-1)}{(D-2)}\right) \phi= \\
& =\frac{(D-1)}{(D-2)} k^{2} / l^{2} \delta h_{a}{ }^{a}-2 \frac{(D-1)}{(D-2)} p^{a b} D_{a} X_{b} h^{-\frac{1}{2}} .
\end{aligned}
$$

Together, the eqs. (136), (135) form a system of linear, inhomogeneous, elliptid 32 PDE's for the unknown tensor fields $\phi, \zeta, X^{a}$ on $\Sigma$. We need to impose boundary conditions

\footnotetext{
${ }^{31}$ Existence of a maximal slice in the background spacetime is shown in 3 . For the perturbed spacetime, existence of a slice that preserves maximality follows from the fact that the perturbed lapse required to achieve this condition satisfies a suitable elliptic equation.

${ }^{32}$ Indeed, the principal symbol of the equation for $X^{a}$ in (135) is $\sigma(\xi)^{a}{ }_{b}=h^{c d} \xi_{d} \xi_{c} \delta^{a}{ }_{b}-(1 / D) h^{a c} \xi_{c} \xi_{b}$, which is an invertible linear map in each tangent space $T \Sigma$ for any $T^{*} \Sigma \ni \xi_{a} \neq 0$.
} 
at $B$. To find these, we consider the linearized expansion $\delta \tilde{\vartheta}$ on $\tilde{B}$ in the black brane spacetime. We would like this to vanish. It is calculated using eq. (116) as

$$
\begin{aligned}
\delta \tilde{\vartheta}= & \left(-\frac{3}{2} \phi h^{-\frac{1}{2}} p_{a b}\left(h^{a b}-\eta^{a} \eta^{b}\right)-\frac{D-2}{2} \eta^{a} D_{a} \phi+\frac{1}{2} \phi \mathscr{L}_{\eta} h_{a b}\left(h^{a b}-\eta^{a} \eta^{b}\right)\right. \\
& \left.-\eta^{a} \eta^{b} D_{a} X_{b}+\frac{1}{D} D^{c} X_{c}+\frac{1}{D} i k / l \zeta\right) \mathrm{e}^{i k \cdot z / l} \\
= & \left(-\frac{D-2}{2} \eta^{a} D_{a} \phi+2 H \phi-\eta^{a} \eta^{b} D_{a} X_{b}+\frac{1}{D} D^{c} X_{c}+\frac{1}{D} i k / l \zeta\right) \mathrm{e}^{i k \cdot z / l} \\
= & \left(-\frac{D-2}{2} \mathscr{L}_{\eta} \phi+2 H \phi-\frac{D-1}{D} \mathscr{L}_{\eta}\left(\eta^{a} X_{a}\right)+\right. \\
& \left.\frac{1}{D} H\left(\eta^{a} X_{a}\right)+\frac{1}{D} \mathcal{D}_{a}\left[\left(h^{a b}-\eta^{a} \eta^{b}\right) X_{b}\right]+\frac{1}{D} i k / l \zeta\right) \mathrm{e}^{i k \cdot z / l} \text { at } \tilde{B},
\end{aligned}
$$

where $\eta^{a}$ is the normal to $B$ within $\Sigma, \mathcal{D}_{a}$ is the derivative operator intrinsic to $B$, and $H$ is the trace of the extrinsic curvature of $B$ within $\Sigma$. In the first line, use has been made of the fact that $p_{a}{ }^{a}=0$ and $\delta \vartheta=0$ on $B$ in the original spacetime. In the second line, we used that $0=\vartheta=\left(h^{a b}-\eta^{a} \eta^{b}\right)\left(h^{-\frac{1}{2}} p_{a b}+\frac{1}{2} \mathscr{L}_{\eta} h_{a b}\right)$ on $B$ in the original spacetime. In the last step we have, without loss of generality, extended $\eta^{a}$ of $B$ such that it is geodesic. Because $B$ is the bifurcation surface of a Killing horizon, $H=0$ on $B$. In view of this, $\delta \tilde{\vartheta}=0$ on $\tilde{B}$ will follow if we impose on $\phi, \zeta, X^{a}$ the boundary conditions

$$
\begin{aligned}
\left.\mathscr{L}_{\eta} \phi\right|_{B} & =0, \\
\left.\left(h^{a b}-\eta^{a} \eta^{b}\right) X_{b}\right|_{B} & =0, \\
\left.\mathscr{L}_{\eta}\left(X^{b} \eta_{b}\right)\right|_{B} & =0, \\
\left.\zeta\right|_{B} & =0 .
\end{aligned}
$$

In other words, we impose Neumann conditions on $\phi$, Dirichlet conditions on $\zeta$, Neumann conditions on the orthogonal components $\eta^{c} X_{c}$ of $X^{a}$, and Dirichlet conditions on the tangent components $\left(h^{a b}-\eta^{a} \eta^{b}\right) X_{b}$ of $X^{a}$ at $B$. We need to analyze the existence and properties of the solutions to (136), (135). The existence of a solution $\left(\zeta, X^{a}\right) \in \rho W_{\rho}^{1}(\Sigma)$ (and in fact $\in \rho W_{\rho}^{k}$ for any $k$ ) follows from lemma 4 in appendix B, because (135) is equivalent to (134), and the lemma gives a smooth solution $\tilde{X}^{a} \in \rho W_{\rho}^{1}(\tilde{\Sigma})$ to the latter equation. Next, we analyze the existence and properties of the solutions to (136). We need to distinguish the cases $D=4,5$ and $D \geq 6$.

$D \geq 6$ : In this case, the right side of $(136)$ is in $L^{2}$. Existence of a smooth solution $\phi$ in the Sobolev space $\rho W_{\rho}^{1}$ (and in fact $\in \rho W_{\rho}^{k}$ any $k$ ) satisfying Neumann boundary conditions can be proven as usual by considering the weak formulation of the boundary value problem

$$
\begin{aligned}
& \int_{\Sigma}\left(D^{a} \phi D_{a} \psi+h^{-1} p^{a b} p_{a b} \phi \psi+\frac{(D-1)}{(D-2)} k^{2} / l^{2} \phi \psi\right) h^{\frac{1}{2}} \\
= & \frac{(D-1)}{(D-2)} \int_{\Sigma}\left(k^{2} / l^{2} h^{\frac{1}{2}} \delta h_{a}{ }^{a} \psi-2 p^{a b} D_{a} X_{b} \psi\right)
\end{aligned}
$$

for all smooth $\psi$ with compact support in $\bar{\Sigma}$, and making use of the Poincare-type inequality

$$
c \int_{\Sigma} \rho^{-2} \psi^{2} h^{\frac{1}{2}} \leq \int_{\Sigma} D^{a} \psi D_{a} \psi h^{\frac{1}{2}}
$$


for some $c>0$. Since these arguments are standard and very similar to those in the proof of lemma 3 in appendix $\mathrm{B}$, we do not repeat them here.

It remains to be shown that the normalized canonical energy $\tilde{\mathcal{E}}=1 /(2 \pi l) \tilde{W}_{\tilde{\Sigma}}\left(\delta \tilde{g}, \mathscr{L}_{t} \delta \tilde{g}\right)$ on the $(D+1)$-dimensional black brane spacetime approaches the canonical energy $\mathcal{E}<0$ of the original black hole perturbation for $l \rightarrow \infty$. For this, it is important to understand how the norms of $\phi_{l}, \zeta_{l}, X_{l}{ }^{a}$ behave for large $l$ (here we put an "l" on the tensor fields to indicate their dependence on $l$ ). We use that the source term in (134) is of order $O\left(\rho^{D-1}\right)$, so $\rho$ times the source is in $W_{\rho}^{1}(\tilde{\Sigma})$ in $D \geq 6$ and tends to zero in that norm 33 . From lemma 4 of appendix B we infer that the $L^{2}(\tilde{\Sigma})$-norms of $\rho^{-1} \tilde{X}_{l}^{a}, \rho^{-1} \tilde{D}_{b} \tilde{X}_{l}^{a}, \rho \tilde{D}_{a} \tilde{D}_{b} \tilde{X}_{l}^{c}$ tend to zero of order $O(1 / l)$ as $l \rightarrow \infty$. This translates into the statement that the $L^{2}(\Sigma)$ norms of $\rho^{-1} \zeta_{l}, \rho^{-1} X_{l}^{a}, D_{b} X_{l}^{a}, D_{a} \zeta_{l}, \rho / l D_{a} \zeta_{l}, \rho / l D_{a} X_{l}^{b}, 1 / l \zeta_{l}, 1 / l X_{l}^{a}$ as well as $\rho D_{a} D_{b} \zeta_{l}, \rho D_{a} D_{b} X_{l}^{c}$ tend to zero of order $O(1 / l)$ as $l \rightarrow \infty$. It follows immediately from (136) that the $L^{2}$-norm of $1 / l \phi_{l}$ remains bounded as $l \rightarrow \infty$. Then, combining (139) with (140) for $\phi=\psi=\phi_{l}$, we find that $\rho^{-1} \phi_{l}, D_{a} \phi_{l} \rightarrow 0$ in the sense of $L^{2}$ as $l \rightarrow \infty$. By standard bootstrap and scaling arguments for elliptic PDE's of the nature under consideration (see e.g. 6] Appendix A for a discussion and references), it follows similarly $\rho D_{a} D_{b} \phi_{l} \rightarrow 0$ in $L^{2}$.

Using now the conditions $\delta p^{a b} h_{a b}+p^{a b} \delta h_{a b}=0, p^{c}{ }_{c}=0$ (from which follow the same for the corresponding tilde tensor fields on $\tilde{\Sigma}$ ), the boundary conditions at $B$, and eliminating

\footnotetext{
${ }^{33}$ In $D=4,5$ this is still true if we impose a gauge, as below, that $\delta h_{a b}-\frac{1}{D-1} h_{a b} \delta h$ is of order $O\left(\rho^{-(D-2)}\right)$, because $p_{a}{ }^{a}=0$.
} 
terms that are total derivatives with respect to $z$, we obtain 34 from (866)

$16 \pi \tilde{\mathcal{E}}-16 \pi \mathcal{E}=$

$\operatorname{Re} \int_{\Sigma} N\left\{\frac{1}{2} R_{a b}(h) \delta_{1} h_{c}{ }^{c *} \delta_{2} h^{a b}+\frac{1}{2} R_{a b}(h) \delta_{1} h^{a b *} \delta_{2} h_{c}{ }^{c}-2 R_{a c}(h) \delta_{1} h^{a b *} \delta_{2} h_{b}^{c}-\right.$

$\frac{1}{4} \delta_{1} h^{a c *} D_{a} D_{c} \delta_{2} h_{b}{ }^{b}-\frac{1}{4} D_{a} D_{c} \delta_{1} h_{b}^{b *} \delta_{2} h^{a c}-\frac{1}{4} \delta_{1} h^{a c *} D^{b} D_{b} \delta_{2} h_{a c}-\frac{1}{4} \delta_{2} h^{a c *} D^{b} D_{b} \delta_{1} h_{a c}+$

$\frac{1}{2} \delta_{1} h^{a c *} D_{b} D_{a} \delta_{2} h_{c}{ }^{b}+\frac{1}{2} D_{b} D_{a} \delta_{1} h_{c}{ }^{b *} \delta_{2} h^{a c}-\frac{3}{4} D_{a} D^{a}\left(\delta_{1} h^{b c *} \delta_{2} h_{b c}\right)-$

$\frac{3}{4} D_{a}\left(\delta_{1} h^{a b *} D_{b} \delta_{2} h_{c}{ }^{c}+\delta_{2} h^{a b} D_{b} \delta_{1} h_{c}{ }^{c *}\right)+\frac{1}{4} D^{a} D_{a}\left(\delta_{1} h_{b}{ }^{b *} \delta_{2} h_{c}{ }^{c}\right)+$

$D_{a}\left(\delta_{1} h_{c}{ }^{a *} D_{b} \delta_{2} h^{c b}+\delta_{2} h^{a}{ }_{c} D_{b} \delta_{1} h^{c b *}\right)+\frac{1}{2} D_{a}\left(\delta_{1} h_{c}{ }^{b *} D_{b} \delta_{2} h^{a c}+\delta_{2} h_{c}{ }^{b} D_{b} \delta_{1} h^{a c *}\right)-$

$\left.\frac{1}{4} D^{a}\left(\delta_{1} h_{d}{ }^{d *} D^{b} \delta_{2} h_{a b}+\delta_{2} h_{d}^{d} D^{b} \delta_{1} h_{a b}{ }^{*}\right)\right\} h^{\frac{1}{2}}+$

$\operatorname{Re} \int_{\Sigma} N\left\{\frac{1}{2} p^{a b} p_{a b} \delta_{1} h_{a}^{a *} \delta_{2} h_{b}{ }^{b}-\frac{3}{2} p^{c}{ }_{b} p^{a b}\left(\delta_{1} h_{d}{ }^{*} \delta_{2} h_{a c}+\delta_{2} h_{d}{ }^{d} \delta_{1} h_{a c}{ }^{*}\right)+\right.$

$2 \delta_{1} p^{a b *} \delta_{2} p_{a b}+2 p^{a c} p^{b d} \delta_{1} h_{a b}{ }^{*} \delta_{2} h_{c d}-\frac{3}{2} \delta_{1} h_{d}^{d *} \delta_{2} p^{a b} p_{a b}-$

$\left.\frac{3}{2} \delta_{2} h_{d}{ }^{d} \delta_{1} p^{a b *} p_{a b}+4 p_{b}^{c}\left(\delta_{1} h_{a c}{ }^{*} \delta_{2} p^{a b}+\delta_{2} h_{a c} \delta_{1} p^{a b *}\right)\right\} h^{-\frac{1}{2}}-$

$\operatorname{Re} \int_{\Sigma} N^{a}\left\{-\delta_{1} p^{b c *} D_{a} \delta_{2} h_{b c}-\delta_{2} p^{b c} D_{a} \delta_{1} h_{b c}{ }^{*}+2 \delta_{1} p^{c b *} D_{b} \delta_{2} h_{a c}+\right.$

$2 \delta_{2} p^{c b} D_{b} \delta_{1} h_{a c}{ }^{*}+\delta_{1} h_{a c}{ }^{*} D_{b} \delta_{2} p^{b c}+\delta_{2} h_{a c} D_{b} \delta_{1} p^{c b *}-$

$\left.p^{c b}\left(\delta_{1} h_{a c}{ }^{*} D_{b} \delta_{2} h_{c}{ }^{d}+\delta_{2} h_{a d} D_{b} \delta_{1} h_{c}{ }^{* *}\right)+\frac{1}{2} p^{c b}\left(\delta_{1} h_{a d}{ }^{*} D^{d} \delta_{2} h_{c b}+\delta_{2} h_{a d} D_{b} \delta_{1} h_{c}{ }^{d *}\right)\right\}-$

$\operatorname{Re} \int_{B} \mu^{\frac{1}{2}} \frac{D-2}{D-1}\left(2 \delta h^{a b}-\frac{1}{D-1} \phi h^{a b}\right)^{*} \phi\left(h_{a b}-\eta_{a} \eta_{b}\right)+$

$\operatorname{Re} \int_{\Sigma} N\left\{\left(D_{a} \zeta\right)^{*} D^{a} \zeta+\frac{2}{D^{2}}\left(D_{a} X^{a}\right)^{*} D_{b} X^{b}+\frac{2(D-1)^{2}}{D^{2}} k^{2} / l^{2} \zeta^{*} \zeta+k^{2} / l^{2} X_{a}^{*} X^{a}+\right.$

$\left.\frac{1}{2} k^{2} / l^{2}\left(\delta h_{a b}-\frac{1}{D-1} \phi h_{a b}\right)^{*}\left(\delta h^{a b}-\frac{1}{D-1} \phi h^{a b}\right)\right\} h^{\frac{1}{2}}+$

$\operatorname{Re} \int_{\Sigma} N^{a}\left\{i k / l\left(\delta h_{a c}-\frac{1}{D-1} \phi h_{a c}\right)\left(D^{c} \zeta+i k / l X^{c}\right)^{*}\right\} h^{\frac{1}{2}}$.

\footnotetext{
${ }^{34}$ Note that $\mathcal{E}$ below is extended to complex-valued perturbations in such a way that it is anti-linear in the first argument; of course we could equally work with real valued perturbations by taking the real and imaginary parts of eqs. (132).
} 
where, in this equation, $*$ denotes complex conjugation. Here we have defined

$$
\begin{aligned}
\delta_{1} h_{a b} & =-\frac{1}{D-1} \phi h_{a b} \\
\delta_{2} h_{a b} & =2 \delta h_{a b}-\frac{1}{D-1} \phi h_{a b} \\
\delta_{1} p^{a b} & =\frac{1}{D-1} p^{a b} \phi+h^{\frac{1}{2}}\left\{D^{(a} X^{b)}-\frac{1}{D}\left(i k / l \zeta+D^{c} X_{c}\right)\right\} \\
\delta_{2} p^{a b} & =2 \delta p^{a b}+\frac{1}{D-1} p^{a b} \phi+h^{\frac{1}{2}}\left\{D^{(a} X^{b)}-\frac{1}{D}\left(i k / l \zeta+D^{c} X_{c}\right)\right\} .
\end{aligned}
$$

From this expression, together with the decay of the norms of the tensor fields $\zeta_{l}, \phi_{l}, X_{l}^{a}$ which we have described, it follow 35 that the terms on the right side of (141) go to zero as $l \rightarrow \infty$. Thus, we have $\tilde{\mathcal{E}} \rightarrow \mathcal{E}<0$ as $l \rightarrow \infty$, as we desired to show.

$D=4,5$ : In this case, the right side of (136) need not be in $L^{2}$, and the previous argument for obtaining a solution $\phi$ does not work directly. But in this case, we can apply the following modification to the above argument. We now write $\phi=\chi \delta h_{a}{ }^{a}+v$, where $\chi$ is equal to 1 near infinity, and equal to 0 in an open neighborhood of $B$. Imposing (136) on $\phi$ gives an equation for $v$ of the same type, but with a source in $L^{2}$ (and with the boundary condition $\mathscr{L}_{\eta} v=0$ at $B$ ). Existence of a smooth solution $v \in W^{1}$ again follows, and therefore existence of a solution $\phi$ follows, too. Now let $l \rightarrow \infty$ and denote the corresponding sequence of solutions by $v_{l}$. From the equation satisfied by $v_{l}$ it is easy to see that $D_{a} v_{l}$ is Cauchy in $L^{2}$, and, by (140), it follows that $\rho^{-1} v_{l}$ also is Cauchy. Hence $\rho^{-1} v_{l}, D_{a} v_{l}$ are convergent sequences in $L^{2}$, with limit $\rho^{-1} v \in W^{1}$. But it is easy to see that $v$ must be equal to $-\chi \delta h_{a}{ }^{a}$ since they both satisfy the same boundary value problem, so $\phi_{l}$ again satisfies $\rho^{-1} \phi_{l}, D_{a} \phi_{l}, \rho D_{a} D_{b} \phi_{l} \rightarrow 0$ in $L^{2}$. It then follows that all terms (141) again go to zero. The only terms for which the argument is somewhat different than for the case of $D \geq 6$ are the last two involving $\delta h_{a b}-\frac{1}{D-1} \phi_{l} h_{a b}$, because $\delta h_{a b}$ is not in $L^{2}$ now. For that term, we write $\delta h_{a b}-\frac{1}{D-1} \phi_{l} h_{a b}$ as $\left(\delta h_{a b}-\frac{1}{D-1} \delta h_{c}{ }^{c} h_{a b}\right)+\frac{1}{D-1}\left(\delta h_{c}{ }^{c}-\phi_{l}\right) h_{a b}$. Although $\delta h_{a b}$ is not in $L^{2}$ in dimensions $D=4,5$, we may assume that we are in a gauge 36 such that $\delta h_{a b}-\frac{1}{D-1} \delta h_{c}{ }^{c} h_{a b}$ falls off faster by one power of $\rho^{-1}$, implying that it is in $L^{2}$. Furthermore, we have $\left(-D^{a} D_{a}+h^{-1} p_{a b} p^{a b}\right) \phi_{l}=\frac{(D-1)}{(D-2)}\left[k^{2} / l^{2}\left(\phi_{l}-\delta h_{a}{ }^{a}\right)-2 p_{a b} D^{b} X_{l}^{a}\right]$. Because $\rho^{-1} \phi_{l}, D_{a} \phi_{l}, D_{b} X_{l}^{a} \rightarrow 0$ in $L^{2}$, it then follows (multiply by $\left(\phi_{l}-\delta h_{a}{ }^{a}\right)^{*}$ and integrate by parts) that $l^{-1}\left(\phi_{l}-\delta h_{a}{ }^{a}\right) \rightarrow 0$ in $L^{2}$. Thus, again $\tilde{\mathcal{E}} \rightarrow \mathcal{E}<0$.

Since $\delta \tilde{A}=0$, we can further change the initial data by a gauge transformation [i.e. adding $\sigma \tilde{\mathcal{L}}^{*}(\tilde{Y})$ with a suitable vector field $\tilde{Y}^{a}$ tangent to $\tilde{B}$, see eq. (77)] so that $\left.\delta \tilde{\epsilon}\right|_{\tilde{B}}=0$. For example, take $\tilde{Y}^{a}=\frac{1}{D-1}(i k / l)^{-1} \phi\left(\frac{\partial}{\partial z}\right)^{a} \mathrm{e}^{i k \cdot z / l}$ on $\tilde{\Sigma}$ near $\tilde{B}$, and vanishing in a neighborhood of infinity, and extend it off $\tilde{\Sigma}$ such that $\mathscr{L}_{t} \tilde{Y}^{a}=0$. By proposition 3 , this will not change $\tilde{\mathcal{E}}$.

\footnotetext{
${ }^{35}$ It is essential here to use the conditions $p_{a b}=O\left(\rho^{-(D-2)}\right), R_{a b}(h)=O\left(\rho^{-(D-1)}\right)$ as well as $\delta h_{a b}=$ $O\left(\rho^{-(D-3)}\right), \delta p^{a b}=O\left(\rho^{-(D-2)}\right)$. One can see that $\phi_{l}, \zeta_{l}, X_{l}^{a}$ and their derivatives only enter in the combinations such that their norm tends to zero in $L^{2}$, or such that the additional $1 / l$-factors give convergence to 0 .

${ }^{36}$ For example, for perturbations from a Schwarzschild to another Schwarzschild black hole, putting the metric perturbation into "isotropic coordinates" near infinity would provide such a gauge, and similarly for perturbations within the Kerr family.
} 
Essentially all of the analysis of sections 2, 3, and 4 can be applied straightforwardly to black branes. One important modification, however, is that in the definition of "perturbations toward stationary black branes," allowance must be made for the possibility that the horizon Killing field $K^{a}(\lambda)$ may correspond to a linear combination of the Killing fields $\left(\partial / \partial z^{i}\right)^{a}$ in addition to $t^{a}$ and $\psi_{A}^{a}$. Thus, definition 2.1 must be modified by including linear combinations of the Killing fields $\left(\partial / \partial z^{i}\right)^{a}$ in eq.(64). Correspondingly, in Proposition 4 , the requirement that $\gamma_{1}$ also satisfy $\delta T_{i}=0$ must be added to the hypothesis. In section 4, the definition of $\mathcal{W}$, eq.(110), (and, hence, $\mathcal{V}$ ) must be similarly modified to allow $X^{a}$ to coincide near infinity with a combination of the Killing fields $\left(\partial / \partial z^{i}\right)^{a}$ as well as rotational Killing fields and spatial translations. Correspondingly, in statement (2) of Proposition 5, the condition $\delta T_{i}=0$ must be added to the conditions $\delta M=\delta J_{A}=\delta P_{i}=0$. Aside from these changes, the results of the previous sections apply.

We have just shown that for a black hole family such that the Hessian matrix Hess $_{A}$ has a positive eigenvalue, there exists a perturbation of the corresponding black brane that satisfies $\delta \tilde{M}=\delta \tilde{J}_{A}=\delta \tilde{P}_{j}=\delta \tilde{T}_{i}=0$ together with $\left.\delta \tilde{\vartheta}\right|_{\tilde{B}}=\left.\delta \tilde{\epsilon}\right|_{\tilde{B}}=0$, and, for sufficiently large $l$, has $\tilde{\mathcal{E}}<0$. Since the flux analysis of section 3 for asymptotically flat spacetimes carries over to the asymptotically Kaluza-Klein case, we may make the same arguments as given for Case (b) of section 4.2 to conclude that the black brane must be unstable. Indeed for $D \geq 6$ the perturbation we have constructed lies in the black brane analog of $\mathcal{V}$, so we may make precisely the same arguments for instability. However, these arguments can also be made for the case $D=4,5$, since the black brane analog of Proposition 4 of section 2.3 yields a contradiction with the perturbation approaching a perturbation towards a stationary black brane at late times. Thus, we conclude that for any family of black holes that are thermodynamically unstable, the corresponding family of black branes is dynamically unstable to sufficiently long wavelength perturbations.

\section{Equivalence to the local Penrose inequality}

In this section, we show that for black holes, the satisfaction of the local Penrose inequality is equivalent to the positivity of canonical energy for perturbations with $\delta M=\delta J_{A}=$ $\delta P_{i}=0$ and hence, by our previous arguments, is equivalent to dynamical stability.

Let $\bar{g}_{a b}\left(M, J_{A}\right)$ be a family of stationary, axisymmetric, and asymptotically flat black hole metrics on $M$. Let $g_{a b}(\lambda)$ be a one-parameter family of axisymmetric metrics such that $g_{a b}(0)=\bar{g}_{a b}\left(M_{0}, J_{0 A}\right)$. Without loss of generality, we may assume (by applying an asymptotic Lorentz boost, if necessary) that the linear momentum of $g_{a b}(\lambda)$ vanishes, $P_{i}(\lambda)=0$. Let $M(\lambda), J_{A}(\lambda)$ denote the mass and angular momenta of $g_{a b}(\lambda)$. Let $\bar{g}_{a b}(\lambda)=$ $\bar{g}_{a b}\left(M(\lambda), J_{A}(\lambda)\right)$ denote the one-parameter family of stationary black holes with the same mass and angular momenta as $g_{a b}(\lambda)$. Let $\mathcal{A}(\lambda)$ denote the area of the apparent horizon of $g_{a b}(\lambda)$ on an initial slice and let $\overline{\mathcal{A}}(\lambda)$ denote the area of the event horizon of $\bar{g}_{a b}(\lambda)$. Then the following proposition holds:

Proposition 7. There exists a one-parameter family $g_{a b}(\lambda)$ for which

$$
\mathcal{A}(\lambda)>\overline{\mathcal{A}}(\lambda)
$$


to second order in $\lambda$ if and only if there exists a perturbation $\gamma_{a b}^{\prime}$ of $\bar{g}_{a b}\left(M_{0}, J_{0 A}\right)$ with $\delta M=\delta J_{A}=\delta P_{i}=0$ such that $\mathcal{E}\left(\gamma^{\prime}\right)<0$.

Proof: On account of our gauge condition $\left.\delta \vartheta\right|_{B}=0$, the apparent horizon of $g_{a b}(\lambda)$ coincides with $B$ to first order in $\lambda$. To find the apparent horizon of $g_{a b}(\lambda)$ to second order in $\lambda$, we would, in general, need to displace $B$ by a second order gauge transformation similar to (8) . However, since the extrinsic curvature of $B$ vanishes for the background metric $g_{a b}(0)$, the area will be unchanged to second order under such a displacement. Consequently, to second order in $\lambda$, we have $\mathcal{A}(\lambda)=A(\lambda)$, where $A(\lambda)$ denotes the area of $B$ in the metric $g_{a b}(\lambda)$. Similarly, to second order in $\lambda$, we have $\overline{\mathcal{A}}(\lambda)=\bar{A}(\lambda)$.

Obviously, we have $A(0)=\bar{A}(0)$, as the two families coincide for $\lambda=0$. Likewise, by the first law of black hole mechanics, we have

$$
\frac{d A}{d \lambda}(0)=\frac{8 \pi}{\kappa}\left[\frac{d M}{d \lambda}(0)-\sum \Omega_{A} \frac{d J_{A}}{d \lambda}(0)\right]=\frac{d \bar{A}}{d \lambda}(0)
$$

Thus, what matters is the second derivative of the areas. Let $\gamma_{a b}=\left.\left(d g_{a b} / d \lambda\right)\right|_{\lambda=0}$ and let $\bar{\gamma}_{a b}=\left.\left(d \bar{g}_{a b} / d \lambda\right)\right|_{\lambda=0}$. Since the families have the same mass and angular momenta, our second variation formula (53) gives

$$
\begin{aligned}
\frac{\kappa}{8 \pi}\left[\frac{d^{2} A}{d \lambda^{2}}(0)-\frac{d^{2} \bar{A}}{d \lambda^{2}}(0)\right] & =\mathcal{E}(\bar{\gamma}, \bar{\gamma})-\mathcal{E}(\gamma, \gamma) \\
& =-\mathcal{E}\left(\gamma^{\prime}, \gamma^{\prime}\right)+2 \mathcal{E}\left(\gamma^{\prime}, \bar{\gamma}\right)
\end{aligned}
$$

where we have defined $\gamma_{a b}^{\prime}=\bar{\gamma}_{a b}-\gamma_{a b}$. However, $\gamma_{a b}^{\prime}$ is clearly a linearized perturbation with $\delta M=\delta J_{A}=\delta P_{i}=0$, and $\bar{\gamma}_{a b}$ is manifestly a perturbation towards a stationary black hole. Therefore, the second term vanishes by Proposition 4 of section 2.3. Thus, we have

$$
\frac{\kappa}{8 \pi}\left[\frac{d^{2} A}{d \lambda^{2}}(0)-\frac{d^{2} \bar{A}}{d \lambda^{2}}(0)\right]=-\mathcal{E}\left(\gamma^{\prime}, \gamma^{\prime}\right)
$$

from which the proposition follows immediately.

As explained in the introduction, the satisfaction of the inequality (143) is incompatible with the stability of the family of black holes $\bar{g}_{a b}\left(M, J_{A}\right)$. It is therefore reassuring that we have found that satisfaction of this inequality is equivalent to the condition that we have obtained in this paper for dynamical instability.

Acknowledgments: The impetus for this work arose from a talk given by Harvey Reall at the workshop "Numerical Relativity Beyond Astrophysics" (Edinburgh, July, 2011) on the research in [10] and discussions by one of us (R.M.W.) with Reall following that talk. We would also like to thank P. Chrusciel and E. Delay for explanations about gluing constructions. S.H. would like to thank M. Marletta for discussions about boundary value problems. The research of R.M.W. was supported in part by NSF grants PHY 08-54807 and PHY 12-02718 to the University of Chicago. S.H. acknowledges financial support through ERC grant QC \& C 259562 . 


\section{A Proof of Lemma 2}

Lemma 2. Let $\delta g_{a b}$ be a solution to the linearized Einstein equations satisfying our asymptotic flatness conditions and our gauge conditions (7) and (11) at B. Suppose in addition that $\delta A=0$ (so that, by (11), we have $\left.\delta \epsilon\right|_{B}=0$ ) and that $\delta H_{X}=0$ for some asymptotic symmetry $X^{a}$. Then $W_{\Sigma}\left(g ; \delta g, \mathscr{L}_{\xi} g\right)=0$ for all smooth $\xi^{a}$ such that (i) $\left.\xi^{a}\right|_{B}$ is tangent to the generators of $\mathscr{H}^{+}$and (ii) $\xi^{a}$ approaches a multiple of $X^{a}$ as $\rho \rightarrow \infty$. Conversely, if $\delta g_{a b}$ is smooth and asymptotically flat and if $W_{\Sigma}\left(g ; \delta g, \mathscr{L}_{\xi} g\right)=0$ for all such $\xi^{a}$, then $\delta g_{a b}$ is a solution to the linearized Einstein equation with $\left.\delta \vartheta\right|_{B}=\left.\delta \epsilon\right|_{B}=0$ at $B$ and with $\delta H_{X}=0$.

Proof: For a solution $\delta g_{a b}$, our fundamental variation formula (36) yields

$$
\begin{aligned}
W_{\Sigma}\left(g ; \delta g, \mathscr{L}_{\xi} g\right) & =\int_{\infty}\left[\delta Q_{\xi}(g)-i_{\xi} \theta(g ; \delta g)\right]-\int_{B}\left[\delta Q_{\xi}(g)-i_{\xi} \theta(g ; \delta g)\right] \\
& =-\int_{B}\left[\delta Q_{\xi}(g)-i_{\xi} \theta(g ; \delta g)\right]+\delta H_{\xi} .
\end{aligned}
$$

Since $\xi^{a} \rightarrow c X^{a}$ for some constant $c$, we have $\delta H_{\xi}=c \delta H_{X}$.

We now evaluate the boundary term from $B$. To do so, we write $n^{a}=(\partial / \partial u)^{a}, l^{a}=$ $(\partial / \partial r)^{a}$, where $(u, r)$ are the Gaussian normal coordinates of (5). The condition that $\left.\xi^{a}\right|_{B}$ is tangent to the generators of $\mathscr{H}^{+}$implies that we can decompose it as

$$
\xi^{a}=f n^{a}+u Z^{a}+r Y^{a}
$$

where $Y^{a}, Z^{a}$ are smooth but otherwise arbitrary. In Gaussian null coordinates (5), the metric perturbation takes the form

$$
\delta g_{a b}=-2 \nabla_{(a} u\left(r^{2} \delta \alpha \nabla_{b)} u+r \delta \beta_{b)}\right)+\delta \mu_{a b}
$$

where $n^{a} \delta \beta_{a}=l^{a} \delta \beta_{a}=n^{a} \delta \mu_{a b}=l^{a} \delta \mu_{a b}=0$.

We now calculate $\left.i_{\xi} \theta(g ; \delta g)\right|_{B}$ [see eq. (17)]. For the pull-back to $B$, we have

$$
\left(i_{\xi} \theta\right)_{a_{1} \ldots a_{D-2}}=\frac{1}{16 \pi} f v^{c} n_{c} \epsilon_{a_{1} \ldots a_{D-2}}
$$

where $\epsilon_{a_{1} \ldots a_{D-2}}$ is the unperturbed intrinsic volume form on $B$. From the definition, eq. (18), of $v^{a}$ we have on $B$

$$
\begin{aligned}
v^{a} n_{a} & =g^{f h} n^{e} \nabla_{f} \delta g_{h e}-g^{h e} n^{f} \nabla_{f} \delta g_{h e} \\
& =-g^{f h} n^{e} \nabla_{f}\left(2 r \nabla_{(h} u \delta \beta_{e)}\right)+g^{e h} n^{f} \nabla_{f}\left(2 r \nabla_{(h} u \delta \beta_{e)}\right)+g^{f h} n^{e} \nabla_{f} \delta \mu_{a b}-g^{e h} n^{f} \nabla_{f} \delta \mu_{a b} .
\end{aligned}
$$

The first two terms on the right hand side can give a non-zero result on $B$ (i.e. $r=0$ ) only if the derivative $\nabla_{f}$ acts on $r$, but in that case the tensor contractions are seen to give a zero result. For the last two terms on the right side, we get, again on $B$,

$$
\begin{aligned}
v^{a} n_{a} & =2 n^{(f} l^{h)} n^{e} \nabla_{f} \delta \mu_{h e}-2 n^{(e} l^{h)} n^{f} \nabla_{f} \delta \mu_{h e}+\mu^{f h} n^{e} \nabla_{f} \delta \mu_{h e}-\mu^{e h} n^{f} \nabla_{f} \delta \mu_{h e} \\
& =-\mu^{e h} n^{f} \nabla_{f} \delta \mu_{h e}-\mu^{f h}\left(\nabla_{f} n^{e}\right) \delta \mu_{h e} \\
& =-\mu^{a b} \mathscr{L}_{n} \delta \mu_{a b}+\frac{1}{2} \delta \mu^{a b} \mathscr{L}_{n} \mu_{a b} \\
& =-\mu^{a b} \mathscr{L}_{n} \delta \mu_{a b}=-2 \delta \vartheta .
\end{aligned}
$$


Here, we have used in the first line the expression for $g_{a b}$ in gaussian null coordinates (5), whereas to go to the second line we used that at least one vector $n^{a}, l^{a}$ gets contracted into $\delta \mu_{a b}$ when all vectors are pulled through the derivative, and this gives a zero result. To go to the third line we used standard expressions for the Lie-derivative of a tensor, and that $\mu^{a}{ }_{c} \mu^{b}{ }_{d} \nabla^{c} n^{d}=\frac{1}{2} \mathscr{L}_{n} \mu_{a b}$ on $B$, which follows from (5). To go to the fourth line we used that $\mathscr{L}_{n} \mu_{a b}=0$, as the background is stationary. Thus, we have shown that

$$
\int_{B} i_{\xi} \theta=-\frac{1}{8 \pi} \int_{B} f \delta \vartheta \epsilon
$$

Next, we calculate $\delta Q_{\xi}$. When pulled back to $B$, we have

$$
\left(\delta Q_{\xi}\right)_{a_{1} \ldots a_{D-2}}=\frac{1}{16 \pi} n^{a} l^{b} \nabla_{[a}\left(\delta g_{b] c} \xi^{c}\right) \epsilon_{a_{1} \ldots a_{D-2}}+\frac{1}{32 \pi} n^{a} l^{b} \nabla_{[a} \xi_{b]} \delta g_{c}^{c} \epsilon_{a_{1} \ldots a_{D-2}} .
$$

Since $\delta g_{a b} \xi^{b}=u \delta \mu_{a b} Z^{b}+r \delta \mu_{a b} Y^{b}$, we have on $B$,

$$
\begin{aligned}
2 n^{a} l^{b} \nabla_{[a}\left(\delta g_{b] c} \xi^{c}\right) & =n^{a} l^{b}\left(\nabla_{a} u \delta \mu_{b c} Z^{c}-\nabla_{b} u \delta \mu_{a c} Z^{c}+\nabla_{a} r \delta \mu_{b c} Y^{c}-\nabla_{b} r \delta \mu_{a c} Y^{c}\right) \\
& =l^{b} \delta \mu_{b c} Z^{c}+n^{b} \delta \mu_{b c} Y^{c}=0
\end{aligned}
$$

as $\delta \mu_{a b} n^{a}=0=\delta \mu_{a b} l^{a}$. Thus, the first term on the right side of $\left.\delta Q_{\xi}\right|_{B}$ is zero. To calculate the second term, we use, on $B$,

$$
\begin{aligned}
& 2 n^{a} l^{b} \nabla_{[a} \xi_{b]} \\
= & n^{a} l^{b}\left[\left(\nabla_{a} f\right) n_{b}+\left(\nabla_{a} u\right) Z^{b}+\left(\nabla_{a} r\right) Y_{b}-\left(\nabla_{b} f\right) n_{a}-\left(\nabla_{b} u\right) Z_{a}-\left(\nabla_{b} r\right) Y_{a}+2 f \nabla_{[a} n_{b]}\right] \\
= & n^{a} Y_{a}+l^{a} Z_{a}+n^{a} \nabla_{a} f+\frac{1}{2} f l^{a} \nabla_{a}\left(n^{b} n_{b}\right)-f l_{b} n^{a} \nabla_{a} n^{b} \\
= & l^{a} Z_{a}+n^{a} Y_{a}+n^{a} \nabla_{a} f
\end{aligned}
$$

as $n^{a} \nabla_{a} r=n^{a} n_{a}=O(r)$, and as $\nabla_{b}\left(n^{a} n_{a}\right)=\nabla_{b}\left(r^{2} \alpha\right)=O(r)$, and $n^{a} \nabla_{a} n^{b}=O(r)$ since $n^{a}$ is tangent to affinely parameterized null geodesics on $\mathscr{H}^{+}$. Thus, we obtain

$$
\int_{B} \delta Q_{\xi}=\frac{1}{64 \pi} \int_{B}\left(n^{a} Y_{a}+l^{a} Z_{a}+n^{a} \nabla_{a} f\right) \delta \mu_{b}^{b} \epsilon
$$

Thus, combining this with the previous result, we have shown that, with $\xi^{a}=f n^{a}+$ $u Z^{a}+r Y^{a}$ :

$$
\int_{B}\left[\delta Q_{\xi}(g)-i_{\xi} \theta(g ; \delta g)\right]=-\frac{1}{8 \pi} \int_{B}\left[f \delta \vartheta \epsilon-\frac{1}{4}\left(n^{a} Y_{a}+l^{a} Z_{a}+n^{a} \nabla_{a} f\right) \delta \epsilon\right],
$$

and, hence,

$$
W_{\Sigma}\left(g ; \delta g, \mathscr{L}_{\xi} g\right)=\frac{1}{8 \pi} \int_{B}\left[f \delta \vartheta \epsilon-\frac{1}{4}\left(n^{a} Y_{a}+l^{a} Z_{a}+n^{a} \nabla_{a} f\right) \delta \epsilon\right]+c \delta H_{X}
$$

It follows immediately from this formula that if $\delta g_{a b}$ is a solution for which $\left.\delta \epsilon\right|_{B}=0=\left.\delta \vartheta\right|_{B}$ and $\delta H_{X}=0$, then $W_{\Sigma}\left(g ; \delta g, \mathscr{L}_{\xi} g\right)=0$. Conversely, if $W_{\Sigma}\left(g ; \delta g, \mathscr{L}_{\xi} g\right)=0$ for all $\xi^{a}$ of compact support, it follows immediately from (34) with $E=0$ that $\delta g_{a b}$ satisfies the linearized constraints. It then follows immediately from (159) that if $W_{\Sigma}\left(g ; \delta g, \mathscr{L}_{\xi} g\right)=0$ for all $f, Z^{a}, Y^{a}, c$, then $\left.\delta \epsilon\right|_{B}=\left.\delta \vartheta\right|_{B}=\delta H_{X}=0$. 


\section{B Proof of lemmas 3 and 4}

Lemma 3. Let $Y=\left(N_{0}, N_{0}^{a}\right) \in C_{0}^{\infty} \oplus C_{0}^{\infty}$. Then there exists a solution $X$ in the space $\cap_{k}\left(\rho^{2} W_{\rho}^{k+1}(\Sigma) \oplus \rho W_{\rho}^{k}(\Sigma)\right) \subset C^{\infty}(\bar{\Sigma}) \oplus C^{\infty}(\bar{\Sigma})$ to the following boundary value problem:

$$
\mathcal{L} \mathcal{L}^{*}(X)=Y \quad \text { in } \Sigma,
$$

and $X=\left(N, N^{a}\right)$ satisfies

$$
N^{a}=N \eta^{a}, \quad \delta \vartheta=\delta \epsilon=0 \quad \text { on } B=\partial \Sigma .
$$

Furthermore, if $D \geq 5$, the solution is unique, whereas for $D=4$, the solution is also unique unless $t^{a}$ is tangent to the generators of the horizon (i.e., the black hole is nonrotating), in which case the solution is unique up to $X^{a} \rightarrow X^{a}+c t^{a}$.

Proof: The key ingredient in the proof is a Poincare-type inequality for certain tensor fields $X=\left(N, N^{a}\right) \in W_{\rho}^{2} \oplus W_{\rho}^{1}$. Tensor fields $X \in W_{\rho}^{2} \oplus W_{\rho}^{1}$ have, by the usual theorems about Sobolev spaces, a well defined restriction to $B$ in the space $W^{3 / 2}(B) \oplus W^{1 / 2}(B)$, and the restriction map is continuous. Consequently, the space

$$
\mathscr{X}:=\left\{X=\left(N, N^{a}\right) \in W_{\rho}^{2} \oplus W_{\rho}^{1} \quad \mid \quad N^{a}=N \eta^{a} \quad \text { on } B\right\}
$$

is a closed subspace of $W_{\rho}^{2} \oplus W_{\rho}^{1}$. We first give the proof for $D \geq 5$ and then give the modifications to the proof for $D=4$.

$\underline{D \geq 5:}$ For $X \in \mathscr{X}$, we claim the following inequality in $D \geq 5$ :

$$
C\|X\|_{W_{\rho}^{2} \oplus W_{\rho}^{1}} \geq\left\|\mathcal{L}^{*} M_{\rho}(X)\right\|_{L^{2} \oplus L^{2}} \geq c\|X\|_{W_{\rho}^{2} \oplus W_{\rho}^{1}}, \quad M_{\rho}=\left(\begin{array}{cc}
\rho^{2} & 0 \\
0 & \rho
\end{array}\right),
$$

for non-zero constants $c, C$. The key inequality is the second one; the first inequality is merely a straightforward consequence of the definition of $\mathcal{L}^{*}$, together with the asymptotic conditions (27) on the background spacetime and the fact that $D^{a} \rho D_{a} \rho \leq$ $C, \rho^{2} D^{a} D^{b} \rho D_{b} D_{b} \rho \leq C$, for other constants $C$. Combining the equality (163) in the usual way with the Lax-Milgram theorem [17], we then infer the existence of $\tilde{X} \in \mathscr{X}$ satisfying

$$
0=\left\langle\mathcal{L}^{*} M_{\rho} \tilde{X} \mid \mathcal{L}^{*} M_{\rho} \tilde{\psi}\right\rangle_{\mathcal{K}}+\langle\tilde{Y} \mid \tilde{\psi}\rangle_{\mathcal{K}} \quad \text { for all } \tilde{\psi} \in \mathscr{X}
$$

for any source $\tilde{Y} \in C_{0}^{\infty} \oplus C_{0}^{\infty}$ in dimensions $D \geq 5$. Thus, taking $\tilde{Y}=M_{\rho} Y$, and $X=M_{\rho} \tilde{X}$, we get a distributional solution $X$ to our equation (160) in the space $M_{\rho} \mathscr{X}$.

The solution is actually more regular, because, as is well-known [4, 6], the operator $\mathcal{L} \mathcal{L}^{*}$ is an elliptic matrix operator of mixed type in the sense of Agmon-Douglis-Nirenberg; the leading derivative terms ("principal symbol") are in fact $\sigma_{\mathcal{L L}^{*}}=\sigma_{\mathcal{L}^{*}}{ }^{T} \sigma_{\mathcal{L}^{*}}$, with $X^{a}=$ $\left(N, N^{a}\right)$ and

$$
16 \pi \sigma_{\mathcal{L}^{*}}\left(\begin{array}{c}
N \\
N^{c}
\end{array}\right)=\left(\begin{array}{cc}
\xi_{a} \xi_{b}-h_{a b} \xi_{d} \xi^{d} & h^{-\frac{1}{2}}\left(-p_{a b} \xi_{c}+2 \xi_{d} h_{c(a} p_{b)}{ }^{d}\right) \\
2 p^{a b}-\frac{2}{D-2} h^{a b} p & 2 h^{\frac{1}{2}} \delta^{(b}{ }_{c} \xi^{a)}
\end{array}\right)\left(\begin{array}{c}
N \\
N^{c}
\end{array}\right)
$$

As a consequence, the standard techniques for elliptic operators (as adapted to systems of mixed type such as ours, see p. 210 of [28], and taking into account the weight factors of $\rho$ ) 
then give that the solutions $\tilde{X}$ are in fact in the Sobolev spaces $\tilde{X} \in W_{\rho}^{k+4} \oplus W_{\rho}^{k+2}$ for all $k$, away from the boundary of $\Sigma$. Actually, this regularity extends to the boundary $\partial \Sigma=B$ by thm. 6.1 of [34, which is applicable37 in view of (163). By standard embedding theorems for Sobolev spaces, $\tilde{X}$, and also $X$, are in $C^{\infty}(\bar{\Sigma}) \oplus C^{\infty}(\bar{\Sigma})$. It is then also straightforward to see that $X$ solves the desired boundary value problem. First, because $\tilde{X} \in \mathscr{X}$, we have $\tilde{N}^{a}=\tilde{N} \eta^{a}$, and because $\rho$ is constant near $B$, the same holds for $X^{a}$. Thus, we satisfy the first of the required boundary conditions. Next, because $X$ is already known to be a weak solution of the equation (160), and because it is smooth, it must satisfy the equation in the classical sense. Furthermore, performing a partial integration in eq. (164), with $X=M_{\rho} \tilde{X}, Y=M_{\rho}^{-1} \tilde{Y}$, and smooth $\tilde{\psi}$ compactly supported in $\bar{\Sigma}$, then shows that be boundary terms must vanish. The boundary terms are obtained from (78). Because $\psi$ has compact support in $\bar{\Sigma}$, only a boundary term from $B$ arises, which, with $(\delta h, \delta p):=\mathcal{L}^{*} M_{\rho} \tilde{X}$, is given by $0=\int_{B}\left[\delta Q_{\psi}(g)-i_{\psi} \theta(\delta g)\right]$, where $\psi=M_{\rho} \tilde{\psi}$. In view of eq. (158) this implies that $\left.\delta \vartheta\right|_{B}=0=\left.\delta \epsilon\right|_{B}$. These are precisely the last two remaining boundary conditions in (161).

Thus, what remains is to demonstrate the weighted Poincare-inequality (163). We first consider smooth $X$ with compact support in a compact set $K \subset \bar{\Sigma}$, where $\rho$ may be assumed to be 1 . In other words, such $X$ are zero in the asymptotic region, but not necessarily on $B$. For such fields, we can find a constant $c>0$ such that

$$
c\|X\|_{W^{2} \oplus W^{1}} \leq\left\|\mathcal{L}^{*}(X)\right\|_{L^{2} \oplus L^{2}}+\|X\|_{W^{1} \oplus W^{0}}+\left|\int_{B} h^{\frac{1}{2}}\left(D_{b} N_{a} N^{b}-D_{b} N^{b} N_{a}\right) \eta^{a}\right|^{\frac{1}{2}}
$$

For a proof, see lemma 2.6 of [6]. Now we assume that $N^{a}=N \eta^{a}$ on $B$. This means that, near $B$, we can write $N^{a}=N \eta^{a}+x Y^{a}$, with $x=\operatorname{dist}_{B}$ the geodesic distance to $B$, and $Y^{a}$ some smooth tensor field. Then the boundary term becomes

$$
\begin{aligned}
& \int_{B} h^{\frac{1}{2}}\left(D_{b} N_{a} N^{b}-D_{b} N^{b} N_{a}\right) \eta^{a} \\
= & \int_{B} h^{\frac{1}{2}}\left(N D_{b} N \eta^{b}+N^{2} \eta^{a} \eta^{b} D_{b} \eta^{a}+N Y_{a} \eta^{a}-N D_{b} N \eta^{b}-N^{2} D_{a} \eta^{a}-N Y_{a} \eta^{a}\right) \\
= & -\int_{B} N^{2} H h^{\frac{1}{2}}=0,
\end{aligned}
$$

where $H$ is the trace of the extrinsic curvature of $B$ inside $\Sigma$. To simplify the calculation, we have assumed that $\eta^{a}$ is extended away from $B$ such that $\eta^{a} D_{a} \eta^{b}=0$, and we used $D_{a} x=\eta_{a}$. Because $B$ is the bifurcation surface, $H=0$, and the boundary term vanishes. Hence, (166) holds without the boundary term if $X$ satisfies $N^{a}=N \eta^{a}$ on $B$, i.e. if $X \in \mathscr{X} \cap\left(C_{0}^{\infty}(K) \oplus C_{0}^{\infty}(K)\right)$.

Consider next an open region $O \subset \Sigma$ such that $\bar{O} \subset K$. It is shown combining Prop. 7.2 and Lemma 4.2 of [6], that for all $X \in C_{0}^{\infty}(\Sigma \backslash O) \oplus C_{0}^{\infty}(\Sigma \backslash O)$, we have

$$
c\|X\|_{W^{2} \oplus W^{1}} \leq\left\|\mathcal{L}^{*} M_{\rho}(X)\right\|_{L^{2} \oplus L^{2}}
$$

for some constant $c>0$. We now combine this and eq. (166) (without boundary term) to obtain the desired inequality (163). Suppose, to obtain a contradiction, that there

\footnotetext{
${ }^{37}$ Note that the arguments in this paper are entirely local near $B$, so it does not matter that our domain is not bounded.
} 
is a sequence $X_{n} \in \mathscr{X}$, such that $\left\|X_{n}\right\|_{W_{\rho}^{2} \oplus W_{\rho}^{1}}=1$, but $\left\|\mathcal{L}^{*} M_{\rho}\left(X_{n}\right)\right\|_{L^{2} \oplus L^{2}} \rightarrow 0$. Let $\chi_{1}+\chi_{2}=1$ be a partition of unity such that $\operatorname{supp} \chi_{1} \subset K, \operatorname{supp} \chi_{2} \subset \Sigma \backslash O$. Then we estimate

$$
\begin{aligned}
\left\|X_{n}\right\|_{W_{\rho}^{2} \oplus W_{\rho}^{1}} \leq & \left\|\chi_{1} X_{n}\right\|_{W_{\rho}^{2} \oplus W_{\rho}^{1}}+\left\|\chi_{2} X_{n}\right\|_{W_{\rho}^{2} \oplus W_{\rho}^{1}} \\
\leq & C\left\|\mathcal{L}^{*}\left(\chi_{1} X_{n}\right)\right\|_{L^{2} \oplus L^{2}}+C\left\|\chi_{1} X_{n}\right\|_{W^{1} \oplus W^{0}}+C\left\|\mathcal{L}^{*} M_{\rho}\left(\chi_{2} X_{n}\right)\right\|_{L^{2} \oplus L^{2}} \\
\leq & C\left\|\chi_{1} \mathcal{L}^{*}\left(X_{n}\right)\right\|_{L^{2} \oplus L^{2}}+C\left\|\left[\mathcal{L}^{*}, \chi_{1}\right] X_{n}\right\|_{L^{2} \oplus L^{2}}+C \chi_{1} X_{n} \|_{W^{1} \oplus W^{0}}+ \\
& C\left\|\chi_{2} \mathcal{L}^{*} M_{\rho}\left(X_{n}\right)\right\|_{L^{2} \oplus L^{2}}+C\left\|\left[\mathcal{L}^{*}, \chi_{2}\right] M_{\rho} X_{n}\right\|_{L^{2} \oplus L^{2}} \\
\leq & C\left\|\mathcal{L}^{*} M_{\rho}\left(X_{n}\right)\right\|_{L^{2}(\Sigma) \oplus L^{2}(\Sigma)}+C\left\|X_{n}\right\|_{W^{1}(O) \oplus W^{0}(O)}
\end{aligned}
$$

with possibly new constants in each line. In the last step we used that the commutator $\left[\mathcal{L}^{*}, \psi\right]$ with a smooth compactly supported function $\psi$ decreases the order of each entry of the matrix operator $\mathcal{L}^{*}$ by one unit (unless the order of the entry is already $=0$ ), so that $\left[\mathcal{L}^{*}, \psi\right]: W^{2} \oplus W^{1} \rightarrow W^{1} \oplus W^{0}$ is bounded. Now, by assumption, $\left\|\mathcal{L}^{*} M_{\rho}\left(X_{n}\right)\right\|_{L^{2} \oplus L^{2}} \rightarrow 0$ for the first term on the right side. On the other hand, since $X_{n}$ is by assumption bounded in $W^{2}(O) \oplus W^{1}(O)$, it follows from the Rellich-Kondrachov compactness theorem (see e.g. [17]) that $X_{n}$ (or a subsequence thereof) is Cauchy in $W^{1}(O) \oplus W^{0}(O)$. Hence, the above inequality shows that a subsequence of $X_{n}$ is Cauchy in $\mathscr{X} \subset W_{\rho}^{2}(\Sigma) \oplus W_{\rho}^{1}(\Sigma)$, hence convergent with limit $X \in \mathscr{X}$. Since the norm of $X_{n}$ in this space is $=1, X \neq 0$, and by the continuity of $\mathcal{L}^{*} M_{\rho}: \mathscr{X} \rightarrow L^{2} \oplus L^{2}$, we learn that $\xi:=M_{\rho} X \in \rho^{2} W_{\rho}^{2} \oplus \rho W_{\rho}^{1}$ satisfies $\mathcal{L}^{*}(\xi)=0$. So $\xi^{a}$ must be equal, almost everywhere, to a non-trivial Killing vector field. However, a nontrivial Killing field in an asymptotically flat spacetime must approach an infinitesimal Poincare transformation near infinity. Consequently, in $D \geq 5$ spacetime dimensions, there are no non-zero Killing for which $M_{\rho}^{-1} \xi \in L^{2} \oplus L^{2}$, hence a contradiction.

$\underline{D=4}$ : In this dimension, the last statement is not true for the timelike Killing field $\xi^{a}=t^{a}$ if the background is such that $t^{a}=\left(N, N^{a}\right)$ is tangent to the generators of the horizon: Indeed, $M_{\rho}^{-1} t$ then is in $L^{2} \oplus L^{2}$, and it satisfies the boundary condition $N^{a}=N \eta^{a}$. Consequently, the Poincare inequality (163) is not true in $D=4$ e.g. for a Schwarzschild background. However, in that case we can modify our argument as follows. We now supplement the definition of the space $\mathscr{X}$ eq. (162) by the condition that elements of $\mathscr{X}$ should be $L^{2} \oplus L^{2}$ orthogonal to $M_{\rho}^{-1} t$. Then, in this space, the Poincare inequality holds, and the rest of the argument goes through, giving a unique solution to $\mathcal{L}^{*} \mathcal{L}(X)=Y$ in $M_{\rho} \mathscr{X}$ with the desired boundary conditions. The general solution is then simply $X^{a}+c t^{a}$.

Lemma 4. Let $j^{a}$ be a smooth vector field on $\Sigma$ such that $j^{a} \in \rho^{-1} W_{\rho}^{k-1}$ for any $k>0$. Then there exists a unique, solution $X^{a} \in \rho W_{\rho}^{k}$ to the boundary value problem

$$
D_{a}\left(D^{(a} X^{b)}-\frac{1}{D-1} h^{a b} D_{c} X^{c}\right)=j^{b}
$$

and

$$
\left.\left(h^{a b}-\eta^{a} \eta^{b}\right) X_{a}\right|_{B}=0,\left.\quad \eta^{a} D_{a}\left(\eta^{b} X_{b}\right)\right|_{B}=0
$$


Furthermore $\left\|\rho^{-1} X^{a}\right\|_{W_{\rho}^{k}} \leq C\left\|\rho j^{a}\right\|_{W_{\rho}^{k-1}}$.

The same statement is true for the boundary value problem for $\tilde{X}^{a}$ on the slice $\tilde{\Sigma}=$ $\Sigma \times \mathbb{T}^{p}$ in the black brane spacetime with spatial metric $\tilde{h}_{a b}=h_{a b}+\sum\left(d z_{i}\right)_{a}\left(d z_{i}\right)_{b}$, with $D_{a}$ replaced by $\tilde{D}_{a}$, and with the coefficient in the trace term in the operator replaced by $1 /(D+p-1)$.

Proof: Define $(P X)_{a b}=D_{(a} X_{b)}-\frac{1}{D-1} h_{a b} D^{c} X_{c}$. The key ingredient of the proof of is the weighted Poincare inequality

$$
c \int_{\Sigma} \rho^{-2} X_{a} X^{a} h^{\frac{1}{2}} \leq \int_{\Sigma}(P X)^{a b}(P X)_{a b} h^{\frac{1}{2}}
$$

for some $c>0$. This inequality holds for any smooth $X^{a}$ satisfying the boundary conditions stated in lemma 4. In order to prove this inequality, we consider the identity

$$
\begin{aligned}
& (P X)^{a b}(P X)_{a b}= \\
& \frac{1}{2}\left(D_{a} X_{b}\right) D^{a} X^{b}+D_{a}\left(X^{[b} D_{b} X^{a]}\right)-\frac{1}{2} R_{a b}(h) X^{a} X^{b}+\frac{D-3}{2(D-1)}\left(D_{c} X^{c}\right)^{2},
\end{aligned}
$$

which holds for any vector field. Now let $O=\{\rho<C\}$ for some large $C$, and assume that $X^{a}$ has compact support in $\bar{\Sigma} \backslash O$. Integrating the above identity then gives

$$
\begin{aligned}
\frac{1}{2} \int_{\Sigma}\left(D_{a} X_{b}\right) D^{a} X^{b} h^{\frac{1}{2}} & \leq \int_{\Sigma}(P X)^{a b}(P X)_{a b} h^{\frac{1}{2}}+\frac{1}{2} \int_{\Sigma} R_{a b}(h) X^{a} X^{b} h^{\frac{1}{2}} \\
& \leq \int_{\Sigma}(P X)^{a b}(P X)_{a b} h^{\frac{1}{2}}+\frac{1}{2} \sup _{\bar{\Sigma} \backslash O} \rho^{2}\left(R_{a b}(h) R^{a b}(h)\right)^{\frac{1}{2}} \int_{\Sigma} \rho^{-2} X^{a} X_{a} h^{\frac{1}{2}} \\
& \leq \int_{\Sigma}(P X)^{a b}(P X)_{a b} h^{\frac{1}{2}}+\epsilon \int_{\Sigma} \rho^{-2} X^{a} X_{a} h^{\frac{1}{2}}
\end{aligned}
$$

for any given $\epsilon>0$, provided $C$ is chosen sufficiently large (here we use that $R_{a b}(h)=$ $\left.O\left(\rho^{-(D-1)}\right)\right)$. Now from prop. C.5 of [6], we also have for sufficiently large $C$

$$
c \int_{\Sigma} \rho^{-2} X^{a} X_{a} h^{\frac{1}{2}} \leq \int_{\Sigma}\left(D_{a} X_{b}\right) D^{a} X^{b} h^{\frac{1}{2}}
$$

and some $c>0$. It follows that inequality (172) holds if $X^{a}$ is supported outside a sufficiently large $O$. Consider next a compact set $K$ such that $K \supset \bar{O}$, and let $X^{a}$ now be smooth and supported in $K$. Integrating now our identity (173) over $K$, we obtain

$$
c\|X\|_{W^{1}} \leq\|P X\|_{L^{2}}+\|X\|_{L^{2}}+\left|\int_{B} h^{\frac{1}{2}}\left(D_{b} X_{a} X^{b}-D_{b} X^{b} X_{a}\right) \eta^{a}\right|^{\frac{1}{2}}
$$

for some $c>0$. Let now $\mathscr{X}=\left\{X^{a} \in \rho W_{\rho}\left|0=\left(h^{a b}-\eta^{a} \eta^{b}\right) X_{b}\right|_{B}\right\}$. Then the same calculation as in (167) shows that the boundary term vanishes for $X^{a} \in \mathscr{X}$. Thus, for $X^{a} \in \mathscr{X}$ we have eq. (172) if the support of $X^{a}$ is outside $O$, whereas we have (176) without boundary term if the support of $X^{a}$ is in $K$. By considering now a partition 
of unity for the covering $O \cup K=\bar{\Sigma}$, and arguing precisely as above around (169), we conclude that the Poincare inequality (172) holds for any $X^{a} \in \mathscr{X}$, unless there is an $X^{a} \in \mathscr{X}$ such that $(P X)_{a b}=0$. By the definition of $P$, such a $X^{a}$ would be a conformal Killing vector field for $h_{a b}$, and would hence have to approach a conformal Killing vector field on $\mathbb{R}^{D-1}$ in the asymptotic region. In particular, the slowest possible fall-off of $X^{a}$ would be that of a translation. However, by the definition of $\mathscr{X}$, we would also have to have $\rho^{-1} X^{a} \in L^{2}$, which is impossible. This establishes that the Poincare inequality holds for any $X^{a} \in \mathscr{X}$.

Combining the Poincare inequality in the usual way with the Lax-Milgram theorem on $\mathscr{X}$ (see lemma 3 for a similar argument), we get a weak solution $X^{a} \in \mathscr{X}$ to our boundary value problem in the weak sense, i.e. $\langle P \psi \mid P X\rangle=-\langle\psi \mid j\rangle$ for all $\psi^{a} \in \mathscr{X}$. This also gives $\left\|\rho^{-1} X\right\|_{W_{\rho}^{1}} \leq C\|\rho j\|_{L^{2}}$. The usual bootstrap and scaling arguments then show $\left\|\rho^{-1} X\right\|_{W_{\rho}^{k}} \leq C\|\rho j\|_{W_{\rho}^{k-1}}$ for any $k$, so the weak solution is a strong solution satisfying the Neumann condition $\left.\eta^{a} D_{a}\left(\eta^{b} X_{b}\right)\right|_{B}=0$, whereas the remaining boundary conditions are already built into $\mathscr{X}$. This completes the proof of the lemma in the asymptotically flat case. The asymptotically KK case is exactly the same.

\section{Strengthened version of property (3) of Proposi- tion 5}

In this Appendix, we strengthen property (3) of proposition 5 with the following lemma:

Lemma 5. Let $(\delta h, \delta p)$ be a perturbation in the dense subspace $\mathcal{V} \cap\left(W_{\rho}^{k+1} \oplus W_{\rho}^{k}\right)$ for sufficiently large $k$. Then for any $\delta>0$, we can find a new perturbation $\left(\delta h^{\prime}, \delta p^{\prime}\right)$ in the same subspace having the property that it vanishes in a neighborhood of spatial infinity, and is such that $\left\|(\delta h, \delta p)-\left(\delta h^{\prime}, \delta p^{\prime}\right)\right\|_{W_{\rho}^{k+1} \oplus W_{\rho}^{k}}<\delta$. In fact, if $(\delta h, \delta p) \in \mathcal{V} \cap\left(C^{\infty}(\bar{\Sigma}) \oplus\right.$ $\left.C^{\infty}(\bar{\Sigma})\right)$, then $\left(\delta h^{\prime}, \delta p^{\prime}\right)$ can be chosen in $\mathcal{V} \cap\left(C_{0}^{\infty}(\bar{\Sigma}) \oplus C_{0}^{\infty}(\bar{\Sigma})\right)$, i.e. smooth and vanishing near spatial infinity.

Proof: The proof of this lemma follows from the (much more general) techniques and results in [4, 5], and also [6]. We consider a sufficiently large $R>0$ and the corresponding annular domain $\Omega=\{p \in \Sigma \mid R<\rho(p)<2 R\}$, with $\rho>0$ as usual a function which is equal to the radial distance in the asymptotic region. Furthermore, we introduce a function $x: \Omega \rightarrow \mathbb{R}$ which in a small neighborhood of $\partial \Omega$ is given by $x=\operatorname{dist}_{\partial \Omega}$, and satisfies $x>0$ in the interior. A new norm for functions or tensor fields $u \in C_{0}^{\infty}(\Omega)$ is then introduced by

$$
\|u\|_{W_{0, \rho x^{2}, \mathrm{e}^{-1 / x}}^{k}}:=\left\{\sum_{n=0}^{k} \int_{\Sigma} h^{\frac{1}{2}}\left(D_{\left(a_{1}\right.} \cdots D_{\left.a_{n}\right)} u\right) D^{\left(a_{1}\right.} \cdots D^{\left.a_{n}\right)} u\left(\rho x^{2}\right)^{2 n} \mathrm{e}^{-2 / x}\right\}^{\frac{1}{2}}
$$

and $W_{0, \rho x^{2}, \mathrm{e}^{-1 / x}}^{k}$ is defined to be the closure of such functions or tensor fields in this norm. Now let $\chi$ be a smooth function which is equal to 1 in the interior of $\Sigma$, which interpolates between 1 and 0 strictly inside $\Omega$, and which is 0 for $\rho>2 R$. We can choose this function so that $\left(D_{a_{1}} \ldots D_{a_{n}} \chi\right) D^{a_{1}} \ldots D^{a_{n}} \chi \leq C R^{-2 n}$. Our first try for the compactly supported initial data is $(\chi \delta h, \chi \delta p)$. Then for each $\delta>0, k \in \mathbb{N}$ we can find a sufficiently large $R$ so that 
$\|(\delta h, \delta p)-(\chi \delta h, \chi \delta p)\|_{W_{\rho}^{k+1} \oplus W_{\rho}^{k}}<\delta$. Of course, $(\chi \delta h, \chi \delta p)$ does not satisfy the linearized constraints; $\mathcal{L}(\chi \delta h, \chi \delta p)=: Y$ is a non-zero element $Y \in W_{0, \rho x^{2}, \mathrm{e}^{-1 / x}}^{k-1}(\Omega) \oplus W_{0, \rho x^{2}, \mathrm{e}^{-1 / x}}^{k-1}(\Omega)$ (with the usual decomposition of $Y^{a}$ into lapse and shift understood). To satisfy the linearized constraints, we wish to correct $(\chi \delta h, \chi \delta p)$ by a suitable element of the form $-\mathrm{e}^{-2 / x} M_{x}^{2} \mathcal{L}^{*}(X)$, with $M_{x}=\operatorname{diag}\left(x^{2}, x\right)$ as above, i.e. we wish to set

$$
\left(\delta h^{\prime}, \delta p^{\prime}\right):=(\chi \delta h, \chi \delta p)-\mathrm{e}^{-2 / x} M_{x}^{2} \mathcal{L}^{*}(X)
$$

The factor $\mathrm{e}^{-2 / x}$ is inserted in order that we can continue the second term sufficiently smoothly by 0 to all of $\Sigma$. Such a perturbation is then clearly compactly supported, and, to satisfy the linearized constraints, $X$ would have to satisfy

$$
\mathrm{e}^{2 / x} \mathcal{L} \mathrm{e}^{-2 / x} M_{x}^{2} \mathcal{L}^{*}(X)=\mathrm{e}^{2 / x} Y
$$

The operator $\mathrm{e}^{2 / x} \mathcal{L} \mathrm{e}^{-2 / x} M_{x}^{2} \mathcal{L}^{*}$ on the left hand side is shown in [6] to be a bounded map

$$
\mathfrak{k}^{\perp} \cap\left(W_{0, \rho x^{2}, \mathrm{e}^{-1 / x}}^{k+4}(\Omega) \oplus W_{0, \rho x^{2}, \mathrm{e}^{-1 / x}}^{k+2}(\Omega)\right) \rightarrow \mathfrak{k}^{\perp} \cap\left(W_{0, \rho x^{2}, \mathrm{e}^{-1 / x}}^{k}(\Omega) \oplus W_{0, \rho x^{2}, \mathrm{e}^{-1 / x}}^{k}(\Omega)\right)
$$

where $\mathfrak{k}=\operatorname{span}\left(t^{a}, \psi_{1}^{a}, \ldots, \psi_{N}^{a}\right)$ is the span of the Killing fields, and $\perp$ means orthogonal complement in $L^{2}\left(\Omega, \mathrm{e}^{-2 / x} h^{\frac{1}{2}}\right)$. Furthermore, the operator has a bounded inverse. It follows from $\delta M, \delta J_{A}=0$ for the perturbation $(\delta h, \delta p) \in \mathcal{V}$ [cf. Proposition 5 ] that

$$
\begin{aligned}
& \left\langle\xi \mid \mathrm{e}^{2 / x} Y\right\rangle_{L^{2}\left(\Omega, h^{\frac{1}{2}} \mathrm{e}^{-2 / x}\right)}=\langle\xi \mid \mathcal{L}(\chi \delta h, \chi \delta p)\rangle_{L^{2}(\Sigma)}= \\
& \left\langle\mathcal{L}^{*}(\xi) \mid Y\right\rangle_{L^{2}(\Sigma) \oplus L^{2}(\Sigma)}-\delta H_{\xi}(\delta h, \delta p)=0+0
\end{aligned}
$$

for any $\xi \in \mathfrak{k}$. Hence $\mathrm{e}^{2 / x} Y$ is in $\mathfrak{k}^{\perp}$. Furthermore, for sufficiently large $R$, the $W_{0, \rho x^{2}, \mathrm{e}^{-1 / x}}^{k}(\Omega) \oplus$ $W_{0, \rho x^{2}, \mathrm{e}^{-1 / x}}^{k}(\Omega)$-norm is arbitrarily small. So we can invert (179) with $X \in W_{0, \rho x^{2}, \mathrm{e}^{-1 / x}}^{k+3} \oplus$ $W_{0, \rho x^{2}, \mathrm{e}^{-1 / x}}^{k+1}$ having small norm, and hence with $-\mathrm{e}^{-2 / x} M_{x}^{2} \mathcal{L}^{*}(X) \in \mathrm{e}^{-2 / x} M_{x}^{2}\left(W_{0, \rho x^{2}, \mathrm{e}^{-1 / x}}^{k+1}(\Omega) \oplus\right.$ $\left.W_{0, \rho x^{2}, \mathrm{e}^{-1 / x}}^{k}(\Omega)\right)$ having small norm in $W_{\rho}^{k+1}(\Sigma) \oplus W_{\rho}^{k}(\Sigma)$, where we extend the tensor fields by 0 outside $\Omega$. Consequently, for sufficiently large $R$, the $W_{\rho}^{k+1}(\Sigma) \oplus W_{\rho}^{k}(\Sigma)$-norm of

$$
\left(\begin{array}{l}
\delta h^{\prime} \\
\delta p^{\prime}
\end{array}\right)-\left(\begin{array}{l}
\delta h \\
\delta p
\end{array}\right)=\left(\begin{array}{c}
(\chi-1) \delta h \\
(\chi-1) \delta p
\end{array}\right)-\mathrm{e}^{-2 / x} M_{x}^{2} \mathcal{L}^{*}(X) \in W_{\rho}^{k+1}(\Sigma) \oplus W_{\rho}^{k}(\Sigma)
$$

is as small as we like. By construction $\left(\delta h^{\prime}, \delta p^{\prime}\right)$ satisfies the linearized constraints, and has $\delta M=\delta \mathbf{P}=\delta \mathbf{J}=0$ and $\left.\delta \vartheta\right|_{B}=\left.\delta \epsilon\right|_{B}=0$. Thus, it is in the space $\mathcal{V}$ by proposition 5 , As shown in prop. 5.7 of [6], if $(\delta h, \delta p) \in \mathcal{V}$ is even smooth, then $-\mathrm{e}^{-2 / x} M_{x}^{2} \mathcal{L}^{*}(X)$ is a smooth pair of tensor fields on $\Omega$, which can be continued by 0 outside of $\Omega$ and the resulting tensor fields $\left(\delta h^{\prime}, \delta p^{\prime}\right)$ are in $C_{0}^{\infty}(\bar{\Sigma})$.

\section{References}

[1] L. Andersson, M. Mars and W. Simon, "Local existence of dynamical and trapping horizons," Phys. Rev. Lett. 95, 111-102 (2005) [gr-qc/0506013].

[2] G. A. Burnett and R. M. Wald: "A conserved tensor for perturbations of EinsteinMaxwell systems," Proc. R. Soc. Lond. A 430, no. 1878 57-67 (1990) 
[3] P. T. Chrusciel and R. M. Wald, "Maximal Hypersurfaces in Stationary, Asymptotically Flat Spacetimes," Commun. Math. Phys. 163, 561-604 (1994).

[4] J. Corvino, Scalar curvature deformation and a gluing construction for the Einstein constraint equations, Commun. Math. Phys. 214 (2000), 137-189.

[5] J. Corvino and R. M. Schoen, "On the asymptotics for the vacuum Einstein constraint equations," gr-qc/0301071.

[6] P. T. Chrusciel and E. Delay, "On mapping properties of the general relativistic constraints operator in weighted function spaces, with applications," Mem. Soc. Math. France 94, 1 (2003) gr-qc/0301073].

[7] O. J. C. Dias, P. Figueras, R. Monteiro, H. S. Reall and J. E. Santos, "An instability of higher-dimensional rotating black holes," JHEP 1005, 076 (2010) arXiv:1001.4527 [hep-th]].

[8] M. Durkee and H. S. Reall, "Perturbations of higher-dimensional spacetimes," Class. Quant. Grav. 28, 035011 (2011) arXiv:1009.0015 [gr-qc]].

[9] M. Durkee and H. S. Reall, "Perturbations of near-horizon geometries and instabilities of Myers-Perry black holes," Phys. Rev. D 83, 104044 (2011) arXiv:1012.4805 [hep-th]].

[10] P. Figueras, K. Murata and H. S. Reall, "Black hole instabilities and local Penrose inequalities," Class. Quant. Grav. 28, 225030 (2011) [arXiv:1107.5785 [gr-qc]].

[11] J. L. Friedman, B. F. Schutz: "Gravitational radiation instability in rotating stars," Astrophys. J. 199, L157-L159 (1975)

[12] J. L. Friedman, B. F. Schutz: "Lagrangian perturbation theory of nonrelativistic fluids", Astrophys. J. 221, 937 (1978).

[13] J. L. Friedman and B. F. Schutz, "Secular instability of rotating Newtonian stars," Astrophys. J. 222, 281 (1978).

[14] J. L. Friedman: "Generic instability of rotating relativistic stars," Commun. Math. Phys. 62, 247-278 (1978).

[15] G. J. Galloway and R. Schoen, "A Generalization of Hawking's black hole topology theorem to higher dimensions," Commun. Math. Phys. 266, 571 (2006) gr-qc/0509107.

[16] R. Gregory and R. Laflamme, "Black strings and p-branes are unstable," Phys. Rev. Lett. 70, 2837 (1993) hep-th/9301052.

[17] D. Gilbarg, N. S. Trudinger: Elliptic partial differential equations of second order, Springer Grundlehren der Mathematischen Wissenschaften vol. 224 (2001)

[18] S. S. Gubser and I. Mitra, "The Evolution of unstable black holes in anti-de Sitter space," JHEP 0108, 018 (2001) hep-th/0011127]. 
[19] S. S. Gubser and I. Mitra, "Instability of charged black holes in Anti-de Sitter space," hep-th/0009126.

[20] C. X. Habisohn, "Calculation of radiated gravitational energy using the second-order Einstein tensor," J. Math. Phys. 27, 2759 (1986)

[21] S. Hollands, A. Ishibashi and R. M. Wald, "A Higher dimensional stationary rotating black hole must be axisymmetric," Commun. Math. Phys. 271, 699 (2007) gr-qc/0605106.

[22] S. Hollands and A. Ishibashi, "Asymptotic flatness and Bondi energy in higher dimensional gravity," J. Math. Phys. 46, 022503 (2005) [gr-qc/0304054|.

[23] S. Hollands and R. M. Wald, "Conformal null infinity does not exist for radiating solutions in odd spacetime dimensions," Class. Quant. Grav. 21, 5139 (2004) gr-qc/0407014.

[24] V. Iyer and R. M. Wald, "Some properties of Noether charge and a proposal for dynamical black hole entropy," Phys. Rev. D 50, 846 (1994) [gr-qc/9403028].

[25] V. Iyer and R. M. Wald, "A Comparison of Noether charge and Euclidean methods for computing the entropy of stationary black holes," Phys. Rev. D 52, 4430 (1995) gr-qc/9503052.

[26] M. Mars, "Stability of MOTS in totally geodesic null horizons," Class. Quant. Grav. 29 (2012) 145019 arXiv:1205.1724 [gr-qc]].

[27] V. Moncrief and J. Isenberg, "Symmetries of Higher Dimensional Black Holes," Class. Quant. Grav. 25, 195015 (2008) arXiv:0805.1451 [gr-qc]].

[28] C. B. Morrey: Multiple integrals in the calculus of variation, Springer Verlag, Berlin, Heidelberg, New York, 1966.

[29] R. C. Myers and M. J. Perry, "Black Holes in Higher Dimensional Space-Times," Annals Phys. 172, 304 (1986).

[30] I. Racz, "A Simple proof of the recent generalisations of Hawking's black hole topology theorem," Class. Quant. Grav. 25 (2008) 162001 [arXiv:0806.4373 [gr-qc]].

[31] H. S. Reall, "Classical and thermodynamic stability of black branes," Phys. Rev. D 64, 044005 (2001) |hep-th/0104071].

[32] T. Regge and C. Teitelboim, "Role of surface integrals in the Hamiltonian formulation of general relativity," Ann. Phys. 88 286-318 (1974).

[33] T. Regge and J. A. Wheeler, "Stability of a Schwarzschild singularity," Phys. Rev. 108, 1063 (1957).

[34] M. Schechter, "General boundary value problems for elliptic partial differential equations", Commun. Pure and App. Math., Vol XII, 457-486 (1959) 
[35] M. D. Seifert and R. M. Wald, "A General variational principle for spherically symmetric perturbations in diffeomorphism covariant theories," Phys. Rev. D 75, 084029 (2007) [gr-qc/0612121].

[36] R. D. Sorkin and M. Varadarajan, "Energy extremality in the presence of a black hole," Class. Quant. Grav. 13, 1949 (1996) [gr-qc/9510031].

[37] K. Tanabe, S. Kinoshita and T. Shiromizu, "Asymptotic flatness at null infinity in arbitrary dimensions," Phys. Rev. D 84, 044055 (2011) [arXiv:1104.0303 [gr-qc]].

[38] S. A. Teukolsky, "Rotating Black Holes: Separable Wave Equations for Gravitational and Electromagnetic Perturbations," Phys. Rev. Lett. 291114 (1972)

[39] R. M. Wald and A. Zoupas, "A General definition of 'conserved quantities' in general relativity and other theories of gravity," Phys. Rev. D 61, 084027 (2000) |gr-qc/9911095|.

[40] F. J. Zerilli, "Effective potential for even parity Regge-Wheeler gravitational perturbation equations," Phys. Rev. Lett. 24, 737 (1970). 